\title{
Research Square \\ Construction of Structural Geological Model using Monte Carlo Simulation
}

\section{Bruna Catarino Xavier ( $\nabla$ brunacatarino@gmail.com )}

University of Sao Paulo: Universidade de Sao Paulo https://orcid.org/0000-0001-8303-9151

\section{Marcos Egydio-Silva}

University of Sao Paulo

Robert Sadowski

University of Sao Paulo

\section{Bruno Silva}

University of Sao Paulo

\section{Victor Takara}

University of Sao Paulo

\section{Research Article}

Keywords: Structural Geology, Applied Geology, Monte Carlo Simulation Method, Geotechnics

Posted Date: June 7th, 2021

DOI: https://doi.org/10.21203/rs.3.rs-212576/v1

License: (c) (1) This work is licensed under a Creative Commons Attribution 4.0 International License. Read Full License

Version of Record: A version of this preprint was published at Geotechnical and Geological Engineering on August 25th, 2021. See the published version at https://doi.org/10.1007/s10706-021-01967-w. 


\section{Construction of Structural Geological Model using Monte Carlo Simulation}

Bruna Catarino Xavier ${ }^{1}$, Marcos Egydio-Silva ${ }^{1}$, Georg Robert Sadowski ${ }^{1}$, Bruno de Assis Silva ${ }^{2}$, Victor Junji Takara ${ }^{2}$

${ }^{1}$ Institute of Geoscience - University of São Paulo, São Paulo, Brazil

${ }^{2}$ Institute of Mathematics and Statistics - University of São Paulo, São Paulo, Brazil

\section{Abstract}

To optimize the prediction of structural geological conditions in the underground as of data collected at the surface, due to the usual great uncertainties involved, we discuss new perspectives for the construction of structural geological models, bearing in mind the common doubts involved and their implications in the safety of infrastructure works, mining, etc. This paper presents a statistical simulation applied to structural geological measures (dip-dip direction) obtained from schists during the design and construction of civil works through a correlation between surface data with different depth levels. Angular structural geological measures of joints and foliations converted in direction cosines were subjected to the PERMANOVA test to verify the amplitude of differences at different depth levels. The asymptotic results allowed to determine regions of confidence built around centroids through statistical simulation, allowable consistency was considered in regions where the differences in the simulated values were small enough from a practical point of view, taking into account that the difference between joint structures and foliation structures is smaller in the former. The foliation is a characteristic structure of rock deformation just like the joints.

Keywords: Structural Geology, Applied Geology, Monte Carlo Simulation Method, Geotechnics

\section{Introduction}

Seeking to understand the interaction between human activity and geological environment, aiming at positive or negative previsions in a project, and it is need of eventual prevention is the main focus of most of Geology Applied to Engineering, be it civil construction, mining, oil or environment (Hasui \& Mioto 1992, Pastore et al 1998, Sadowski, 2014, among others). The correct prediction of structural geology involving the spatial dispositions of contacts between different lithologies and their respective discontinuities are one of the main geological determinants of the stability of large foundations and excavations, open-cast or underground. All stability analyzes carried out in rock engineering are subject to uncertainties thus generating geological risks, these risks may cause the execution of the work not to meet the preestablished requirements, causing financial losses and risks to human life, as well as serious problems of environmental issues.

Geological risk assessment in places of difficult viewing and access, such as underground conditions, aims to minimize the inherent faults in the data collection or exploration of the conditions of the rock masses in-depth, reducing errors is important to the safety of employees during the work, the control of the planned expenses, as well as the durability of the civil works, promoting social, financial and environmental safety. The mistake in the predictability is related to the number of uncertainties that are contained in several stages of the works, which are inseparable in geotechnics (Costa, 2005), because of this, Ang \& Tang (2007) highlight the importance of identifying them.

In general, geological risk analyses are performed through deterministic analysis of the geotechnical parameters. These methods frequently do not quantitatively consider the randomness and variability of the parameters involved in the project, making it difficult to identity uncertainties (Hoek et al, 1997). In this case, the analysis does not demonstrate exactly the degree of predictability of the underground conditions of the works, in other words, the identification of the error percentage in the geological model. In the last years, there has been a great interest in incorporating methodologies that 
allow estimating the uncertainty associated with geotechnical studies, as is the case with probabilistic methods or statistical simulation (Ge et al., (2011); Montoya \& Assis (2011b); Hudson and Feng, (2015)).

The main proposal of the present study is to use applied statistical analyses, to structural geological data, aiming to optimize the predictions of the geological conditions in depth. The specific goals involve the following topics: i) compilation of geological and structural data of the study area, ii) transformation of directional data into linear data for descriptive statistical analysis, iii) correlation between surface data and depth data, iv) construction of the structural geological models using statistical simulation with the use of the Monte Carlo technique.

This study is applied in a practical example in order to try to answer the question: "Is it possible to know the data in depth using simulation?" In the search for an appropriate answer to this question, we use the Monte Carlo simulation, to better understand the correlation between the data collected in the field, on the surface, with those existing in-depth, which is difficult to predict and/or access.

\section{Geological Setting}

The structural geological data were obtained during the construction of a hydroelectric dam, in the Southeast of Brazil, in the Minas Gerais state (Fig. 1a). This area is composed of complex geology, the main tectonic unit is simplified in figure $1 \mathrm{~b}$ with identification of the collection site. The topography of this area is smooth, being dissected by several streams along the Araguari river valley, with basement outcrops belong to the Brasilia belt (Fig.1b), this unit is composed predominantly of metasediments rocks of the Araxá Group, local granitoid rocks and gneiss from the Maratá Complex.

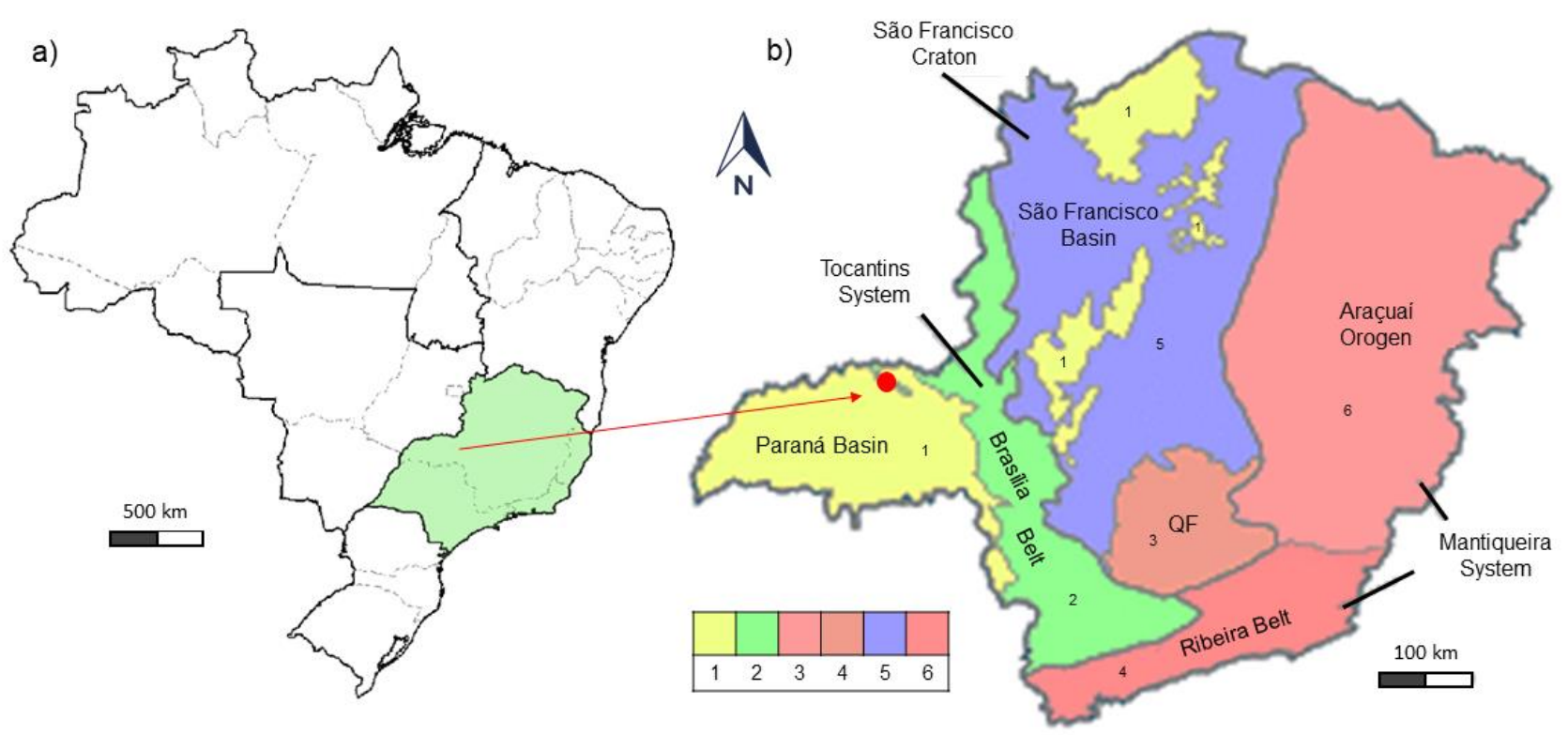

Fig. 1 a) Boundary map of Brazilian Regions with a green highlight on the Southeast Region b) Minas Gerais State map shows the compartmentalization of geotectonic units identified by the number (1) Paraná Basin; (2) Brasilia belt; (3) Quadrilátero Ferrífero unit (4) Ribeira belt (5) São Francisco Craton and São Francisco Basin and (6) Araçuaí belt, and the red dot identifying the data collection location

The dam is situated in the hydrographic basin of Araguari river (Fig.2b) in the realm of schists belonging to the Araxá Group, strongly foliated, with tectonic contacts (shear zones) with the local gneisses, causing parallelism in both gneissic banding and granitic foliation (Pacheco et al., 2017). The Maratá Complex represents a narrow strip of granitoid rocks interlayered with the Araxá metasediments and is composed of augen-gneiss and porphyritic meta-granite with quartz, potassium feldspar, biotite and muscovite, frequently carrying a mylonitic texture (Pimentel, 2016; Pacheco et al., 2017) 
(Fig.2c). Amphibolites interspersed in the metasediments of the Araxá Schists were interpreted as remnants of a maficultramafic magmatism (Strieder \& Nilson, 1992; Pimentel et al., 1999); additionally, there are quartzite and micaceous mica schists, included calcschists, chlorite-moscovite schist, garnet biotite schist, staurolite schist and feldspar schists, with few paragneiss and marble intercalations. (Pimentel, 2016). The presence of anastomosed foliation, intra-folial folds, three generations of metamorphic foliations, stretching, mineral lineations, and all these structures denote the complex tectonic evolution of these metasediments (Valeriano, 1993).
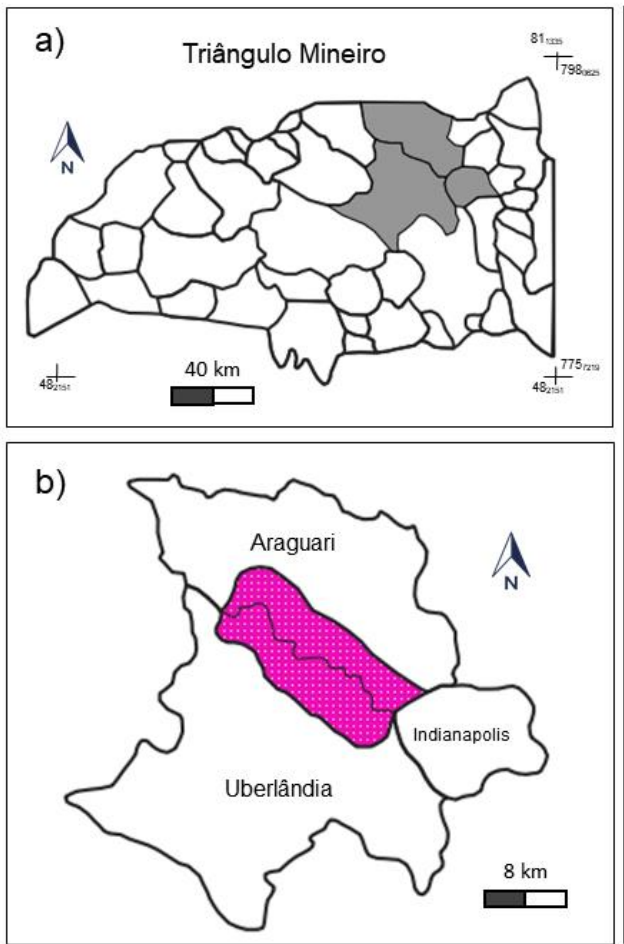

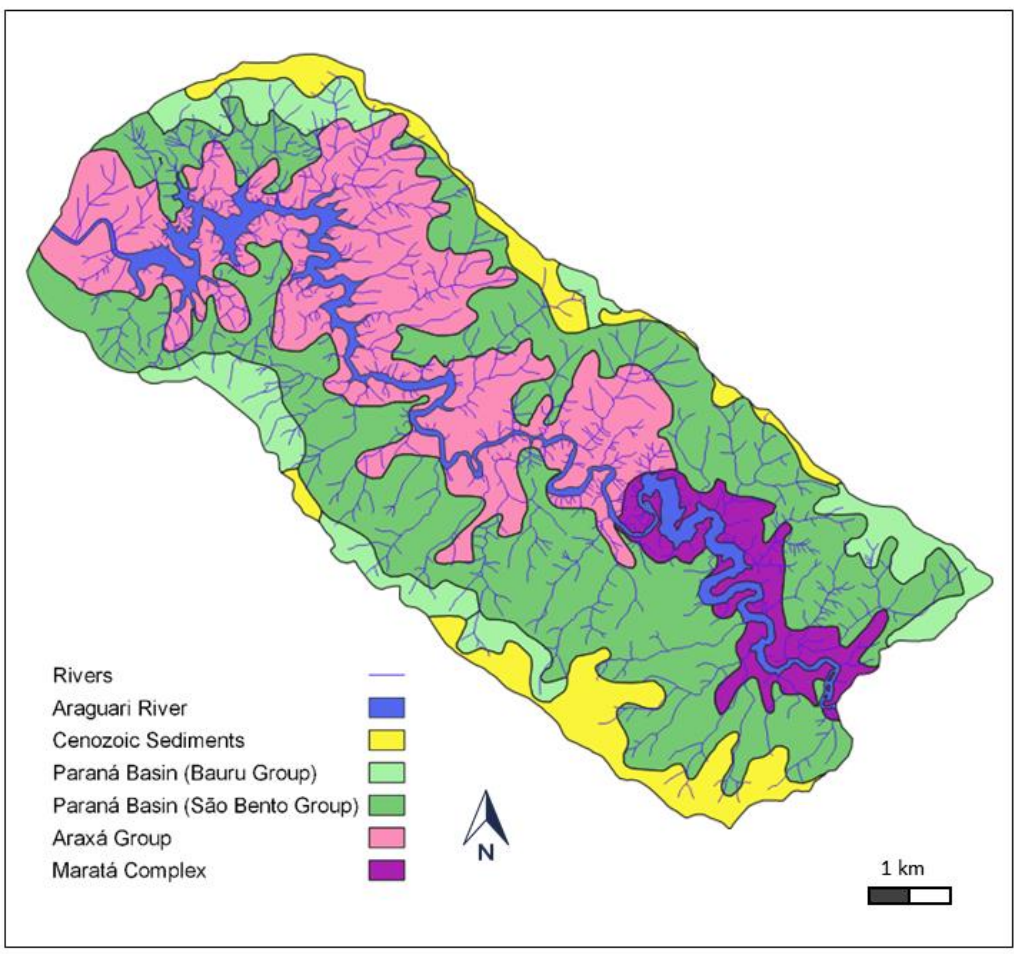

Fig. 2 a) Map of the municipalities of the southwest of the Minas Gerais State. In gray the municipalities of Araguari, Uberlandia and Indianapolis b) Localization of the hydrographic basin of the Araguari river c) Geological map restricted to the region of the Araguari river basin with the main units in this area. Schist of the Araxá Group (pink color) and gneiss of Maratá Complex outcrop along the riversides

Araxá schists was formed by the metamorphism of sedimentary rocks that occurred during the compressive phase of the Neoproterozoic orogeny (PanAfrican/Brasilian), of which, two phases of progressive deformation can be individualized in a simple shear regime. The first deformation phase is responsible for the development of the regional foliation with $\mathrm{N}$ $\mathrm{S}$ direction dipping approximately $50^{\circ}$ to west, this foliation is the axial plane of isoclinal folds. The second deformation phase corresponds to crenulation formed by small folds whose axial planes are oriented preferably NW-SE/sub-vertical. This structure deforms the regional foliation of the N-S direction with plunge to east, and this change in the dip direction could be responsible for the dispersion of the foliation diagram (Pacheco et al, 2017).

\section{Descriptive Analyses of Structural Geological Data}

A sampling of the structural geological data was performed in two different ways: i) first a set of the measurement were obtained during the surface mapping, resulting in 396 measures, and ii) during the construction, 953 new additional data were gathered in the built slope, the headrace tunnel and appurtenant dam structures (Fig. 3). 

All measured data are in a dip and dip-direction notation, divided between two different structures, foliation and joints. The foliation refers to repetitive and different compositional layering in the metamorphic rocks and, the joints are discontinuities formed as a result of regional stress after the rock consolidation.

a)

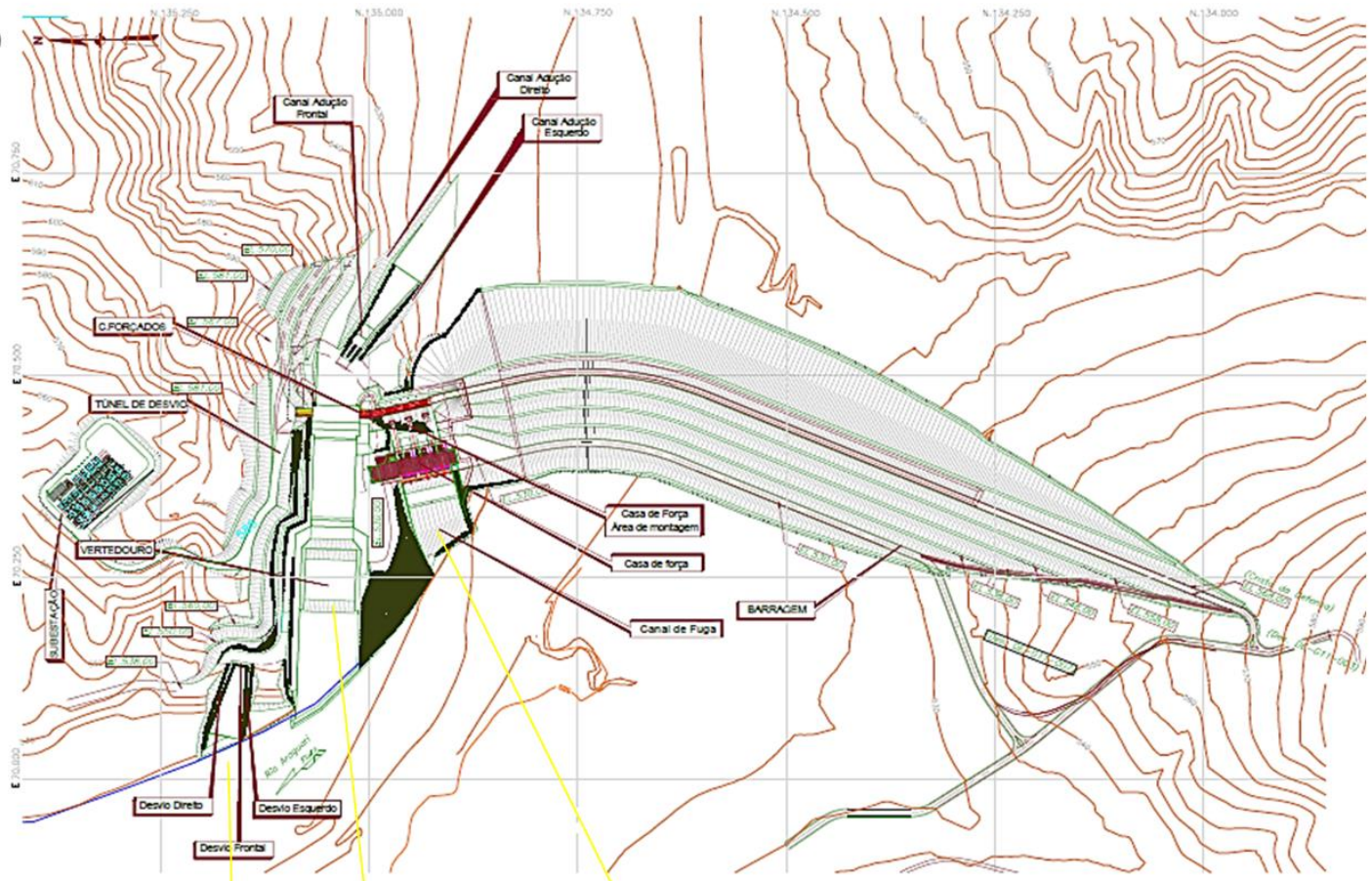

b)

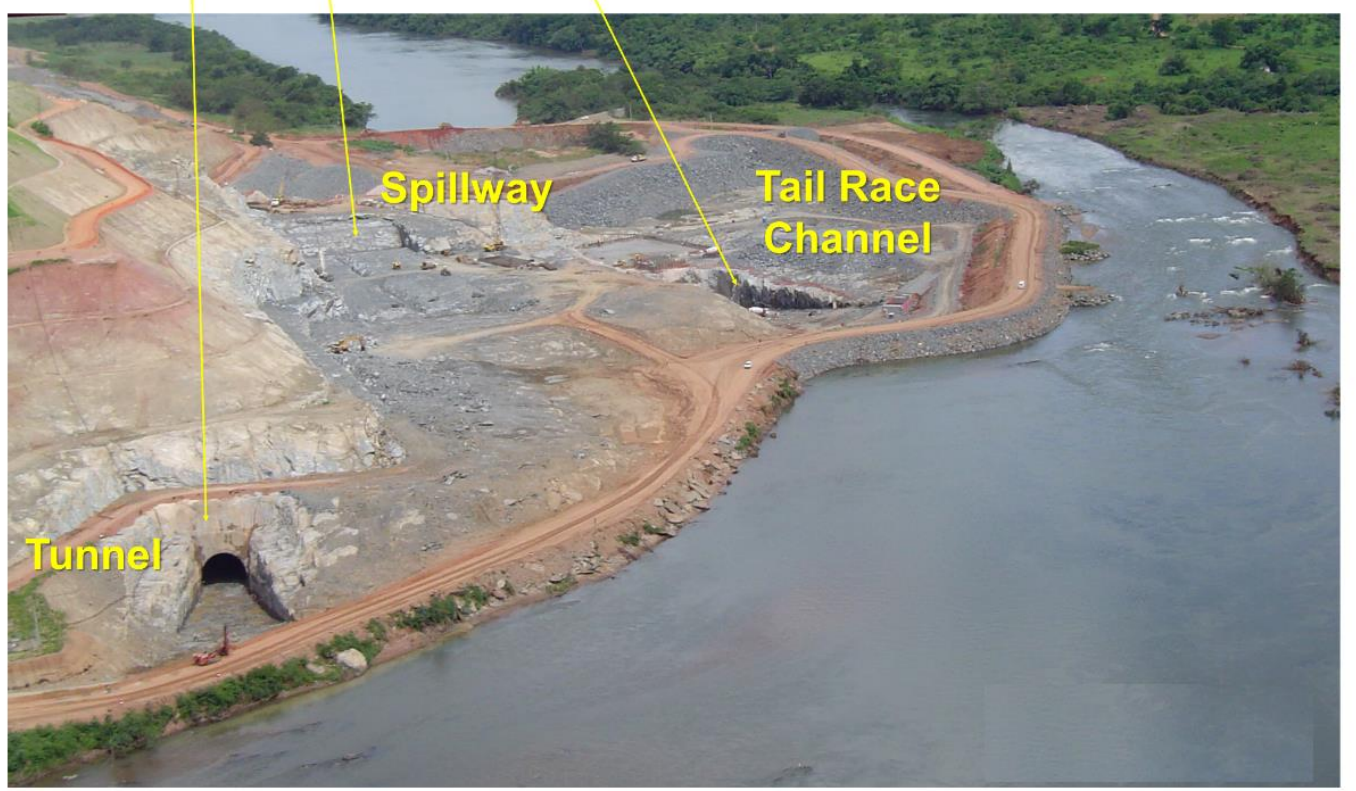

Fig. 3 a) Illustration of the dam structure in the topographic map b) Photo with the identification of data sampling sites

In order to organize the statistical analyses, the structural data were separated into their different types, place of measurement, surface and depth intervals (Tab.1). All data are present in supplementary materials 1, which contains the level where the measure was taken, type of structure (foliation and joints), and the transformation between dip dipdirection to direction cosines. 


\begin{tabular}{|c|c|c|c|c|}
\hline Elevation & Depth & $\begin{array}{c}\text { Number of } \\
\text { measures }\end{array}$ & Joints & Foliations \\
\hline surface & $0 \mathrm{~m}$ & 396 & 233 & 163 \\
\hline $558-540$ & $0-18 \mathrm{~m}$ & 61 & 39 & 22 \\
\hline $540-530$ & $18-30 \mathrm{~m}$ & 115 & 79 & 36 \\
\hline $530-520$ & $30-40 \mathrm{~m}$ & 177 & 197 & 32 \\
\hline tunnel & $50 \mathrm{~m}$ & 314 & 211 & 75 \\
\hline $520-500$ & $50-60 \mathrm{~m}$ & 286 & & 117 \\
\hline
\end{tabular}

To perform the statistical analysis, both at the surface and depth, all data were converted into direction cosines. The transformation of dip and dip-direction measures into direction cosines $\mathrm{X}, \mathrm{Y}, \mathrm{Z}$ were made using the usual spherical coordinate systems (Mardia, 1972 and Fischer et al., 1993) with lower hemisphere (Fig.4). This type of transformation is important to enable the simulation of the statistical model. The y position corresponds north direction, the dip value is transformed into $\theta$ by equation (3.1) and dip-direction value into $\phi$ by means of the equation (3.2)

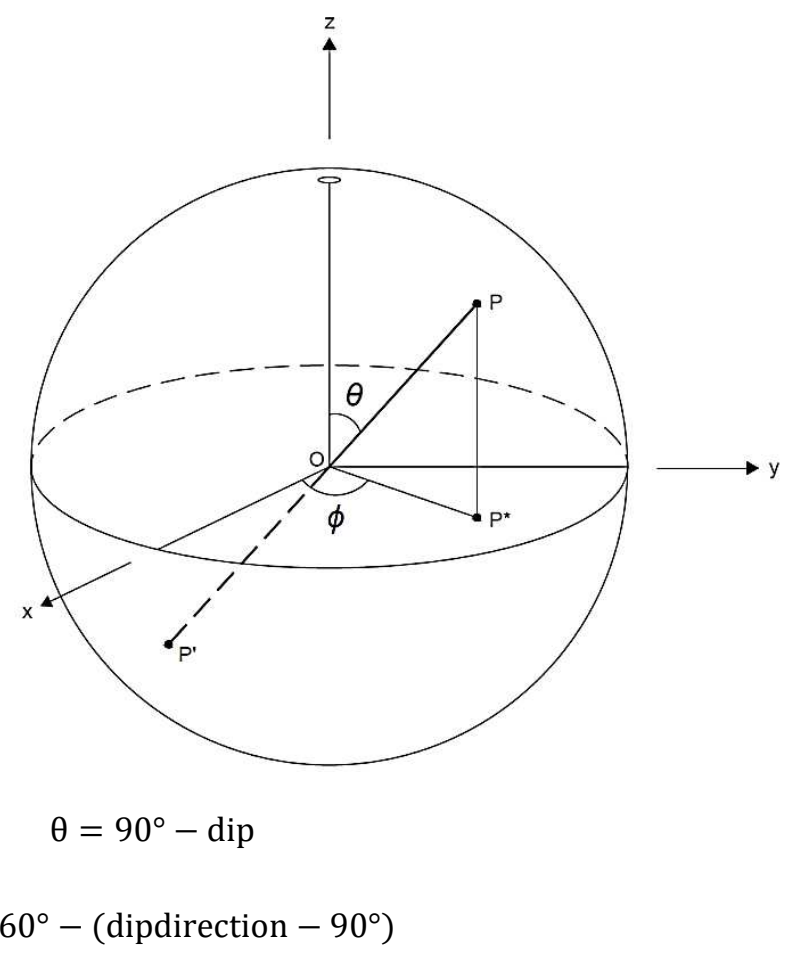

$\phi=360^{\circ}-\left(\right.$ dipdirection $\left.-90^{\circ}\right)$

Fig. 4 Ilustration of the position coordinates used to calculate the direction cosines, the $\theta=$ latitude and $\phi=$ longitude, $P$ represent polar projetion of data (Fisher et al 1993).

\subsection{Foliation and Joints}

After the transformation of the measurements, it is possible to observe the spatial distribution of all the data dip-dipdirection that were transformed in direction cosines (Fig.5a), where the red dots represent the direction cosines data that were obtained in-depth, the blue dots show the direction cosines data collected in the surface, each point represent one measure. The representation using stereographic projection of all these dada that were transformed in direction cosines, including foliation and joints planes, shows the spatial distribution of all the planes (Fig. 5b), however, it is not possible to distinguish between foliation and joints. To identify the statistical significance of each planar structure, it is necessary to proceed with an analysis separately. We begin analyzing the direction cosines of the foliation and joints differently, 
and it allows to visualize the direction cosines obtained for the foliation measures, it is noted that there is a greater homogeneity in the spatial distribution, both in surface and in-depth (Fig.5c), which can be seen by the representation through stereographic projection that shows a single main concentration, with dip-dip-direction 49/277 (Fig.5d).

The representation of direction cosines for joint measures exhibit two principal concentrations, the red dots, representing depth joints, which have a higher frequency than blue dots, expressing surface joints (Fig.5e). The projection of the 904 joint data, highlights four different joint systems, the most significant concentration named J1, presents the dip-dipdirection 87/251. The $J 2$ set (52/281) has characteristic the parallelism (sub parallelism) with the foliation planes. Two other sets, statistically less important, $J 3(85 / 344)$ and $J 4(30 / 120)$ complete the four joint systems identified in the area (Fig.5f). The correspondence between the representation of data and main planes, cannot be distinguished into projection diagrams.

a)

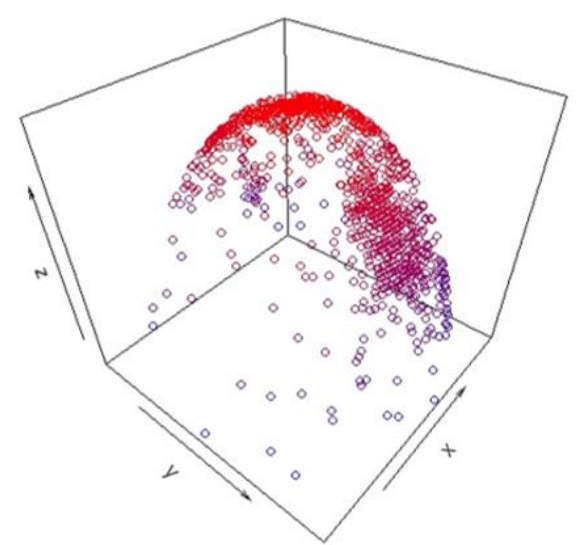

c)

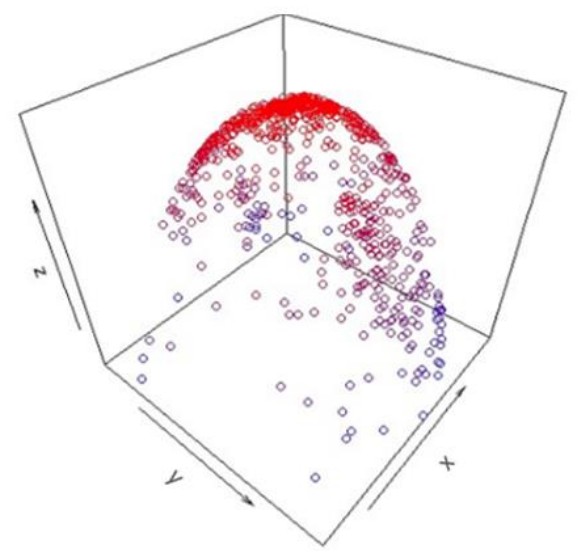

e)

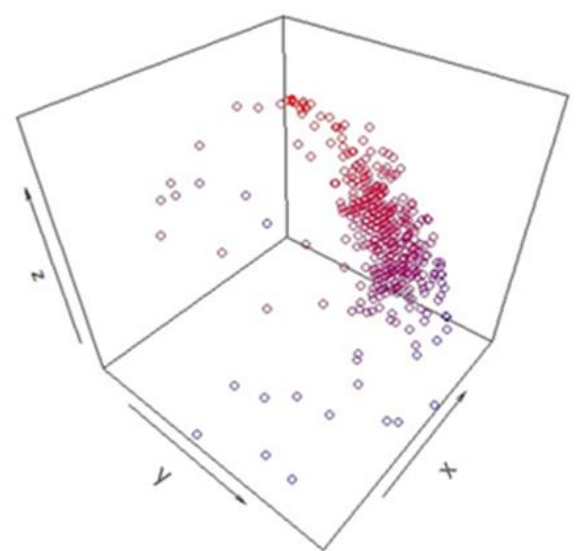

b)

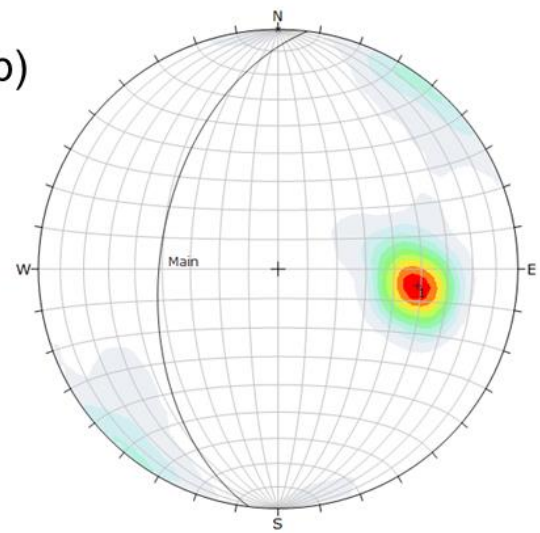

d)
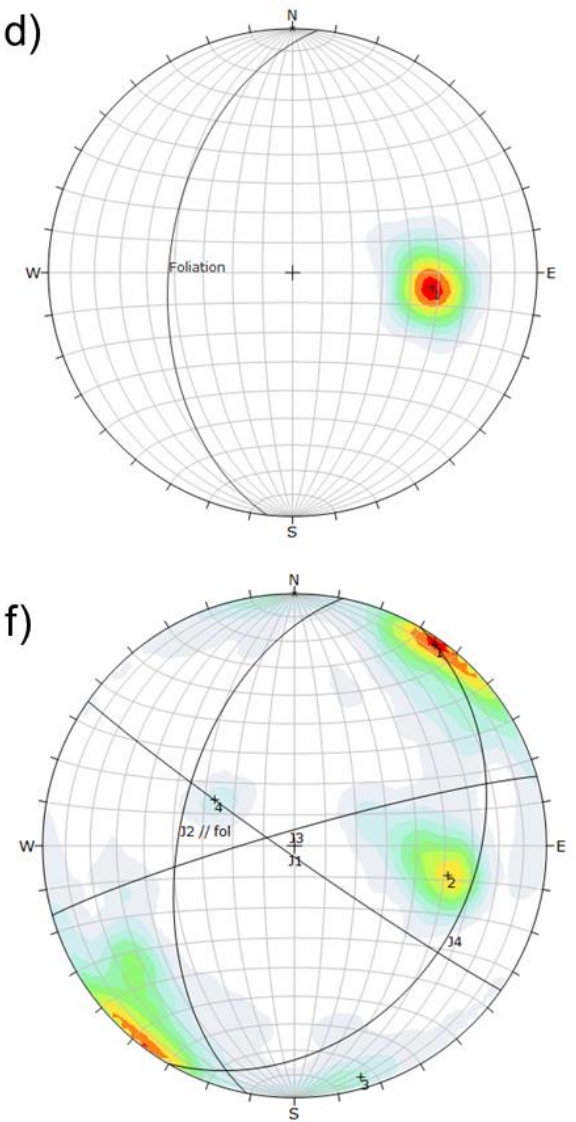

Fig. 5 Correlation between direction cosines and stereographic projections in different situations a) The projection of direction cosines of all the 1349 measurements (foliation and joint at surface and depth), where the red and blue 
related to foliation (445 measurements) d) Using stereographic projection the mean value of the foliation is $48 / 276$ (dip and dip-direction) e) Direction cosines diagram using 904 data of joints f) Stereographic projection of the joints highlights four distinct sets: J1 (88/215), J2 (54/282), J3 (85/344) and J4 (30/120) using dip dip-direction notation

For the correlation between surface - depth data and to achieve a 3D structural model, it is necessary to evaluate the statistical distribution of the foliation and joints for different depth levels. The analysis, for joint and foliation planes, was performed in three different depth intervals, as follows: we highlight the projections of direction cosines from the surface to 15 meter of depth (Fig.6a), then between 15 and 45 meters deep (Fig. 6b), and finally the stretch between 45 and 60 meters (Fig.6c). The respective representations of stereographic projections, in each level, are illustrated in figures 6d, 6e and 6f, which were evaluated separately, show a good similarity between the three distinct depths, with a principal direction average N-S dipping to West. The larger quantity of measurements in the last stretch was due to the greater amount of fractures present in the tunnel.
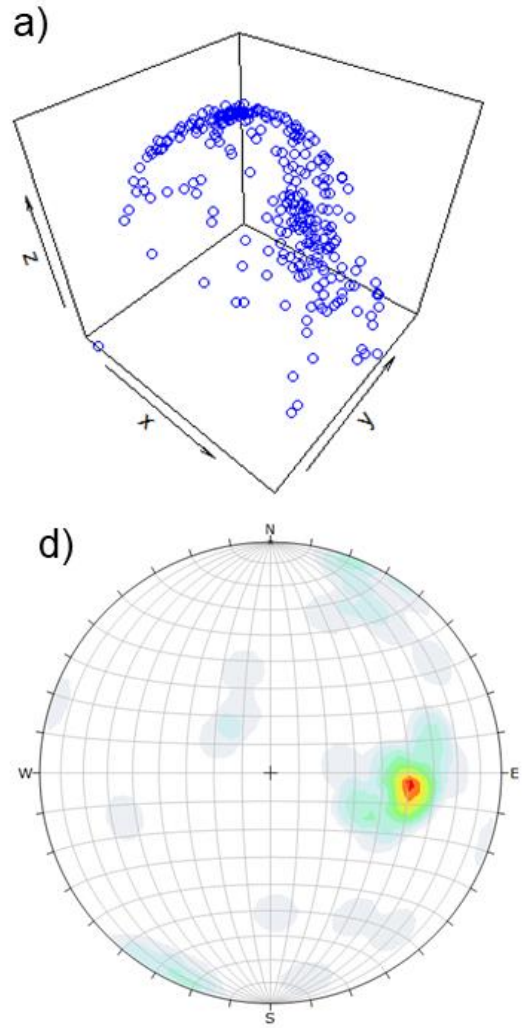

b)

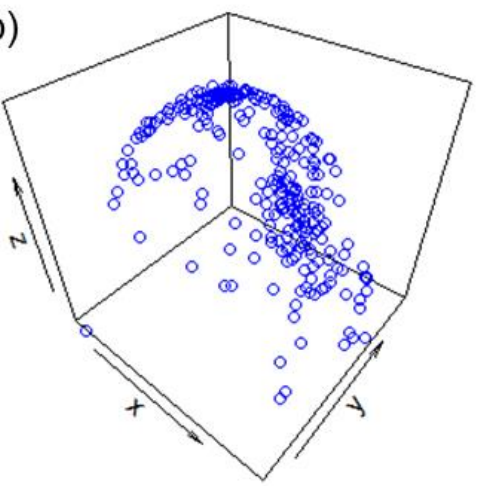

e)

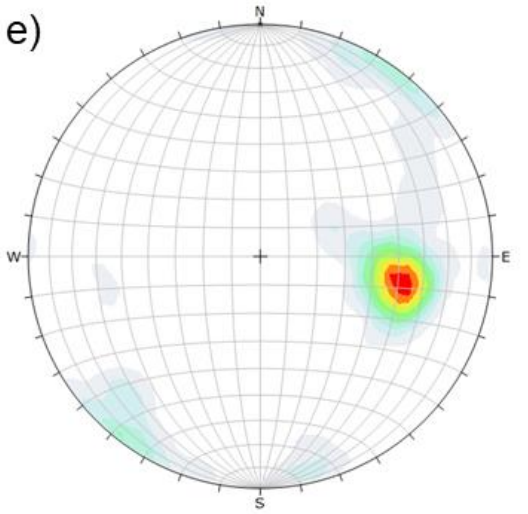

c)

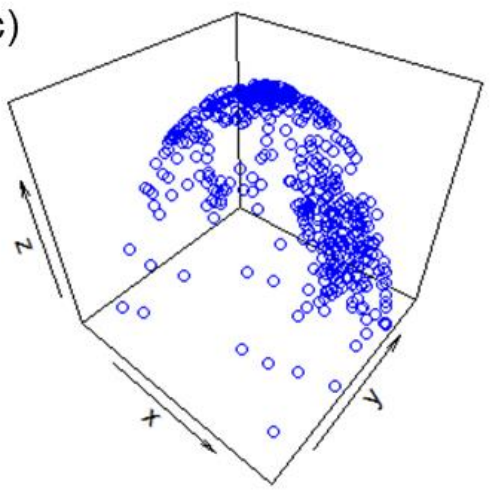

f)

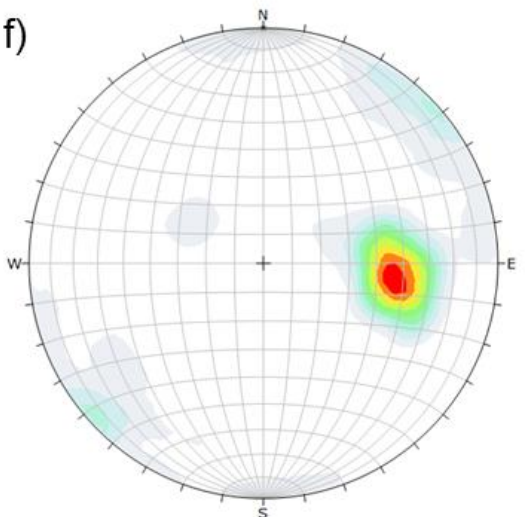

Fig. 6 Spatial representation of direction cosines and stereographic projection of the foliation and joints orientations at the three different levels of depth a) The direction cosines graphic for the measure of the first level of $\mathbf{0}$ to 15 meter of depth, with $\mathbf{4 3 1}$ measures b) The direction cosines for measures of the second level of 15 to 45 meter of depth, with 319 measures c) The direction cosines for measures of the third level of 45 to 60 meter of depth, with 599 measures. The stereographic projections of the three different levels are very similar (d, e and f). The red point in each diagram represents the main concentration of the measurements $(\sim \mathrm{NS} / 50 \mathrm{~W})$ with some scattered spots in gray

\subsubsection{Foliation Data}

The foliation represents the main structure in this area it direction is almost N-S with plunge $50^{\circ}$ to West or approximately 50/270 in the dip/dip-direction notation (Fig.7). The stereographic projection of foliation measurements reveals the similarity in the two depth ranges, from 0 to 30 meters, was collected 90 measures, and between 31 to 60 meters, was 
a)

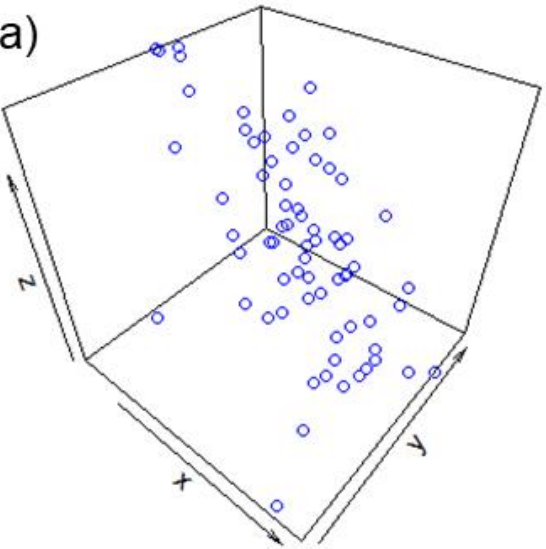

c)

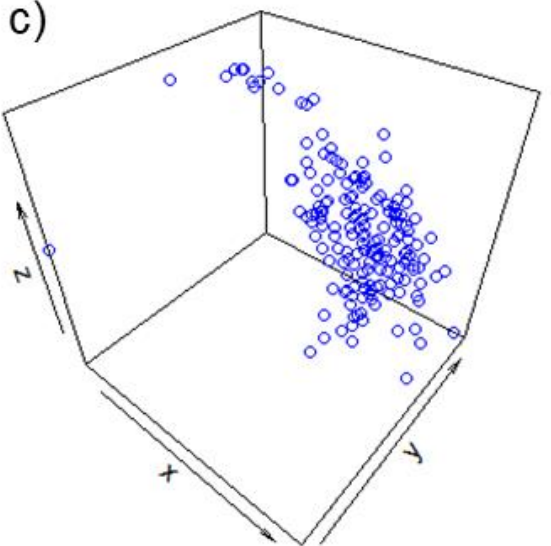

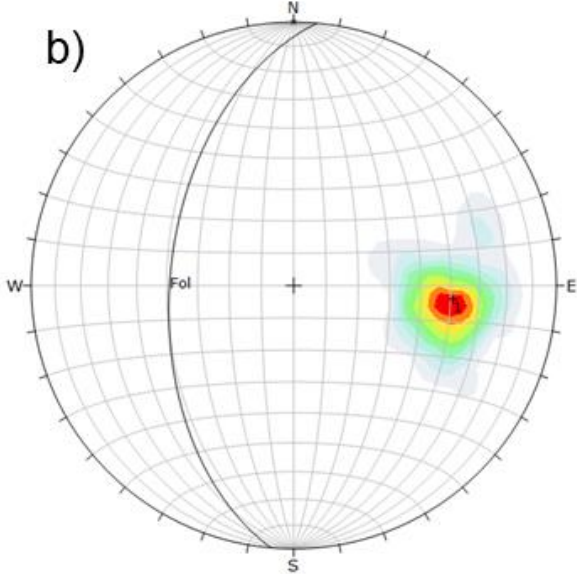

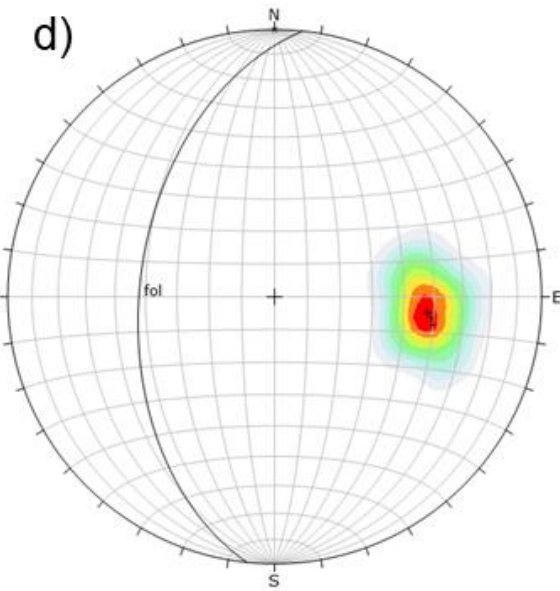

Fig. 7 The direction cosines and the stereographic projections for foliation measurements obtained from 0 to 40 meters of depth, respectively diagrams (a) and (b), with 90 measures. Statistically, the average value is 50/276. Diagrams c and d correspond to the same representations for the 41 to 60 meters of depth, with 192 measures. At both depth intervals, the mean value corresponds to the spatial orientation 49/276 (dip dip-direction notation)

\subsubsection{Joint Data}

Analyzing the joints measurements obtained for the stretch of 0 to 40 meters deep, it is possible to identify the $\mathrm{J} 1$ joint system as being the most statistically significant, with dip dip-direction 88/037 and, the J2 joint system, whose spatial distribution of it is direction cosines (Fig.8a) and stereographic projection (Fig.8b) show a sub-parallelism with the regional foliation J2//fol (53/283). Two other systems (J3 and J4) can observed in the diagram, is quantitatively less expressive, oriented, respectively to 86/175 and 56/077 (dip-dip direction notation) (Fig.8b), at this level there are 263 measures.

When it is observed the 41 to 60 meters level (Fig. 8c and 8d), is evident a new concentration named J4 that is opposite $\mathrm{J} 2$, and there are 211 measures at this level. If isolating the measurement obtained in the tunnel, there is a different configuration, it is possible to identify the only $\mathrm{J} 1$ in the direction cosine graphic (Fig. 8e), in the stereographic projection (Fig. 8f) there are all the joint direction systems with low concertation. 

data (Fig.9).

a)
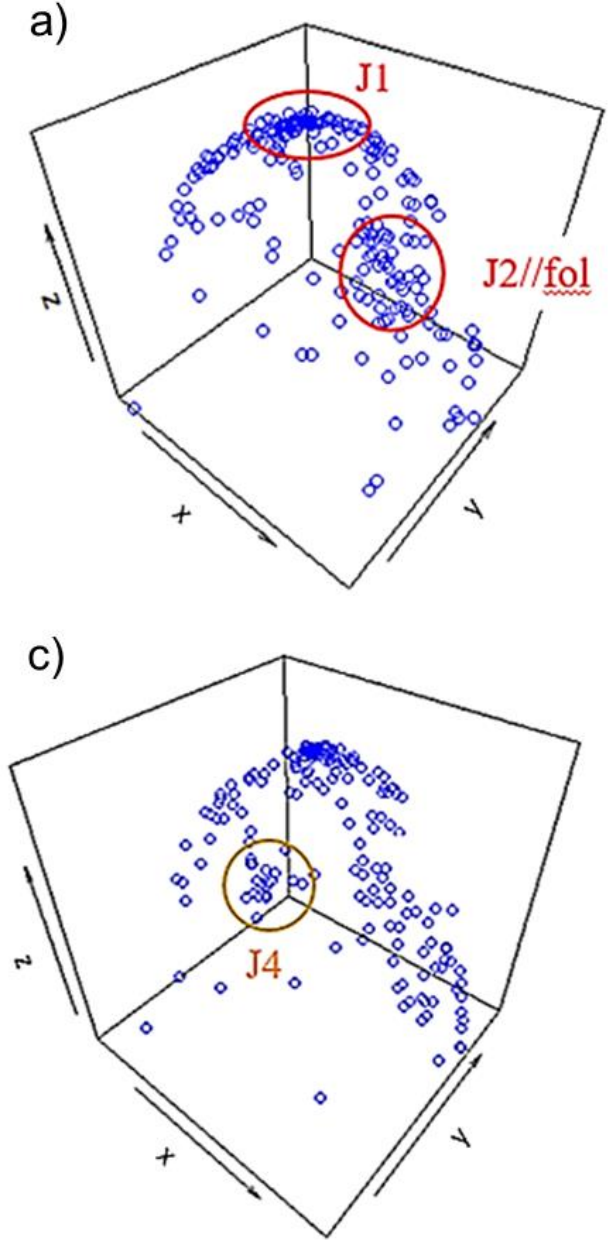

e)

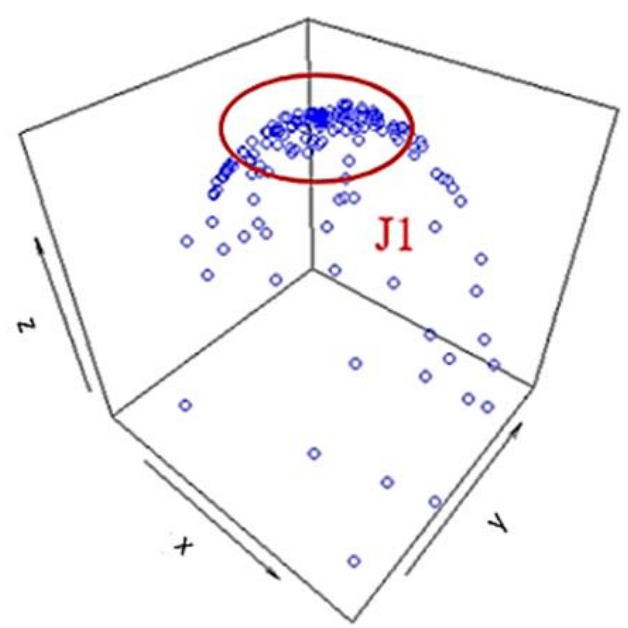

b)

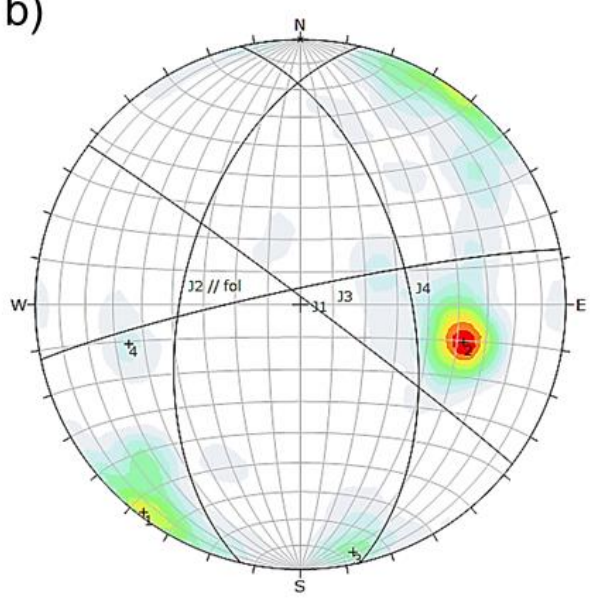

d)

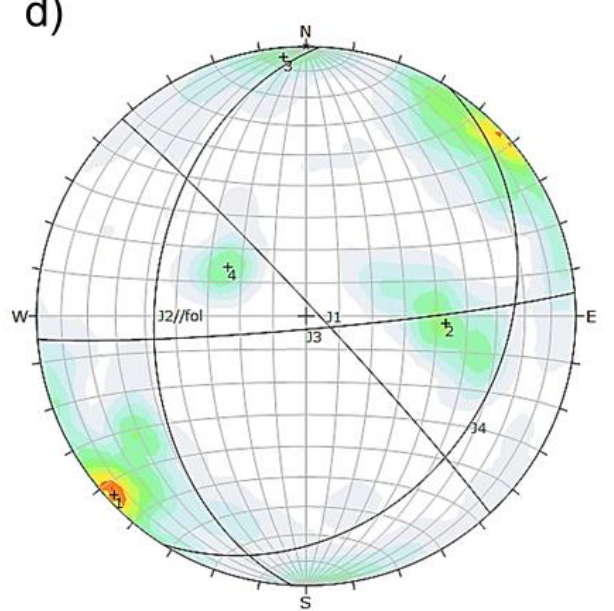

f)

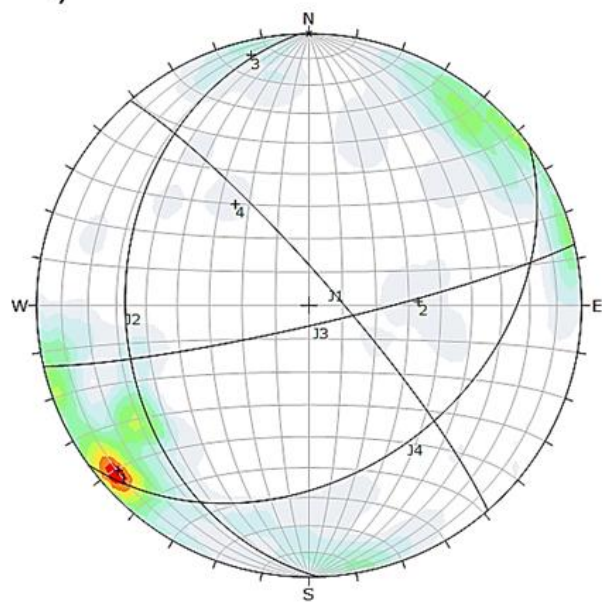

179 Fig. 8 Projections of the direction cosines regarding the measurements of joints obtained at the following depths: 180 (a) 0 to 40 meters, showing the $\mathrm{J1}$ and $\mathrm{J} 2$ system concentration; (c) 41 to 60 meters, highlighting the $\mathrm{J4}$ concentration; (e) and 50 meters-depth in adduction tunnel, with a highlighting of $\mathrm{J} 1$ system. The respective stereographic projection diagrams for theses depths show the following joint systems: (b) J1 (88/037), J2//fol (53/283), J3 (85/355) and J4 (56/077); (d) J1 (87/047), J2//fol (43/273), J3 (85/175) and J4 (28/122) and (f) J1 


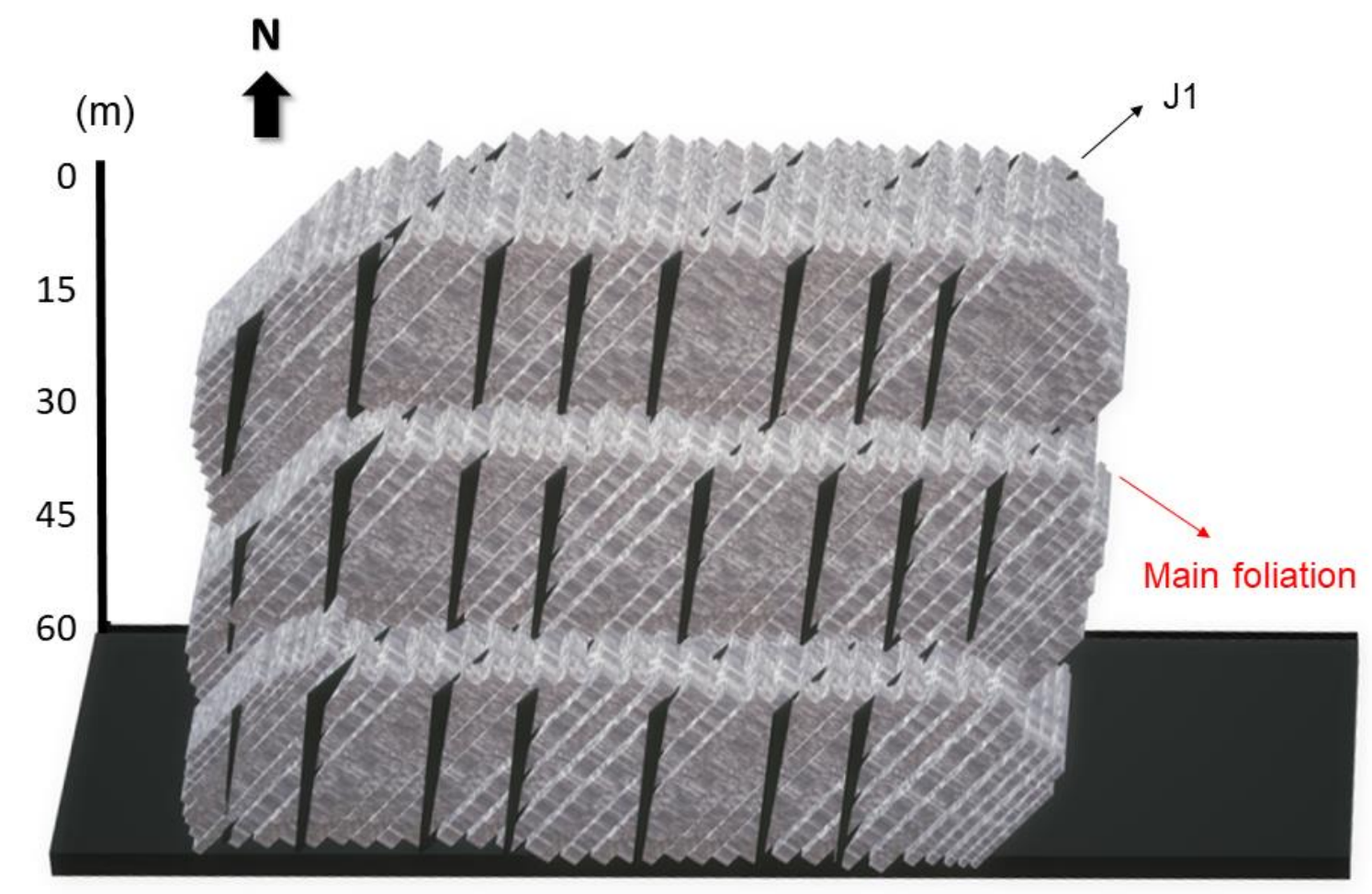

Fig. 9 Simplified block diagram showing the foliation, in the gray color, and the $J 1$ joint system in black color

\section{Monte Carlo Simulation}

After the descriptive analyses of structural geological data, it is important to understand the statistical distribution of data, since the Monte Carlo simulation consists of generating random numbers from of the real data with a known distribution. To analyze the data distribution, some techniques were used, initially, it was applied multivariate analysis of variance (MANOVA) model (Johnson and Wicher, 2007) for the direction cosines, considering the type of structure (foliation and joint) and level of depth as explanatory variables. However, the assumption of normality was not met, in others words, the data do not have a normal (Gaussian) distribution, even though homoscedasticity was verified, that in terms of statistics, homoscedasticity occurs when the variance in scores on one variable is somewhat similar at all the values of the other variable, therefore the variance of the error is constant.

Then was applied a permutational multivariate analysis of variance (PERMANOVA), which is a non-parametric model to test the hypothesis that the centroids of the groups are equal (Anderson, 2013), this analysis is used to compare groups of objects and test the null hypothesis that the centroids (confidence region - CR detailed below) and dispersion of the groups as defined by measure space are equivalent for all groups.

It was verified, statistically, the existence of an interaction effect between different depth levels and type of structure, tests of the depth effects were also performed for each structure, being, in both cases, significant with p-value $>0.01$, this verification was done using the statistical significance analysis (equation 4.1)

$$
p=1-\alpha
$$

The p-value is a result of interaction or significance, $\alpha$ is the level of significance, that is, the probability of the study rejecting the null hypothesis, given that the null hypothesis was assumed to be true, when the p-value is below 0.05 , it means that the analysis confidence region has a low error.

Although the results of PERMANOVA indicate that there is a significant difference between the direction cosines of the surface and depth, from a statistical point of view, it does not give us the magnitude of this difference from a real situation, 
which it was understood as an incomplete result for what one wanted to investigate, this happens because the number of measures between surface and depth is different. Since it is known that p-value naturally decreases as the sample size increases (Krueger and Heck, 2019), it was decided to complement the problem from another perspective. In Mardia et al (1995), a version is presented for the central multivariate limit theorem, in which the average vector of a sequence of independent random vectors have a asymptotic multivariate normal distribution, once the sample is large, we then assume that this asymptotic approach is reasonable (Supplementary Material 2).

Using this result, it is possible to estimate a tridimensional parameter $\theta \in \mathbb{R}$ from the direction cosine sample (X, Y, Z) with the observed data we end up with a point estimate, which is the corresponding observed value of $\hat{\theta}$ (simulated data). We know that estimator $\hat{\theta}$ is a random variable (simulated data) and we often prefer to determine a confidence region $(\mathrm{CR})$ for $\theta$. A confidence region is a random subset of $\mathbb{R}$ (determinate by appropriate statistics) such that it is "confident", at a certain given level, that this region contain $\theta$ :

$$
p(\theta \in \mathbb{R})=1-\alpha
$$

Confidence regions are particularly useful when a hypothesis $\mathrm{H}_{0}$ on $\theta$ is rejected, due they eventually help in identifying which components of $\theta$ is responsible for rejection (Härdle and Simar, 2012) (Supplementary Material 3).

The equation 4.3 describe a confidence region for $\mu$ :

$$
C R=\left\{\mu \in \mathbb{R} \mid(\mu-\bar{x})^{\top} S^{-1}(\mu-\bar{x}) \leq \frac{p}{n-p} F_{1-\alpha ; p, n-p}\right\}
$$

The $\mu$ value represent the ellipse of the simulation data. The $\mathrm{CR}$ is the interior of an iso distance ellipsoid in $\mathbb{R}$ centered at $\bar{x}$, with a scaling matrix $S^{-1}$ and a distance constant $\frac{p}{n-p} F_{1-\alpha ; p, n-p}$.

When $\mathrm{p}$ is large, ellipsoids are not easy to handle for practical purposes. Thus, we are interested in finding confidence intervals for $\mu 1, \mu 2 \ldots \mu$ for that simultaneous confidence on all the intervals reaches the desired level (1- $\alpha)$ (Härdle and Simar, 2012). With the described and calculated CR, it is possible to analyze the ellipses behavior obtained in the simulated data (Supplementary Material 4).

The graphic representation of confident ellipse for foliation and joints data shows that the black ellipse is representing the simulated surface data and red ellipse represents the simulated depth data, when the ellipse is larger it means that there is a variability of the data and them is greater in relation to centroid, this happens due quantity of measure. In the case of this example, there are more measures in depth than surface, then the ellipse that represents depth is smaller than surface ellipse in both analyzes graphs (Figs. 10 and 11).

To perform the correlation between surface and depth using the simulated data, the distance between the two ellipses is measured, when the ellipses are overlapping, it means that the simulation data is equal, if there is a distance between them, it means that there is a difference, in general we can obtain the magnitude of this difference with measure of $\mathrm{Z}$ axis. The $\mathrm{Z}$ direction cosine can range from 0 to 1 , where 0 means that the both simulation data is equal, if the distance is 1 , it means that the simulation data for surface is totally different to depth, in others words, if the distance is 0 we will have $0 \%$ difference between surface and depth, in the other hand, if we have the distance 1, we will have $100 \%$ difference between surface and depth simulation data, therefore, if the distance is 0.05 it is means that the difference between surface and depth simulation data is only $5 \%$. 


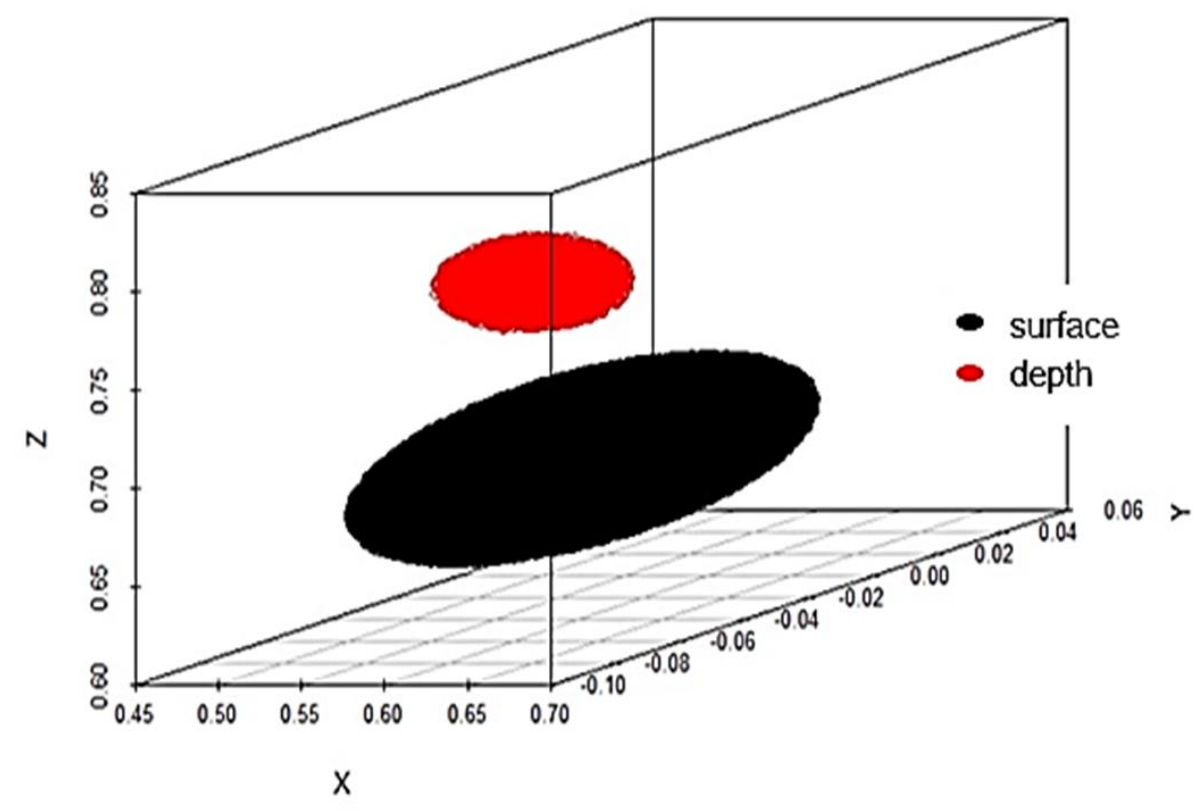

Fig. 10 Graphic representation of confidence ellipse of foliation data, the three axes correspond to direction cosines and red ellipse is a simulate data of depth and the black ellipse is a simulate data of surface

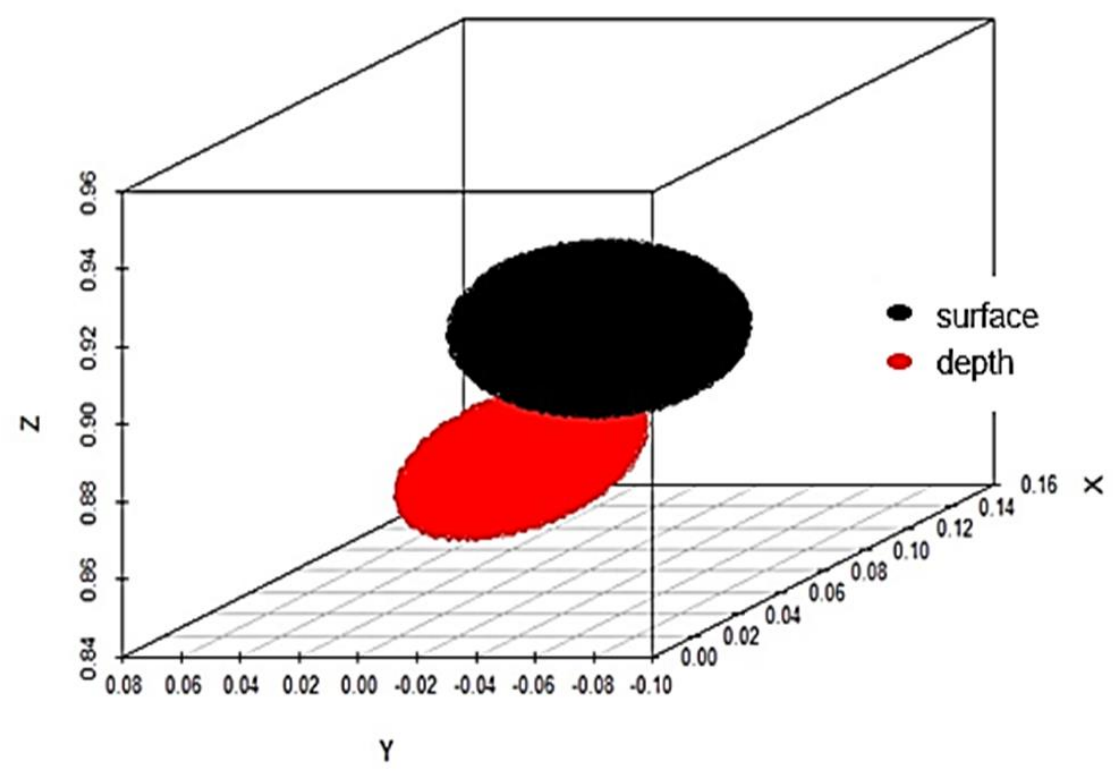

Fig. 11 Graphic representation of confidence ellipse of joints data, the three axes correspond to direction cosines and red ellipse is a simulate data of depth and the black ellipse is a simulate data of surface

The simulation for foliation data presents a difference between surface and depth data. This difference can be observed using the distance between the ellipses, in this case, distance is around 0,05 (direction cosines unit), but in terms of magnitude scale, this difference does not change the feature of foliation measure (Fig. 12). Therefore, there is a resemblance between surface and depth, if it considers that $0,04-0,05$ for direction cosines $\mathrm{Z}$ means that the difference is around $4 \%$ then the simulation is representing the real data for the foliation data. For joint simulation (Fig.13), there is the similarity of the size of ellipse due to less variability in average values, the distance between ellipses, that means a difference with respect of surface and depth data, in magnitude scale, has 0,02-0,04 for direction cosines Z means that the difference is around $4 \%$, thus it has the same situation for foliation. 

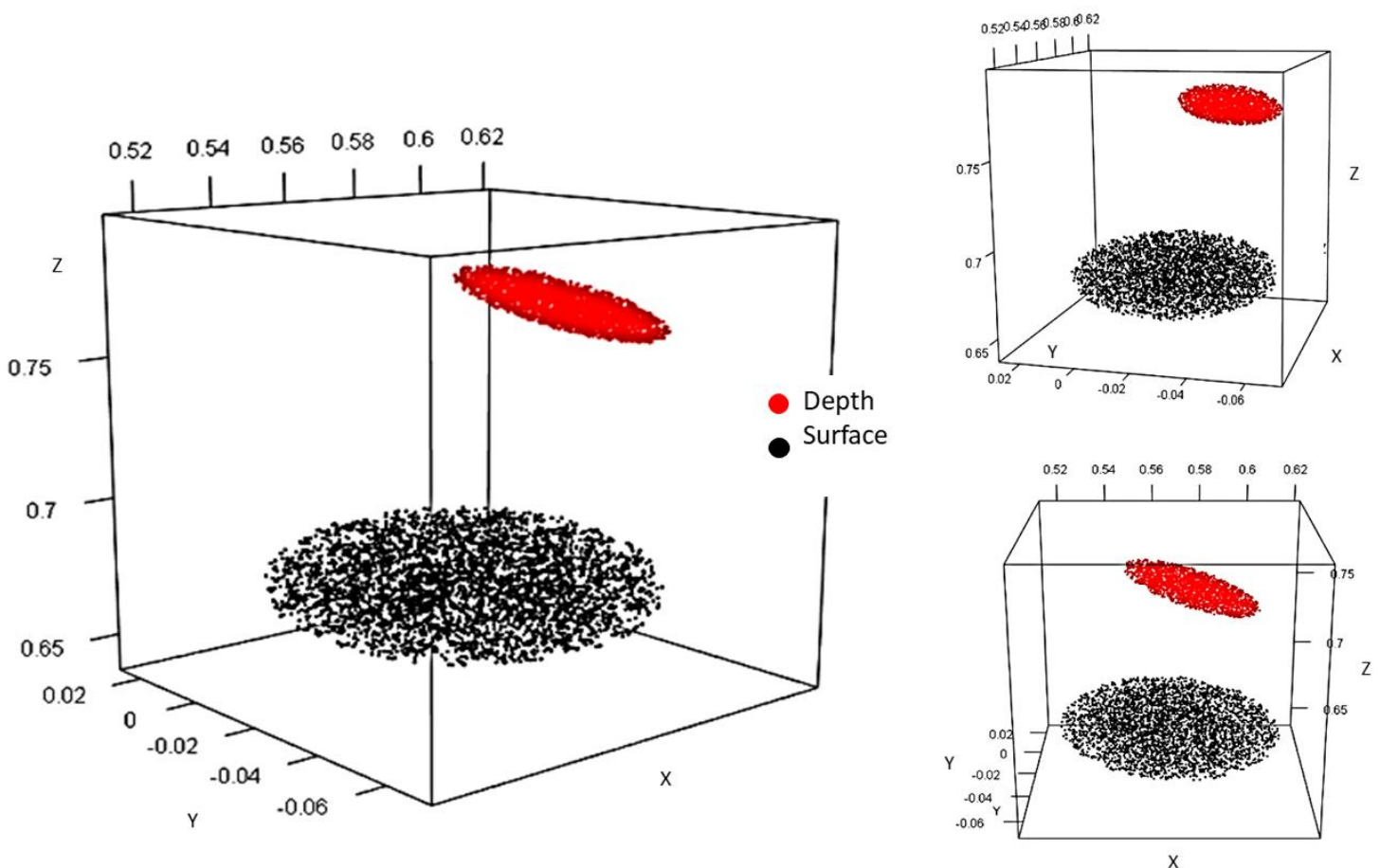

Fig. 12 Graphic representation of confidence ellipse of foliation data showing the different views and the three axes of the direction cosines, the red points concentration is the simulated data based on real depth data and the black points concentration is the simulation data based on real surface data
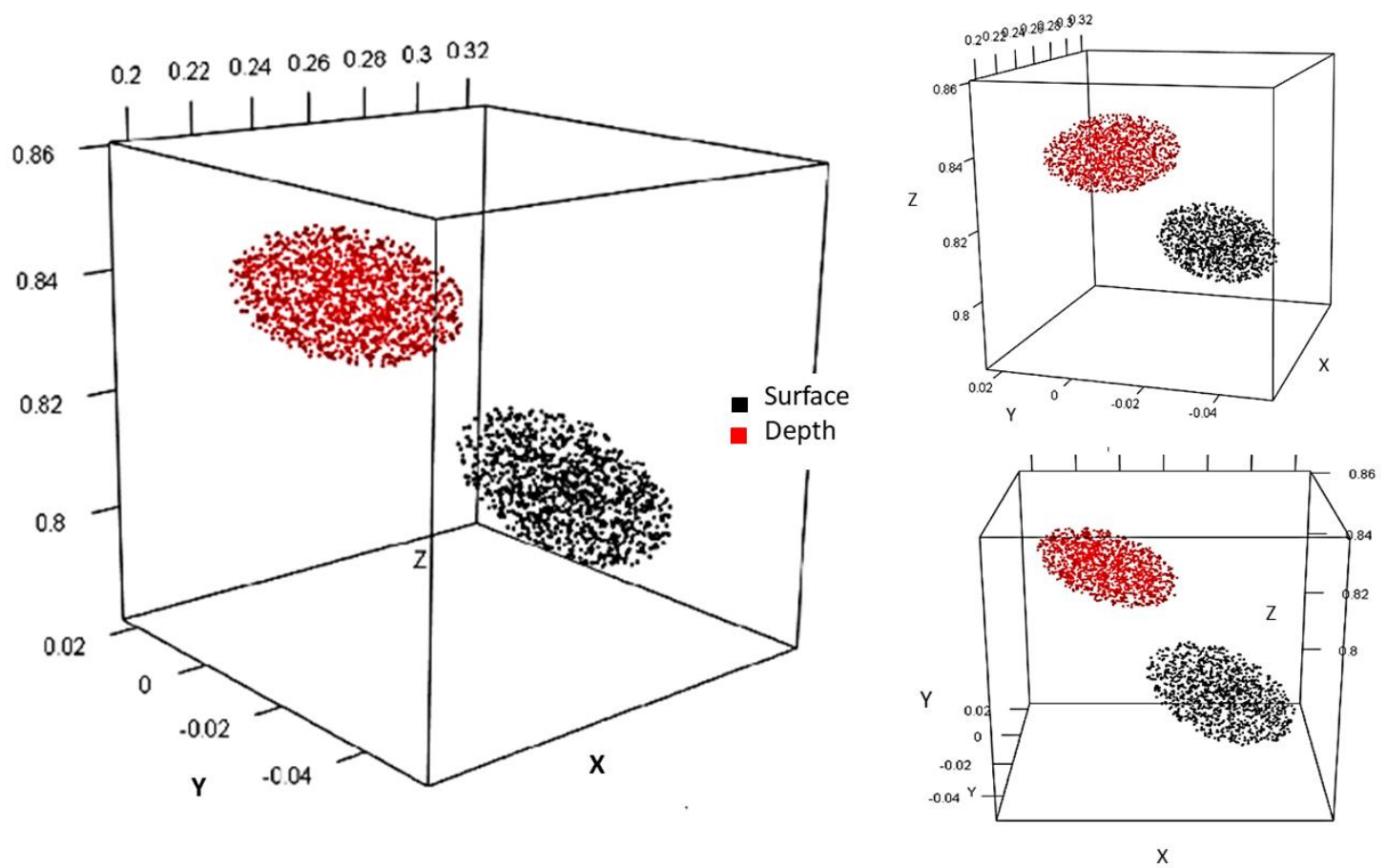

Fig. 13 Graphic representation of confidence ellipse of joints data showing the different views and the three axes of the direction cosines, the red points concentration is the simulated data based on real depth data and the black points concentration is the simulation data based on real surface data obtained. It is possible to simulate a model using the Monte Carlo methods and estimate the depth conditions. It is 
important to accentuate that simulation is possible for the one geological unit, all these analyzes were done for the schist of Araxá Group, rocks that compose the works area.

\section{Discussion}

Initially, the mean values were obtained for all structures set without distinction between foliation and joint. The result of this analysis gives us a general idea of the spatial arrangement of the structures present in the studied massif. Despite the described analyses to show a certain similarity between the surface and depth data, mainly concerning the direction, these structures should be discussed separately to highlight the differences between them. To understand this contrast and apply the Monte Carlo simulation, the foliation and joints were examined separately, distinguishing the position of the measures on the surface and in-depth.

The directions of joints are not evident when all the measurements are plotted together in the same diagram (Fig.5a and 5 b), but we can distinguish only two main systems ( $J 1$ and $J 2$ ). When were separated foliation and joints, it is possible to identify $J 1$ and $J 2$ sets plus two sets with less concentration named $J 3$ and $J 4$ sets (Fig.5c and $5 \mathrm{~d}$ ) when the planar structures, obtained in surface and depth, are analyzed together.

At different depth intervals, the mean values of the foliation orientations were calculated, the former shows no significant variation in direction and plunge along with the depth (Fig.7b and 7d). When we analyze the joint orientation, we remark the appearance of the system (J4) with higher concentration occur in the interval of 41 to $60 \mathrm{~m}$ of depth (Fig.8d), which has not been identified easily on the surface and the 0 to $40 \mathrm{~m}$ of depth due to a less concentration (Fig.8b). Compared to other systems, J4 set has a longitudinal direction to J2//fol with an opposite plunge, suggesting the presence of folds (Fig. 14).

The angle between $J 1$ and $J 3$ systems is $\sim 60$ degrees and could be related to a conjugate system. The spatial arrangement of the conjugate system enables the identification of the $\sigma 1$ direction, that correspondent to the E-W direction, coherent with the N-S direction of the foliation planes (Fig.15).

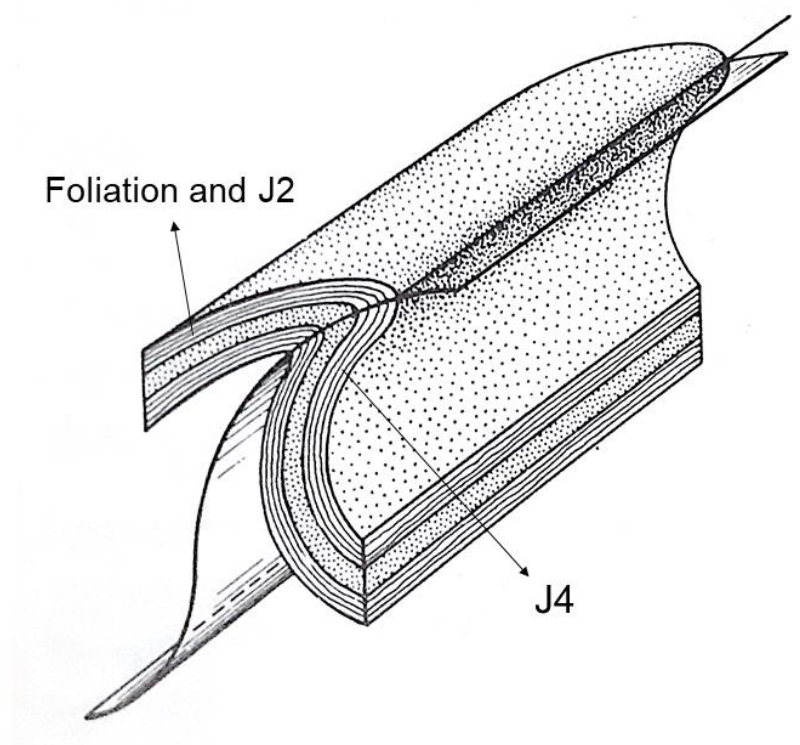




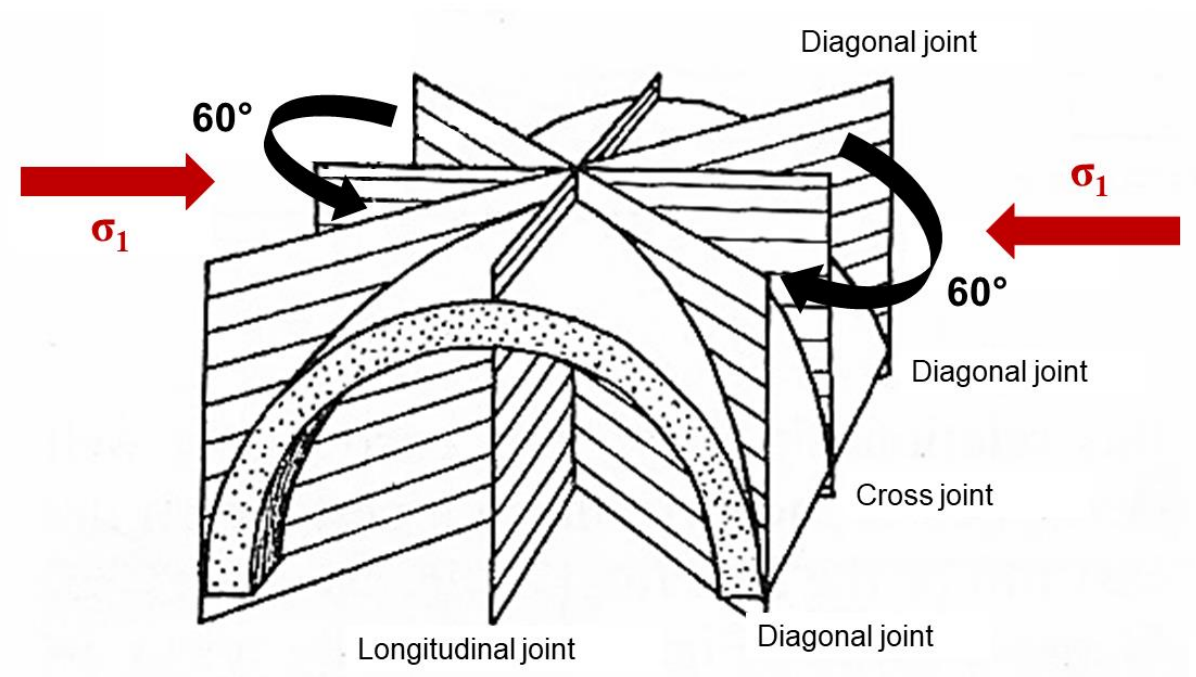

Figure 15. Arrangement of joints and the conjugate system model.

From the average of the values, new data were created from simulated direction cosines, allowing to compare surface and depth data, checking the correspondence between them, and calculating the percentage of this correspondence.

The question is: Is it possible to know or evaluate the data of these structures in depth using Monte Carlo simulation? It is important to emphasize that the simulation was carried out in only a single geological unit (Schist Araxá), as described above. Comparing the behavior of the foliation data with those created by simulation allowed us to know how close or different these data are. For example, in the foliation simulation data (Fig. 12) the similarity is around 96\%, and for joint data, the difference is also low, showing similarity of round $95 \%$ (Fig. 13). These results are suggesting, favorably to use the simulation for optimization of prediction of geological conditions.

The low difference between the spatial orientations of the structures in surface and depth attest that the simulation results presented highlight the feasibility of using the Monte Carlo simulation, to understand the geological structures behavior from the surface to the depth.

\section{Conclusion}

Descriptive analysis shows the importance of understanding the configuration of the geological structures of rock massif, and employing description of data it is possible to identify some differences and/or similarities that allowed to make a correlation between surface and depth data through Monte Carlo simulation.

311 The foliation measures of different depth intervals are quite similar to surface measures, indicating that the direction and plunge of foliation remain remarkably similar from the surface to a depth of 60 meters, only a $4 \%$ difference can be determined through simulation. The joints measures also show a small difference among the distinct levels, however, the short difference between joints surface and depth data, as shown by the simulation, probably can be attributed to the appearance of fractures due to detonations for tunnel opening.

The simulation data using Monte Carlo show that is possible to obtain information and create correlations between surface and depth data and estimate the percentage of correspondence between them. The use of Monte Carlo simulation proves to be a powerful and promising tool in geological investigations for large engineering enterprises (projects). In a future project, we intend to apply this simulation in different units and observe its behavior. 
321 Bruna Catarino Xavier was responsible to write the paper, and compilation of data, and constructing statistical analyzes.

322 Marcos Egydio-Silva contributed to writing the paper, especially in geology structural.

323 Georg Robert Sadowski contributed to write the paper and compilation of data.

324 Bruno de Assis da Silva contributed to constructing statistical analyzes.

325 Victor Junji Takara contributed to constructing statistical analyzes.

Declaration of interests

327 The authors declare that they have no known competing financial interests or personal relationships that could have appeared to influence the work reported in this paper. 


\begin{tabular}{|c|c|c|c|c|c|c|c|c|}
\hline \multirow{2}{*}{ depth (meter) } & \multicolumn{2}{|c|}{ measures } & \multirow{2}{*}{$\theta$} & \multirow{2}{*}{$\phi$} & \multicolumn{3}{|c|}{ cosine director } & \multirow{2}{*}{$\begin{array}{l}\text { geological } \\
\text { structure }\end{array}$} \\
\hline & dip & dipdirection & & & $\mathbf{x}$ & $\mathbf{y}$ & $\mathbf{z}$ & \\
\hline $0-10$ & 53 & 280 & 0,645771823 & 1,745329252 & 0,592672101 & $-0,104504082$ & 0,79863551 & foliation \\
\hline 10 & 56 & 274 & 0,593411946 & 1,640609497 & 0,557830738 & $-0,039007325$ & 0,829037573 & foliation \\
\hline 10 & 25 & 264 & 1,134464014 & 1,466076572 & 0,901342938 & 0,09473496 & 0,422618262 & joint \\
\hline 10 & 83 & 22 & 0,122173048 & $-2,757620218$ & $-0,04565306$ & $-0,112995288$ & 0,992546152 & joint \\
\hline 10 & 68 & 270 & 0,383972435 & 1,570796327 & 0,374606593 & $2,29474 \mathrm{E}-17$ & 0,927183855 & foliation \\
\hline 10 & 55 & 278 & 0,610865238 & 1,710422667 & 0,56799443 & $-0,079826411$ & 0,819152044 & foliation \\
\hline 10 & 60 & 255 & 0,523598776 & 1,308996939 & 0,482962913 & 0,129409523 & 0,866025404 & foliation \\
\hline 10 & 44 & 299 & 0,802851456 & 2,07694181 & 0,629148766 & $-0,348742855$ & 0,69465837 & joint \\
\hline 10 & 33 & 291 & 0,994837674 & 1,93731547 & 0,782966427 & $-0,300552652$ & 0,544639035 & joint \\
\hline 10 & 62 & 258 & 0,488692191 & 1,361356817 & 0,459212483 & 0,097608626 & 0,882947593 & foliation \\
\hline 10 & 89 & 218 & 0,017453293 & 0,663225116 & 0,010744774 & 0,013752684 & 0,999847695 & joint \\
\hline 10 & 80 & 350 & 0,174532925 & 2,967059728 & 0,03015369 & $-0,171010072$ & 0,984807753 & joint \\
\hline 10 & 76 & 322 & 0,244346095 & 2,478367538 & 0,148941991 & $-0,190637055$ & 0,970295726 & joint \\
\hline 10 & 89 & 108 & 0,017453293 & $-1,256637061$ & $-0,016598225$ & 0,00539309 & 0,999847695 & joint \\
\hline 10 & 62 & 200 & 0,488692191 & 0,34906585 & 0,160568731 & 0,441158963 & 0,882947593 & joint \\
\hline 10 & 47 & 279 & 0,750491578 & 1,727875959 & 0,673601829 & $-0,106688049$ & 0,731353702 & joint \\
\hline 10 & 83 & 202 & 0,122173048 & 0,383972435 & 0,04565306 & 0,112995288 & 0,992546152 & joint \\
\hline 10 & 62 & 330 & 0,488692191 & 2,617993878 & 0,234735781 & $-0,4065743$ & 0,882947593 & joint \\
\hline 10 & 33 & 291 & 0,994837674 & 1,93731547 & 0,782966427 & $-0,300552652$ & 0,544639035 & joint \\
\hline 10 & 45 & 268 & 0,785398163 & 1,535889742 & 0,706676031 & 0,024677671 & 0,707106781 & joint \\
\hline 10 & 71 & 218 & 0,331612558 & 0,663225116 & 0,20043977 & 0,256551207 & 0,945518576 & joint \\
\hline 10 & 52 & 268 & 0,663225116 & 1,535889742 & 0,615286431 & 0,021486276 & 0,788010754 & joint \\
\hline 10 & 56 & 73 & 0,593411946 & $-1,8675023$ & $-0,534758833$ & $-0,163492182$ & 0,829037573 & joint \\
\hline 10 & 84 & 32 & 0,104719755 & $-2,583087293$ & $-0,055391646$ & $-0,088645164$ & 0,994521895 & joint \\
\hline 10 & 89 & 198 & 0,017453293 & 0,314159265 & 0,00539309 & 0,016598225 & 0,999847695 & joint \\
\hline 10 & 74 & 236 & 0,27925268 & 0,977384381 & 0,228513724 & 0,154134453 & 0,961261696 & joint \\
\hline 10 & 22 & 124 & 1,186823891 & $-0,977384381$ & $-0,768670252$ & 0,518474632 & 0,374606593 & joint \\
\hline 10 & 51 & 358 & 0,680678408 & 3,106686069 & 0,021962965 & $-0,628937026$ & 0,777145961 & joint \\
\hline 10 & 38 & 165 & 0,907571211 & $-0,261799388$ & $-0,203952191$ & 0,761159938 & 0,615661475 & joint \\
\hline 10 & 53 & 282 & 0,645771823 & 1,780235837 & 0,5886663921 & $-0,125124379$ & 0,79863551 & joint \\
\hline 10 & 73 & 208 & 0,296705973 & 0,488692191 & 0,137260201 & 0,258148893 & 0,956304756 & joint \\
\hline 10 & 41 & 267 & 0,855211333 & 1,518436449 & 0,753675277 & 0,039498448 & 0,656059029 & joint \\
\hline 10 & 48 & 291 & 0,733038286 & 1,93731547 & 0,624687237 & $-0,239794963$ & 0,743144825 & joint \\
\hline 10 & 23 & 152 & 1,169370599 & $-0,488692191$ & $-0,432150852$ & 0,812757545 & 0,390731128 & joint \\
\hline $11-20$ & 70 & 275 & 0,34906585 & 1,658062789 & 0,340718653 & $-0,02980902$ & 0,939692621 & foliation \\
\hline 20 & 62 & 206 & 0,488692191 & 0,453785606 & 0,205802787 & 0,421958246 & 0,882947593 & foliation \\
\hline 20 & 46 & 318 & 0,767944871 & 2,408554368 & 0,464817177 & $-0,516231773$ & 0,7193398 & joint \\
\hline 20 & 68 & 275 & 0,383972435 & 1,658062789 & 0,373181102 & $-0,032649116$ & 0,927183855 & foliation \\
\hline 20 & 65 & 274 & 0,436332313 & 1,640609497 & 0,421588785 & $-0,02948036$ & 0,906307787 & foliation \\
\hline 20 & 72 & 269 & 0,314159265 & 1,553343034 & 0,30896993 & 0,00539309 & 0,951056516 & foliation \\
\hline 20 & 50 & 274 & 0,698131701 & 1,640609497 & 0,641221811 & $-0,044838597$ & 0,766044443 & foliation \\
\hline 20 & 58 & 275 & 0,558505361 & 1,658062789 & 0,527902761 & $-0,046185507$ & 0,848048096 & foliation \\
\hline 20 & 86 & 341 & 0,06981317 & 2,809980096 & 0,022710486 & $-0,065956042$ & 0,99756405 & joint \\
\hline 20 & 73 & 274 & 0,296705973 & 1,640609497 & 0,291659502 & $-0,020394819$ & 0,956304756 & joint \\
\hline 20 & 58 & 254 & 0,558505361 & 1,291543646 & 0,509391091 & 0,146065545 & 0,848048096 & foliation \\
\hline 20 & 85 & 258 & 0,087266463 & 1,361356817 & 0,085251181 & 0,018120698 & 0,996194698 & foliation \\
\hline 20 & 89 & 275 & 0,017453293 & 1,658062789 & 0,017385995 & $-0,001521077$ & 0,999847695 & foliation \\
\hline 20 & 88 & 276 & 0,034906585 & 1,675516082 & 0,034708314 & $-0,003647991$ & 0,999390827 & foliation \\
\hline 20 & 60 & 278 & 0,523598776 & 1,710422667 & 0,495134034 & $-0,06958655$ & 0,866025404 & foliation \\
\hline 20 & 58 & 282 & 0,558505361 & 1,780235837 & 0,518339257 & $-0,11017641$ & 0,848048096 & foliation \\
\hline 20 & 52 & 297 & 0,663225116 & 2,042035225 & 0,548558391 & $-0,279504461$ & 0,788010754 & foliation \\
\hline 20 & 81 & 346 & 0,157079633 & 2,897246558 & 0,037844922 & $-0,151787693$ & 0,987688341 & joint \\
\hline 20 & 80 & 154 & 0,174532925 & $-0,453785606$ & $-0,076122351$ & 0,156073948 & 0,984807753 & joint \\
\hline 20 & 60 & 13 & 0,523598776 & $-2,914699851$ & $-0,112475527$ & $-0,487185032$ & 0,866025404 & joint \\
\hline 20 & 78 & 337 & 0,20943951 & 2,740166926 & 0,08123757 & $-0,19138372$ & 0,978147601 & joint \\
\hline 20 & 89 & 110 & 0,017453293 & $-1,221730476$ & $-0,016399898$ & 0,005969075 & 0,999847695 & joint \\
\hline
\end{tabular}




\begin{tabular}{|c|c|c|c|c|c|c|c|c|}
\hline \multirow{2}{*}{ depth (meter) } & \multicolumn{2}{|c|}{ measures } & \multirow{2}{*}{$\theta$} & \multirow{2}{*}{$\phi$} & \multicolumn{3}{|c|}{ cosine director } & \multirow{2}{*}{$\begin{array}{l}\text { geological } \\
\text { structure }\end{array}$} \\
\hline & $\operatorname{dip}$ & dipdirection & & & $\mathbf{x}$ & $\mathbf{y}$ & $\mathbf{z}$ & \\
\hline 20 & 57 & 80 & 0,575958653 & $-1,745329252$ & $-0,536364744$ & $-0,094575576$ & 0,838670568 & joint \\
\hline 20 & 65 & 263 & 0,436332313 & 1,448623279 & 0,419468129 & 0,05150421 & 0,906307787 & foliation \\
\hline 20 & 43 & 282 & 0,820304748 & 1,780235837 & 0,715371869 & $-0,152056985$ & 0,68199836 & foliation \\
\hline 20 & 70 & 305 & 0,34906585 & 2,181661565 & 0,2801665 & $-0,196174695$ & 0,939692621 & foliation \\
\hline 20 & 70 & 305 & 0,34906585 & 2,181661565 & 0,2801665 & $-0,196174695$ & 0,939692621 & foliation \\
\hline $21-30$ & 50 & 268 & 0,698131701 & 1,535889742 & 0,642396041 & 0,022432964 & 0,766044443 & foliation \\
\hline 30 & 53 & 269 & 0,645771823 & 1,553343034 & 0,601723364 & 0,01050312 & 0,79863551 & foliation \\
\hline 30 & 59 & 275 & 0,541052068 & 1,658062789 & 0,5130782 & $-0,044888526$ & 0,857167301 & foliation \\
\hline 30 & 62 & 240 & 0,488692191 & 1,047197551 & 0,4065743 & 0,234735781 & 0,882947593 & foliation \\
\hline 30 & 65 & 253 & 0,436332313 & 1,274090354 & 0,404151854 & 0,123561622 & 0,906307787 & foliation \\
\hline 30 & 64 & 280 & 0,453785606 & 1,745329252 & 0,431711304 & $-0,076122351$ & 0,898794046 & foliation \\
\hline 30 & 89 & 33 & 0,017453293 & $-2,565634$ & $-0,009505262$ & $-0,01463682$ & 0,999847695 & joint \\
\hline 30 & 30 & 255 & 1,047197551 & 1,308996939 & 0,836516304 & 0,224143868 & 0,5 & foliation \\
\hline 30 & 68 & 250 & 0,383972435 & 1,221730476 & 0,352015052 & 0,128123001 & 0,927183855 & foliation \\
\hline 30 & 80 & 202 & 0,174532925 & 0,383972435 & 0,065049752 & 0,161003787 & 0,984807753 & joint \\
\hline 30 & 85 & 11 & 0,087266463 & $-2,949606436$ & $-0,0166301$ & $-0,085554446$ & 0,996194698 & joint \\
\hline 30 & 42 & 173 & 0,837758041 & $-0,122173048$ & $-0,090566572$ & 0,737605537 & 0,669130606 & joint \\
\hline 30 & 60 & 80 & 0,523598776 & $-1,745329252$ & $-0,492403877$ & $-0,086824089$ & 0,866025404 & joint \\
\hline 30 & 15 & 41 & 1,308996939 & $-2,42600766$ & $-0,63370436$ & $-0,728993475$ & 0,258819045 & joint \\
\hline 30 & 70 & 208 & 0,34906585 & 0,488692191 & 0,160568731 & 0,301985862 & 0,939692621 & joint \\
\hline 30 & 89 & 235 & 0,017453293 & 0,959931089 & 0,014296174 & 0,010010289 & 0,999847695 & joint \\
\hline 30 & 50 & 289 & 0,698131701 & 1,902408885 & 0,607767625 & $-0,209271176$ & 0,766044443 & joint \\
\hline 30 & 86 & 223 & 0,06981317 & 0,750491578 & 0,047573801 & 0,051016655 & 0,99756405 & joint \\
\hline 30 & 89 & 235 & 0,017453293 & 0,959931089 & 0,014296174 & 0,010010289 & 0,999847695 & joint \\
\hline 30 & 78 & 48 & 0,20943951 & $-2,303834613$ & $-0,154508497$ & $-0,139120076$ & 0,978147601 & joint \\
\hline 30 & 30 & 255 & 1,047197551 & 1,308996939 & 0,836516304 & 0,224143868 & 0,5 & foliation \\
\hline 30 & 86 & 223 & 0,06981317 & 0,750491578 & 0,047573801 & 0,051016655 & 0,99756405 & foliation \\
\hline 30 & 80 & 202 & 0,174532925 & 0,383972435 & 0,065049752 & 0,161003787 & 0,984807753 & joint \\
\hline 30 & 85 & 11 & 0,087266463 & $-2,949606436$ & $-0,0166301$ & $-0,085554446$ & 0,996194698 & joint \\
\hline 30 & 15 & 141 & 1,308996939 & $-0,680678408$ & $-0,607876819$ & 0,750665355 & 0,258819045 & joint \\
\hline 30 & 70 & 208 & 0,34906585 & 0,488692191 & 0,160568731 & 0,301985862 & 0,939692621 & joint \\
\hline 30 & 89 & 235 & 0,017453293 & 0,959931089 & 0,014296174 & 0,010010289 & 0,999847695 & joint \\
\hline 30 & 50 & 289 & 0,698131701 & 1,902408885 & 0,607767625 & $-0,209271176$ & 0,766044443 & joint \\
\hline 30 & 53 & 200 & 0,645771823 & 0,34906585 & 0,20583286 & 0,565521136 & 0,79863551 & joint \\
\hline 30 & 60 & 80 & 0,523598776 & $-1,745329252$ & $-0,492403877$ & $-0,086824089$ & 0,866025404 & joint \\
\hline 30 & 50 & 280 & 0,698131701 & 1,745329252 & 0,633022222 & $-0,111618897$ & 0,766044443 & foliation \\
\hline 30 & 54 & 280 & 0,628318531 & 1,745329252 & 0,578855474 & $-0,102067838$ & 0,809016994 & foliation \\
\hline 30 & 42 & 285 & 0,837758041 & 1,832595715 & 0,71782278 & $-0,192340034$ & 0,669130606 & foliation \\
\hline 30 & 57 & 286 & 0,575958653 & 1,850049007 & 0,523540642 & $-0,150122863$ & 0,838670568 & foliation \\
\hline 30 & 50 & 290 & 0,698131701 & 1,919862177 & 0,604022774 & $-0,21984631$ & 0,766044443 & foliation \\
\hline 30 & 63 & 295 & 0,471238898 & 2,00712864 & 0,411455125 & $-0,191864676$ & 0,891006524 & foliation \\
\hline 30 & 88 & 15 & 0,034906585 & $-2,879793266$ & $-0,009032654$ & $-0,033710325$ & 0,999390827 & joint \\
\hline 30 & 53 & 70 & 0,645771823 & $-1,919862177$ & $-0,565521136$ & $-0,20583286$ & 0,79863551 & joint \\
\hline 30 & 55 & 88 & 0,610865238 & $-1,605702912$ & $-0,573227029$ & $-0,020017529$ & 0,819152044 & joint \\
\hline 30 & 82 & 150 & 0,13962634 & $-0,523598776$ & $-0,06958655$ & 0,120527441 & 0,990268069 & joint \\
\hline 30 & 81 & 195 & 0,157079633 & 0,261799388 & 0,040488219 & 0,15110409 & 0,987688341 & joint \\
\hline 30 & 50 & 272 & 0,698131701 & 1,605702912 & 0,642396041 & $-0,022432964$ & 0,766044443 & joint \\
\hline 30 & 83 & 360 & 0,122173048 & 3,141592654 & $1,49308 \mathrm{E}-17$ & $-0,121869343$ & 0,992546152 & joint \\
\hline 30 & 78 & 208 & 0,20943951 & 0,488692191 & 0,097608626 & 0,183575127 & 0,978147601 & joint \\
\hline 30 & 63 & 95 & 0,471238898 & $-1,483529864$ & $-0,452262929$ & 0,039567879 & 0,891006524 & joint \\
\hline 30 & 57 & 287 & 0,575958653 & 1,8675023 & 0,520840899 & $-0,159237043$ & 0,838670568 & foliation \\
\hline 30 & 59 & 295 & 0,541052068 & 2,00712864 & 0,466783018 & $-0,217664496$ & 0,857167301 & foliation \\
\hline 30 & 52 & 297 & 0,663225116 & 2,042035225 & 0,548558391 & $-0,279504461$ & 0,788010754 & foliation \\
\hline 30 & 87 & 23 & 0,052359878 & $-2,740166926$ & $-0,020449287$ & $-0,048175502$ & 0,998629535 & joint \\
\hline 30 & 50 & 293 & 0,698131701 & 1,972222055 & 0,591689114 & $-0,251157128$ & 0,766044443 & foliation \\
\hline 30 & 30 & 255 & 1,047197551 & 1,308996939 & 0,836516304 & 0,224143868 & 0,5 & foliation \\
\hline 30 & 53 & 283 & 0,645771823 & 1,79768913 & 0,586390543 & $-0,135378924$ & 0,79863551 & foliation \\
\hline
\end{tabular}




\begin{tabular}{|c|c|c|c|c|c|c|c|c|}
\hline \multirow{2}{*}{ depth (meter) } & \multicolumn{2}{|c|}{ measures } & \multirow{2}{*}{$\theta$} & \multirow{2}{*}{$\phi$} & \multicolumn{3}{|c|}{ cosine director } & \multirow{2}{*}{$\begin{array}{l}\text { geological } \\
\text { structure }\end{array}$} \\
\hline & $\operatorname{dip}$ & dipdirection & & & $\mathbf{x}$ & $\mathbf{y}$ & $\mathbf{z}$ & \\
\hline 30 & 40 & 273 & 0,872664626 & 1,623156204 & 0,764994606 & $-0,040091668$ & 0,64278761 & foliation \\
\hline 30 & 87 & 352 & 0,052359878 & 3,001966313 & 0,007283757 & $-0,051826626$ & 0,998629535 & joint \\
\hline 30 & 80 & 40 & 0,174532925 & $-2,443460953$ & $-0,111618897$ & $-0,133022222$ & 0,984807753 & joint \\
\hline 30 & 70 & 45 & 0,34906585 & $-2,35619449$ & $-0,241844763$ & $-0,241844763$ & 0,939692621 & joint \\
\hline 30 & 78 & 48 & 0,20943951 & $-2,303834613$ & \begin{tabular}{|l|}
$-0,154508497$ \\
\end{tabular} & $-0,139120076$ & 0,978147601 & joint \\
\hline 30 & 88 & 223 & 0,034906585 & 0,750491578 & 0,0238014 & 0,025523876 & 0,999390827 & joint \\
\hline 30 & 63 & 162 & 0,471238898 & $-0,314159265$ & $-0,14029078$ & 0,431770623 & 0,891006524 & joint \\
\hline 30 & 50 & 293 & 0,698131701 & 1,972222055 & 0,591689114 & $-0,251157128$ & 0,766044443 & foliation \\
\hline 30 & 68 & 250 & 0,383972435 & 1,221730476 & 0,352015052 & 0,128123001 & 0,927183855 & foliation \\
\hline 30 & 40 & 273 & 0,872664626 & 1,623156204 & 0,764994606 & $-0,040091668$ & 0,64278761 & foliation \\
\hline 30 & 53 & 283 & 0,645771823 & 1,79768913 & 0,586390543 & $-0,135378924$ & 0,79863551 & foliation \\
\hline 30 & 87 & 352 & 0,052359878 & 3,001966313 & 0,007283757 & $-0,051826626$ & 0,998629535 & joint \\
\hline 30 & 80 & 40 & 0,174532925 & $-2,443460953$ & $-0,111618897$ & $-0,133022222$ & 0,984807753 & joint \\
\hline 30 & 70 & 45 & 0,34906585 & $-2,35619449$ & $-0,241844763$ & $-0,241844763$ & 0,939692621 & joint \\
\hline 30 & 88 & 223 & 0,034906585 & 0,750491578 & 0,0238014 & 0,025523876 & 0,999390827 & joint \\
\hline 30 & 78 & 48 & 0,20943951 & $-2,303834613$ & $-0,154508497$ & $-0,139120076$ & 0,978147601 & joint \\
\hline 30 & 45 & 81 & 0,785398163 & $-1,727875959$ & $-0,698401123$ & $-0,110615871$ & 0,707106781 & joint \\
\hline 30 & 63 & 162 & 0,471238898 & $-0,314159265$ & $-0,14029078$ & 0,431770623 & 0,891006524 & joint \\
\hline 30 & 50 & 268 & 0,698131701 & 1,535889742 & 0,642396041 & 0,022432964 & 0,766044443 & foliation \\
\hline 30 & 45 & 280 & 0,785398163 & 1,745329252 & 0,69636424 & $-0,122787804$ & 0,707106781 & foliation \\
\hline 30 & 42 & 288 & 0,837758041 & 1,884955592 & 0,706772729 & $-0,22964438$ & 0,669130606 & foliation \\
\hline 30 & 31 & 299 & 1,029744259 & 2,07694181 & 0,749695414 & $-0,415562954$ & 0,515038075 & foliation \\
\hline 30 & 78 & 18 & 0,20943951 & $-2,827433388$ & $-0,064248246$ & $-0,197735768$ & 0,978147601 & joint \\
\hline 30 & 60 & 22 & 0,523598776 & $-2,757620218$ & $-0,187303297$ & $-0,463591927$ & 0,866025404 & joint \\
\hline 30 & 58 & 73 & 0,558505361 & $-1,8675023$ & $-0,506764313$ & $-0,154933399$ & 0,848048096 & joint \\
\hline 30 & 60 & 90 & 0,523598776 & $-1,570796327$ & \begin{tabular}{|c|}
$-0,5$ \\
\end{tabular} & $3,06287 \mathrm{E}-17$ & 0,866025404 & joint \\
\hline 30 & 81 & 188 & 0,157079633 & 0,13962634 & 0,02177147 & 0,154912056 & 0,987688341 & joint \\
\hline 30 & 54 & 200 & 0,628318531 & 0,34906585 & 0,201034396 & 0,552337464 & 0,809016994 & joint \\
\hline 30 & 81 & 223 & 0,157079633 & 0,750491578 & 0,106688049 & 0,114408925 & 0,987688341 & joint \\
\hline 30 & 89 & 343 & 0,017453293 & 2,844886681 & 0,00510259 & $-0,016689819$ & 0,999847695 & joint \\
\hline 30 & 86 & 141 & 0,06981317 & $-0,680678408$ & $-0,043899171$ & 0,054210962 & 0,99756405 & joint \\
\hline 30 & 85 & 348 & 0,087266463 & 2,932153143 & 0,018120698 & $-0,085251181$ & 0,996194698 & joint \\
\hline 30 & 89 & 350 & 0,017453293 & 2,967059728 & 0,003030579 & $-0,017187265$ & 0,999847695 & joint \\
\hline 30 & 40 & 278 & 0,872664626 & 1,710422667 & 0,758589351 & $-0,106612781$ & 0,64278761 & foliation \\
\hline 30 & 88 & 33 & 0,034906585 & $-2,565634$ & $-0,019007628$ & $-0,029269181$ & 0,999390827 & joint \\
\hline 30 & 77 & 348 & 0,226892803 & 2,932153143 & 0,046769954 & $-0,220035334$ & 0,974370065 & joint \\
\hline 30 & 73 & 227 & 0,296705973 & 0,820304748 & 0,213827128 & 0,199397023 & 0,956304756 & joint \\
\hline 30 & 89 & 225 & 0,017453293 & 0,785398163 & 0,012340715 & 0,012340715 & 0,999847695 & joint \\
\hline 30 & 45 & 284 & 0,785398163 & 1,815142422 & 0,686102688 & $-0,171064613$ & 0,707106781 & joint \\
\hline 30 & 62 & 189 & 0,488692191 & 0,157079633 & 0,073441533 & 0,463691589 & 0,882947593 & joint \\
\hline 30 & 88 & 33 & 0,034906585 & $-2,565634$ & $-0,019007628$ & $-0,029269181$ & 0,999390827 & joint \\
\hline 30 & 89 & 225 & 0,017453293 & 0,785398163 & 0,012340715 & 0,012340715 & 0,999847695 & joint \\
\hline 30 & 40 & 278 & 0,872664626 & 1,710422667 & 0,758589351 & $-0,106612781$ & 0,64278761 & joint \\
\hline 30 & 77 & 348 & 0,226892803 & 2,932153143 & 0,046769954 & $-0,220035334$ & 0,974370065 & joint \\
\hline 30 & 45 & 284 & 0,785398163 & 1,815142422 & 0,686102688 & $-0,171064613$ & 0,707106781 & joint \\
\hline 30 & 73 & 227 & 0,296705973 & 0,820304748 & 0,213827128 & 0,199397023 & 0,956304756 & joint \\
\hline 30 & 62 & 189 & 0,488692191 & 0,157079633 & 0,073441533 & 0,463691589 & 0,882947593 & joint \\
\hline 30 & 42 & 288 & 0,837758041 & 1,884955592 & 0,706772729 & $-0,22964438$ & 0,669130606 & foliation \\
\hline 30 & 53 & 287 & 0,645771823 & 1,8675023 & 0,575518569 & $-0,175953684$ & 0,79863551 & foliation \\
\hline 30 & 52 & 315 & 0,663225116 & 2,35619449 & 0,435338404 & $-0,435338404$ & 0,788010754 & foliation \\
\hline 30 & 80 & 18 & 0,174532925 & $-2,827433388$ & $-0,053660238$ & $-0,165149231$ & 0,984807753 & joint \\
\hline 30 & 54 & 27 & 0,628318531 & $-2,670353756$ & $-0,26684892$ & $-0,523720495$ & 0,809016994 & joint \\
\hline 30 & 74 & 33 & 0,27925268 & $-2,565634$ & $-0,150122863$ & $-0,231168938$ & 0,961261696 & joint \\
\hline 30 & 84 & 70 & 0,104719755 & $-1,919862177$ & $-0,098224626$ & $-0,03575084$ & 0,994521895 & joint \\
\hline 30 & 88 & 87 & 0,034906585 & $-1,623156204$ & $-0,034851668$ & $-0,001826499$ & 0,999390827 & joint \\
\hline 30 & 89 & 90 & 0,017453293 & $-1,570796327$ & $-0,017452406$ & $1,06909 \mathrm{E}-18$ & 0,999847695 & joint \\
\hline 30 & 70 & 113 & 0,34906585 & $-1,169370599$ & $-0,314831202$ & 0,133637917 & 0,939692621 & joint \\
\hline
\end{tabular}




\begin{tabular}{|c|c|c|c|c|c|c|c|c|}
\hline \multirow{2}{*}{ depth (meter) } & \multicolumn{2}{|c|}{ measures } & \multirow{2}{*}{$\theta$} & \multirow{2}{*}{$\phi$} & \multicolumn{3}{|c|}{ cosine director } & \multirow{2}{*}{$\begin{array}{l}\text { geological } \\
\text { structure }\end{array}$} \\
\hline & $\operatorname{dip}$ & dipdirection & & & $\mathbf{x}$ & $\mathbf{y}$ & $\mathbf{z}$ & \\
\hline 30 & 82 & 198 & 0,13962634 & 0,314159265 & 0,043006853 & 0,132361485 & 0,990268069 & joint \\
\hline 30 & 79 & 208 & 0,191986218 & 0,488692191 & 0,089579397 & 0,168474343 & 0,981627183 & joint \\
\hline 30 & 84 & 209 & 0,104719755 & 0,506145483 & 0,050676405 & 0,091422654 & 0,994521895 & joint \\
\hline 30 & 80 & 270 & 0,174532925 & 1,570796327 & 0,173648178 & $1,06372 \mathrm{E}-17$ & 0,984807753 & joint \\
\hline 30 & 67 & 347 & 0,401425728 & 2,914699851 & 0,087895379 & $-0,380716715$ & 0,920504853 & joint \\
\hline 30 & 61 & 292 & 0,506145483 & 1,954768762 & 0,449507652 & $-0,18161288$ & 0,874619707 & joint \\
\hline $31-40$ & 38 & 292 & 0,907571211 & 1,954768762 & 0,730630848 & $-0,295194024$ & 0,615661475 & foliation \\
\hline 40 & 50 & 272 & 0,698131701 & 1,605702912 & 0,642396041 & $-0,022432964$ & 0,766044443 & foliation \\
\hline 40 & 89 & 225 & 0,017453293 & 0,785398163 & 0,012340715 & 0,012340715 & 0,999847695 & joint \\
\hline 40 & 88 & 32 & 0,034906585 & $-2,583087293$ & $-0,018493916$ & $-0,029596452$ & 0,999390827 & joint \\
\hline 40 & 88 & 354 & 0,034906585 & 3,036872898 & 0,003647991 & $-0,034708314$ & 0,999390827 & joint \\
\hline 40 & 38 & 292 & 0,907571211 & 1,954768762 & 0,730630848 & $-0,295194024$ & 0,615661475 & foliation \\
\hline 40 & 50 & 272 & 0,698131701 & 1,605702912 & 0,642396041 & $-0,022432964$ & 0,766044443 & foliation \\
\hline 40 & 40 & 282 & 0,872664626 & 1,780235837 & 0,749304534 & $-0,159269595$ & 0,64278761 & foliation \\
\hline 40 & 88 & 354 & 0,034906585 & 3,036872898 & 0,003647991 & $-0,034708314$ & 0,999390827 & joint \\
\hline 40 & 88 & 32 & 0,034906585 & $-2,583087293$ & $-0,018493916$ & $-0,029596452$ & 0,999390827 & joint \\
\hline 40 & 42 & 254 & 0,837758041 & 1,291543646 & 0,714356655 & 0,204838475 & 0,669130606 & foliation \\
\hline 40 & 62 & 274 & 0,488692191 & 1,640609497 & 0,468327954 & $-0,032748681$ & 0,882947593 & foliation \\
\hline 40 & 40 & 276 & 0,872664626 & 1,675516082 & 0,761847972 & $-0,080073448$ & 0,64278761 & foliation \\
\hline 40 & 54 & 27 & 0,628318531 & $-2,670353756$ & $-0,26684892$ & $-0,523720495$ & 0,809016994 & joint \\
\hline 40 & 89 & 90 & 0,017453293 & $-1,570796327$ & $-0,017452406$ & $1,06909 \mathrm{E}-18$ & 0,999847695 & joint \\
\hline 40 & 84 & 70 & 0,104719755 & $-1,919862177$ & $-0,098224626$ & $-0,03575084$ & 0,994521895 & joint \\
\hline 40 & 68 & 109 & 0,383972435 & $-1,239183769$ & $-0,354197493$ & 0,121959977 & 0,927183855 & joint \\
\hline 40 & 70 & 260 & 0,34906585 & 1,396263402 & 0,336824089 & 0,059391175 & 0,939692621 & joint \\
\hline 40 & 75 & 320 & 0,261799388 & 2,443460953 & 0,166365675 & $-0,198266891$ & 0,965925826 & joint \\
\hline 40 & 89 & 328 & 0,017453293 & 2,583087293 & 0,009248366 & $-0,01480048$ & 0,999847695 & joint \\
\hline 40 & 68 & 342 & 0,383972435 & 2,827433388 & 0,115759804 & $-0,356272042$ & 0,927183855 & joint \\
\hline 40 & 64 & 67 & 0,453785606 & $-1,972222055$ & $-0,403522768$ & $-0,171285253$ & 0,898794046 & joint \\
\hline 40 & 53 & 281 & 0,645771823 & 1,762782545 & 0,590757986 & $-0,11483172$ & 0,79863551 & joint \\
\hline 40 & 57 & 266 & 0,575958653 & 1,500983157 & 0,543312322 & 0,037992099 & 0,838670568 & joint \\
\hline 40 & 87 & 229 & 0,052359878 & 0,855211333 & 0,039498448 & 0,034335477 & 0,998629535 & joint \\
\hline 40 & 89 & 167 & 0,017453293 & $-0,226892803$ & $-0,003925937$ & 0,017005102 & 0,999847695 & joint \\
\hline 40 & 19 & 162 & 1,239183769 & $-0,314159265$ & $-0,292181308$ & 0,899241603 & 0,325568154 & joint \\
\hline 40 & 42 & 260 & 0,837758041 & 1,396263402 & 0,731854786 & 0,129045745 & 0,669130606 & foliation \\
\hline 40 & 54 & 255 & 0,628318531 & 1,308996939 & 0,567756956 & 0,152130018 & 0,809016994 & joint \\
\hline 40 & 60 & 248 & 0,523598776 & 1,186823891 & 0,463591927 & 0,187303297 & 0,866025404 & joint \\
\hline 40 & 80 & 33 & 0,174532925 & $-2,565634$ & $-0,094575576$ & $-0,145633616$ & 0,984807753 & joint \\
\hline 40 & 70 & 43 & 0,34906585 & $-2,391101075$ & $-0,233257177$ & $-0,250137698$ & 0,939692621 & joint \\
\hline 40 & 42 & 260 & 0,837758041 & 1,396263402 & 0,731854786 & 0,129045745 & 0,669130606 & foliation \\
\hline 40 & 60 & 248 & 0,523598776 & 1,186823891 & 0,463591927 & 0,187303297 & 0,866025404 & joint \\
\hline 40 & 54 & 255 & 0,628318531 & 1,308996939 & 0,567756956 & 0,152130018 & 0,809016994 & joint \\
\hline 40 & 70 & 43 & 0,34906585 & $-2,391101075$ & $-0,233257177$ & $-0,250137698$ & 0,939692621 & joint \\
\hline 40 & 45 & 276 & 0,785398163 & 1,675516082 & 0,703233176 & $-0,073912785$ & 0,707106781 & foliation \\
\hline 40 & 62 & 274 & 0,488692191 & 1,640609497 & 0,468327954 & $-0,032748681$ & 0,882947593 & foliation \\
\hline 40 & 75 & 40 & 0,261799388 & $-2,443460953$ & $-0,166365675$ & $-0,198266891$ & 0,965925826 & joint \\
\hline 40 & 44 & 271 & 0,802851456 & 1,588249619 & 0,719230241 & $-0,012554211$ & 0,69465837 & joint \\
\hline 40 & 50 & 305 & 0,698131701 & 2,181661565 & 0,526540785 & $-0,368687826$ & 0,766044443 & joint \\
\hline 40 & 36 & 72 & 0,942477796 & $-1,884955592$ & $-0,769420884$ & $-0,25$ & 0,587785252 & joint \\
\hline 40 & 68 & 142 & 0,383972435 & $-0,663225116$ & $-0,230630848$ & 0,295194024 & 0,927183855 & joint \\
\hline 40 & 58 & 288 & 0,558505361 & 1,884955592 & 0,503983169 & $-0,163754058$ & 0,848048096 & joint \\
\hline 40 & 63 & 287 & 0,471238898 & 1,8675023 & 0,434153274 & $-0,132733976$ & 0,891006524 & joint \\
\hline 40 & 28 & 239 & 1,082104136 & 1,029744259 & 0,756833805 & 0,454751628 & 0,469471563 & joint \\
\hline 40 & 54 & 303 & 0,628318531 & 2,14675498 & 0,492958191 & $-0,320130793$ & 0,809016994 & joint \\
\hline 40 & 86 & 43 & 0,06981317 & $-2,391101075$ & $-0,047573801$ & $-0,051016655$ & 0,99756405 & joint \\
\hline 40 & 60 & 277 & 0,523598776 & 1,692969374 & 0,496273076 & $-0,060934672$ & 0,866025404 & joint \\
\hline 40 & 62 & 279 & 0,488692191 & 1,727875959 & 0,463691589 & $-0,073441533$ & 0,882947593 & joint \\
\hline 40 & 61 & 283 & 0,506145483 & 1,79768913 & 0,472383981 & $-0,109058435$ & 0,874619707 & joint \\
\hline
\end{tabular}




\begin{tabular}{|c|c|c|c|c|c|c|c|c|}
\hline \multirow{2}{*}{ depth (meter) } & \multicolumn{2}{|c|}{ measures } & \multirow{2}{*}{$\theta$} & \multirow{2}{*}{$\phi$} & \multicolumn{3}{|c|}{ cosine director } & \multirow{2}{*}{$\begin{array}{l}\text { geological } \\
\text { structure }\end{array}$} \\
\hline & $\operatorname{dip}$ & dipdirection & & & $\mathbf{x}$ & $\mathbf{y}$ & $\mathbf{z}$ & \\
\hline 40 & 51 & 278 & 0,680678408 & 1,710422667 & 0,623195888 & $-0,08758447$ & 0,777145961 & joint \\
\hline 40 & 24 & 170 & 1,151917306 & $-0,174532925$ & $-0,158635504$ & 0,899666649 & 0,406736643 & joint \\
\hline 40 & 62 & 284 & 0,488692191 & 1,815142422 & 0,455526251 & $-0,11357545$ & 0,882947593 & joint \\
\hline 40 & 48 & 279 & 0,733038286 & 1,727875959 & 0,660892498 & $-0,104675088$ & 0,743144825 & joint \\
\hline 40 & 41 & 284 & 0,855211333 & 1,815142422 & 0,73229148 & $-0,182580772$ & 0,656059029 & joint \\
\hline 40 & 27 & 266 & 1,099557429 & 1,500983157 & 0,888836077 & 0,062153473 & 0,4539905 & joint \\
\hline 40 & 56 & 281 & 0,593411946 & 1,762782545 & 0,548918955 & $-0,106699036$ & 0,829037573 & joint \\
\hline 40 & 79 & 28 & 0,191986218 & $-2,652900463$ & $-0,089579397$ & $-0,168474343$ & 0,981627183 & joint \\
\hline 40 & 63 & 283 & 0,471238898 & 1,79768913 & 0,442354753 & $-0,102125642$ & 0,891006524 & joint \\
\hline 40 & 72 & 248 & 0,314159265 & 1,186823891 & 0,286515568 & 0,115759804 & 0,951056516 & joint \\
\hline 40 & 53 & 220 & 0,645771823 & 0,698131701 & 0,38683924 & 0,461017054 & 0,79863551 & joint \\
\hline 40 & 80 & 33 & 0,174532925 & $-2,565634$ & $-0,094575576$ & $-0,145633616$ & 0,984807753 & joint \\
\hline 40 & 50 & 289 & 0,698131701 & 1,902408885 & 0,607767625 & $-0,209271176$ & 0,766044443 & joint \\
\hline 40 & 52 & 278 & 0,663225116 & 1,710422667 & 0,6096699 & $-0,085683517$ & 0,788010754 & joint \\
\hline 40 & 56 & 262 & 0,593411946 & 1,431169987 & 0,553750877 & 0,07782461 & 0,829037573 & joint \\
\hline 40 & 59 & 258 & 0,541052068 & 1,361356817 & 0,503783257 & 0,107082437 & 0,857167301 & joint \\
\hline 40 & 50 & 271 & 0,698131701 & 1,588249619 & 0,64268971 & $-0,011218191$ & 0,766044443 & foliation \\
\hline 40 & 52 & 281 & 0,663225116 & 1,762782545 & 0,60435004 & $-0,117473748$ & 0,788010754 & foliation \\
\hline 40 & 89 & 204 & 0,017453293 & 0,41887902 & 0,007098533 & 0,015943567 & 0,999847695 & joint \\
\hline 40 & 50 & 271 & 0,698131701 & 1,588249619 & 0,64268971 & $-0,011218191$ & 0,766044443 & foliation \\
\hline 40 & 52 & 281 & 0,663225116 & 1,762782545 & 0,60435004 & $-0,117473748$ & 0,788010754 & foliation \\
\hline 40 & 80 & 33 & 0,174532925 & $-2,565634$ & $-0,094575576$ & \begin{tabular}{|l}
$-0,145633616$ \\
\end{tabular} & 0,984807753 & joint \\
\hline 40 & 89 & 204 & 0,017453293 & 0,41887902 & 0,007098533 & 0,015943567 & 0,999847695 & joint \\
\hline 40 & 44 & 271 & 0,802851456 & 1,588249619 & 0,719230241 & $-0,012554211$ & 0,69465837 & foliation \\
\hline 40 & 57 & 295 & 0,575958653 & 2,00712864 & 0,493610599 & $-0,230174402$ & 0,838670568 & foliation \\
\hline 40 & 78 & 60 & 0,20943951 & $-2,094395102$ & $-0,180056806$ & $-0,103955845$ & 0,978147601 & joint \\
\hline 40 & 40 & 291 & 0,872664626 & 1,93731547 & 0,715164098 & $-0,274525776$ & 0,64278761 & joint \\
\hline 40 & 88 & 340 & 0,034906585 & 2,792526803 & 0,011936331 & \begin{tabular}{|r|}
$-0,0327948$ \\
\end{tabular} & 0,999390827 & joint \\
\hline 40 & 49 & 331 & 0,715584993 & 2,635447171 & 0,318063729 & $-0,573802156$ & 0,75470958 & joint \\
\hline 40 & 27 & 236 & 1,099557429 & 0,977384381 & 0,738677886 & 0,498244525 & 0,4539905 & joint \\
\hline 40 & 81 & 120 & 0,157079633 & $-1,047197551$ & $-0,135476221$ & 0,078217233 & 0,987688341 & joint \\
\hline 40 & 72 & 238 & 0,314159265 & 1,012290966 & 0,262061274 & 0,163754058 & 0,951056516 & joint \\
\hline 40 & 30 & 174 & 1,047197551 & $-0,104719755$ & $-0,090524305$ & 0,861281226 & 0,5 & joint \\
\hline 40 & 60 & 225 & 0,523598776 & 0,785398163 & 0,353553391 & 0,353553391 & 0,866025404 & joint \\
\hline 40 & 61 & 250 & 0,506145483 & 1,221730476 & 0,455572023 & 0,165814656 & 0,874619707 & joint \\
\hline 40 & 60 & 284 & 0,523598776 & 1,815142422 & 0,485147863 & $-0,120960948$ & 0,866025404 & joint \\
\hline 40 & 32 & 241 & 1,012290966 & 1,064650844 & 0,741719578 & 0,411141875 & 0,529919264 & joint \\
\hline 40 & 71 & 234 & 0,331612558 & 0,942477796 & 0,26339017 & 0,19136416 & 0,945518576 & joint \\
\hline 40 & 71 & 239 & 0,331612558 & 1,029744259 & 0,279066376 & 0,167679996 & 0,945518576 & joint \\
\hline 40 & 72 & 236 & 0,314159265 & 0,977384381 & 0,256186699 & 0,17280011 & 0,951056516 & joint \\
\hline 40 & 24 & 300 & 1,151917306 & 2,094395102 & 0,791153574 & $-0,456772729$ & 0,406736643 & joint \\
\hline 40 & 80 & 288 & 0,174532925 & 1,884955592 & 0,165149231 & $-0,053660238$ & 0,984807753 & joint \\
\hline 40 & 28 & 255 & 1,082104136 & 1,308996939 & 0,852861883 & 0,228523653 & 0,469471563 & joint \\
\hline 40 & 49 & 283 & 0,715584993 & 1,79768913 & 0,639244279 & $-0,14758117$ & 0,75470958 & joint \\
\hline 40 & 70 & 226 & 0,34906585 & 0,802851456 & 0,246028702 & 0,237587155 & 0,939692621 & joint \\
\hline 40 & 49 & 281 & 0,715584993 & 1,762782545 & 0,644005377 & $-0,125181964$ & 0,75470958 & joint \\
\hline 40 & 67 & 177 & 0,401425728 & $-0,052359878$ & $-0,020449287$ & 0,390195645 & 0,920504853 & joint \\
\hline 40 & 57 & 277 & 0,575958653 & 1,692969374 & 0,540579378 & $-0,066374802$ & 0,838670568 & joint \\
\hline 40 & 52 & 218 & 0,663225116 & 0,663225116 & 0,379039052 & 0,485147863 & 0,788010754 & joint \\
\hline 40 & 60 & 286 & 0,523598776 & 1,850049007 & 0,480630848 & $-0,137818678$ & 0,866025404 & joint \\
\hline 40 & 52 & 273 & 0,663225116 & 1,623156204 & 0,614817733 & $-0,032221232$ & 0,788010754 & joint \\
\hline 40 & 48 & 289 & 0,733038286 & 1,902408885 & 0,632675418 & $-0,217847617$ & 0,743144825 & joint \\
\hline 40 & 63 & 3 & 0,471238898 & $-3,089232776$ & $-0,023760027$ & $-0,453368322$ & 0,891006524 & joint \\
\hline 40 & 25 & 298 & 1,134464014 & 2,059488517 & 0,800222279 & $-0,425485733$ & 0,422618262 & joint \\
\hline 40 & 88 & 215 & 0,034906585 & 0,610865238 & 0,020017529 & 0,028587994 & 0,999390827 & joint \\
\hline 40 & 88 & 188 & 0,034906585 & 0,13962634 & 0,004857071 & 0,034559857 & 0,999390827 & joint \\
\hline 40 & 40 & 273 & 0,872664626 & 1,623156204 & 0,764994606 & $-0,040091668$ & 0,64278761 & foliation \\
\hline
\end{tabular}




\begin{tabular}{|c|c|c|c|c|c|c|c|c|}
\hline \multirow{2}{*}{ depth (meter) } & \multicolumn{2}{|c|}{ measures } & \multirow{2}{*}{$\theta$} & \multirow{2}{*}{$\phi$} & \multicolumn{3}{|c|}{ cosine director } & \multirow{2}{*}{$\begin{array}{l}\text { geological } \\
\text { structure }\end{array}$} \\
\hline & $\operatorname{dip}$ & dipdirection & & & $\mathbf{x}$ & $\mathbf{y}$ & $\mathbf{z}$ & \\
\hline 40 & 35 & 265 & 0,959931089 & 1,483529864 & 0,816034923 & 0,071393805 & 0,573576436 & foliation \\
\hline 40 & 41 & 272 & 0,855211333 & 1,605702912 & 0,754249832 & $-0,026338985$ & 0,656059029 & foliation \\
\hline 40 & 79 & 343 & 0,191986218 & 2,844886681 & 0,055787151 & $-0,18247155$ & 0,981627183 & joint \\
\hline 40 & 87 & 201 & 0,052359878 & 0,366519143 & 0,018755529 & 0,048859824 & 0,998629535 & joint \\
\hline 40 & 40 & 273 & 0,872664626 & 1,623156204 & 0,764994606 & $-0,040091668$ & 0,64278761 & foliation \\
\hline 40 & 41 & 272 & 0,855211333 & 1,605702912 & 0,754249832 & $-0,026338985$ & 0,656059029 & foliation \\
\hline 40 & 79 & 343 & 0,191986218 & 2,844886681 & 0,055787151 & $-0,18247155$ & 0,981627183 & joint \\
\hline 40 & 35 & 265 & 0,959931089 & 1,483529864 & 0,816034923 & 0,071393805 & 0,573576436 & joint \\
\hline 40 & 87 & 201 & 0,052359878 & 0,366519143 & 0,018755529 & 0,048859824 & 0,998629535 & joint \\
\hline 40 & 50 & 283 & 0,698131701 & 1,79768913 & 0,626313005 & $-0,144595751$ & 0,766044443 & foliation \\
\hline 40 & 83 & 38 & 0,122173048 & $-2,478367538$ & $-0,07503026$ & $-0,096034353$ & 0,992546152 & joint \\
\hline 40 & 77 & 46 & 0,226892803 & $-2,338741198$ & $-0,161816247$ & $-0,156264133$ & 0,974370065 & joint \\
\hline 40 & 80 & 35 & 0,174532925 & $-2,530727415$ & $-0,099600503$ & $-0,14224426$ & 0,984807753 & joint \\
\hline 40 & 87 & 214 & 0,052359878 & 0,593411946 & 0,029265895 & 0,043388474 & 0,998629535 & joint \\
\hline 40 & 87 & 45 & 0,052359878 & $-2,35619449$ & $-0,03700711$ & $-0,03700711$ & 0,998629535 & joint \\
\hline 40 & 68 & 40 & 0,383972435 & $-2,443460953$ & $-0,240792477$ & $-0,286965299$ & 0,927183855 & joint \\
\hline 40 & 70 & 45 & 0,34906585 & $-2,35619449$ & $-0,241844763$ & $-0,241844763$ & 0,939692621 & joint \\
\hline 40 & 87 & 238 & 0,052359878 & 1,012290966 & 0,044383408 & 0,027733831 & 0,998629535 & joint \\
\hline 40 & 49 & 69 & 0,715584993 & $-1,93731547$ & $-0,612483868$ & $-0,235110529$ & 0,75470958 & joint \\
\hline 40 & 46 & 315 & 0,767944871 & 2,35619449 & 0,491197644 & $-0,491197644$ & 0,7193398 & joint \\
\hline 40 & 48 & 287 & 0,733038286 & 1,8675023 & 0,639892781 & $-0,195634856$ & 0,743144825 & joint \\
\hline 40 & 52 & 293 & 0,663225116 & 1,972222055 & 0,566719376 & $-0,240558103$ & 0,788010754 & joint \\
\hline 40 & 87 & 220 & 0,052359878 & 0,698131701 & 0,033640904 & 0,040091668 & 0,998629535 & joint \\
\hline 40 & 85 & 218 & 0,087266463 & 0,663225116 & 0,053658433 & 0,068679663 & 0,996194698 & joint \\
\hline 40 & 64 & 241 & 0,453785606 & 1,064650844 & 0,383408044 & 0,212526549 & 0,898794046 & joint \\
\hline 40 & 62 & 287 & 0,488692191 & 1,8675023 & 0,448957888 & $-0,137260201$ & 0,882947593 & joint \\
\hline 40 & 53 & 296 & 0,645771823 & 2,024581932 & 0,54090776 & $-0,263818342$ & 0,79863551 & joint \\
\hline 40 & 56 & 282 & 0,593411946 & 1,780235837 & 0,546973197 & $-0,116262742$ & 0,829037573 & joint \\
\hline 40 & 86 & 31 & 0,06981317 & $-2,600540585$ & $-0,03592724$ & $-0,059792968$ & 0,99756405 & joint \\
\hline 40 & 49 & 219 & 0,715584993 & 0,680678408 & 0,412871325 & 0,509853625 & 0,75470958 & joint \\
\hline 40 & 69 & 188 & 0,366519143 & 0,13962634 & 0,049875179 & 0,354880337 & 0,933580426 & joint \\
\hline 40 & 86 & 35 & 0,06981317 & $-2,530727415$ & $-0,04001067$ & $-0,057141158$ & 0,99756405 & joint \\
\hline 40 & 48 & 271 & 0,733038286 & 1,588249619 & 0,669028695 & $-0,011677939$ & 0,743144825 & joint \\
\hline 40 & 74 & 47 & 0,27925268 & $-2,321287905$ & $-0,2015884$ & $-0,187984225$ & 0,961261696 & joint \\
\hline 40 & 78 & 233 & 0,20943951 & 0,925024504 & 0,166045659 & 0,125124379 & 0,978147601 & joint \\
\hline 40 & 40 & 264 & 0,872664626 & 1,466076572 & 0,761847972 & 0,080073448 & 0,64278761 & joint \\
\hline 40 & 84 & 348 & 0,104719755 & 2,932153143 & 0,02173269 & $-0,102244266$ & 0,994521895 & joint \\
\hline 40 & 55 & 281 & 0,610865238 & 1,762782545 & 0,563038222 & $-0,109443544$ & 0,819152044 & joint \\
\hline 40 & 87 & 199 & 0,052359878 & 0,331612558 & 0,017038921 & 0,049484619 & 0,998629535 & joint \\
\hline 40 & 52 & 278 & 0,663225116 & 1,710422667 & 0,6096699 & $-0,085683517$ & 0,788010754 & joint \\
\hline 40 & 86 & 215 & 0,06981317 & 0,610865238 & 0,04001067 & 0,057141158 & 0,99756405 & joint \\
\hline 40 & 53 & 278 & 0,645771823 & 1,710422667 & 0,595958201 & $-0,083756463$ & 0,79863551 & joint \\
\hline 40 & 78 & 288 & 0,20943951 & 1,884955592 & 0,197735768 & $-0,064248246$ & 0,978147601 & foliation \\
\hline 40 & 65 & 352 & 0,436332313 & 3,001966313 & 0,058817094 & $-0,41850537$ & 0,906307787 & joint \\
\hline 40 & 65 & 45 & 0,436332313 & $-2,35619449$ & $-0,298836239$ & $-0,298836239$ & 0,906307787 & joint \\
\hline 40 & 78 & 288 & 0,20943951 & 1,884955592 & 0,197735768 & $-0,064248246$ & 0,978147601 & foliation \\
\hline 40 & 65 & 45 & 0,436332313 & $-2,35619449$ & $-0,298836239$ & $-0,298836239$ & 0,906307787 & joint \\
\hline 40 & 58 & 29 & 0,558505361 & $-2,635447171$ & $-0,256909957$ & $-0,463477832$ & 0,848048096 & joint \\
\hline 40 & 79 & 208 & 0,191986218 & 0,488692191 & 0,089579397 & 0,168474343 & 0,981627183 & joint \\
\hline 40 & 68 & 232 & 0,383972435 & 0,907571211 & 0,295194024 & 0,230630848 & 0,927183855 & joint \\
\hline 40 & 54 & 269 & 0,628318531 & 1,553343034 & 0,58769573 & 0,010258267 & 0,809016994 & foliation \\
\hline 40 & 55 & 275 & 0,610865238 & 1,658062789 & 0,571393805 & $-0,04999048$ & 0,819152044 & foliation \\
\hline 40 & 58 & 280 & 0,558505361 & 1,745329252 & 0,5218686 & $-0,092019515$ & 0,848048096 & foliation \\
\hline 40 & 19 & 251 & 1,239183769 & 1,239183769 & 0,894005377 & 0,307830738 & 0,325568154 & joint \\
\hline 40 & 88 & 49 & 0,034906585 & $-2,28638132$ & $-0,026338985$ & $-0,02289613$ & 0,999390827 & joint \\
\hline 40 & 85 & 276 & 0,087266463 & 1,675516082 & 0,086678294 & $-0,009110256$ & 0,996194698 & joint \\
\hline 40 & 58 & 286 & 0,558505361 & 1,850049007 & 0,509391091 & $-0,146065545$ & 0,848048096 & joint \\
\hline
\end{tabular}




\begin{tabular}{|c|c|c|c|c|c|c|c|c|}
\hline \multirow{2}{*}{ depth (meter) } & \multicolumn{2}{|c|}{ measures } & \multirow{2}{*}{$\theta$} & \multirow{2}{*}{$\phi$} & \multicolumn{3}{|c|}{ cosine director } & \multirow{2}{*}{$\begin{array}{l}\text { geological } \\
\text { structure }\end{array}$} \\
\hline & $\operatorname{dip}$ & dipdirection & & & $\mathbf{x}$ & $\mathbf{y}$ & $\mathbf{z}$ & \\
\hline 40 & 59 & 277 & 0,541052068 & 1,692969374 & 0,511199059 & $-0,062767352$ & 0,857167301 & joint \\
\hline 40 & 55 & 296 & 0,610865238 & 2,024581932 & 0,515527086 & $-0,25143936$ & 0,819152044 & joint \\
\hline 40 & 50 & 266 & 0,698131701 & 1,500983157 & 0,641221811 & 0,044838597 & 0,766044443 & joint \\
\hline 40 & 52 & 289 & 0,663225116 & 1,902408885 & 0,582119361 & $-0,20043977$ & 0,788010754 & joint \\
\hline 40 & 26 & 270 & 1,117010721 & 1,570796327 & 0,898794046 & $5,50578 \mathrm{E}-17$ & 0,438371147 & joint \\
\hline 40 & 65 & 342 & 0,436332313 & 2,827433388 & 0,130596225 & $-0,401933852$ & 0,906307787 & joint \\
\hline 40 & 28 & 238 & 1,082104136 & 1,012290966 & 0,748782025 & 0,467890939 & 0,469471563 & joint \\
\hline 40 & 25 & 239 & 1,134464014 & 1,029744259 & 0,776857399 & 0,466783018 & 0,422618262 & joint \\
\hline 40 & 87 & 329 & 0,052359878 & 2,600540585 & 0,02695501 & $-0,04486067$ & 0,998629535 & joint \\
\hline 40 & 40 & 264 & 0,872664626 & 1,466076572 & 0,761847972 & 0,080073448 & 0,64278761 & joint \\
\hline 40 & 56 & 285 & 0,593411946 & 1,832595715 & 0,540138867 & $-0,144729773$ & 0,829037573 & joint \\
\hline 40 & 87 & 211 & 0,052359878 & 0,541052068 & 0,02695501 & 0,04486067 & 0,998629535 & joint \\
\hline $41-50$ & 50 & 247 & 0,698131701 & 1,169370599 & 0,591689114 & 0,251157128 & 0,766044443 & foliation \\
\hline 50 & 50 & 247 & 0,698131701 & 1,169370599 & 0,591689114 & 0,251157128 & 0,766044443 & foliation \\
\hline 50 & 49 & 272 & 0,715584993 & 1,605702912 & 0,655659376 & $-0,02289613$ & 0,75470958 & foliation \\
\hline 50 & 74 & 240 & 0,27925268 & 1,047197551 & 0,238708952 & 0,137818678 & 0,961261696 & joint \\
\hline 50 & 59 & 278 & 0,541052068 & 1,710422667 & 0,51002576 & $-0,071679446$ & 0,857167301 & joint \\
\hline 50 & 52 & 305 & 0,663225116 & 2,181661565 & 0,504320356 & $-0,353128915$ & 0,788010754 & joint \\
\hline 50 & 48 & 261 & 0,733038286 & 1,413716694 & 0,660892498 & 0,104675088 & 0,743144825 & joint \\
\hline 50 & 66 & 164 & 0,41887902 & $-0,27925268$ & $-0,112111813$ & 0,390980355 & 0,913545458 & joint \\
\hline 50 & 52 & 281 & 0,663225116 & 1,762782545 & 0,60435004 & $-0,117473748$ & 0,788010754 & joint \\
\hline 50 & 56 & 51 & 0,593411946 & $-2,251474735$ & $-0,434574507$ & $-0,351911497$ & 0,829037573 & joint \\
\hline 50 & 58 & 265 & 0,558505361 & 1,483529864 & 0,527902761 & 0,046185507 & 0,848048096 & foliation \\
\hline 50 & 36 & 272 & 0,942477796 & 1,605702912 & 0,808524163 & $-0,028234286$ & 0,587785252 & joint \\
\hline 50 & 82 & 42 & 0,13962634 & $-2,408554368$ & $-0,093124981$ & $-0,10342577$ & 0,990268069 & joint \\
\hline 50 & 80 & 171 & 0,174532925 & $-0,157079633$ & $-0,02716456$ & 0,17151028 & 0,984807753 & joint \\
\hline 50 & 81 & 165 & 0,157079633 & $-0,261799388$ & $-0,040488219$ & 0,15110409 & 0,987688341 & joint \\
\hline 50 & 79 & 165 & 0,191986218 & $-0,261799388$ & $-0,049385002$ & 0,184307337 & 0,981627183 & joint \\
\hline 50 & 35 & 112 & 0,959931089 & $-1,186823891$ & $-0,75950455$ & 0,306859757 & 0,573576436 & joint \\
\hline 50 & 88 & 181 & 0,034906585 & 0,017453293 & 0,00060908 & 0,034894181 & 0,999390827 & joint \\
\hline 50 & 89 & 185 & 0,017453293 & 0,087266463 & 0,001521077 & 0,017385995 & 0,999847695 & joint \\
\hline 50 & 20 & 221 & 1,221730476 & 0,715584993 & 0,616493828 & 0,709195023 & 0,342020143 & joint \\
\hline 50 & 86 & 184 & 0,06981317 & 0,06981317 & 0,004865966 & 0,06958655 & 0,99756405 & joint \\
\hline 50 & 70 & 234 & 0,34906585 & 0,942477796 & 0,276700108 & 0,201034396 & 0,939692621 & joint \\
\hline 50 & 88 & 175 & 0,034906585 & $-0,087266463$ & $-0,003041692$ & 0,034766694 & 0,999390827 & joint \\
\hline 50 & 39 & 273 & 0,890117919 & 1,623156204 & 0,77608091 & $-0,040672677$ & 0,629320391 & joint \\
\hline 50 & 59 & 344 & 0,541052068 & 2,862339973 & 0,141963733 & $-0,495086373$ & 0,857167301 & joint \\
\hline 50 & 38 & 251 & 0,907571211 & 1,239183769 & 0,745078805 & 0,256551207 & 0,615661475 & joint \\
\hline 50 & 70 & 241 & 0,34906585 & 1,064650844 & 0,299137558 & 0,165814656 & 0,939692621 & joint \\
\hline 50 & 40 & 229 & 0,872664626 & 0,855211333 & 0,57814108 & 0,502570374 & 0,64278761 & joint \\
\hline 50 & 88 & 225 & 0,034906585 & 0,785398163 & 0,024677671 & 0,024677671 & 0,999390827 & joint \\
\hline 50 & 50 & 290 & 0,698131701 & 1,919862177 & 0,604022774 & $-0,21984631$ & 0,766044443 & foliation \\
\hline 50 & 43 & 264 & 0,820304748 & 1,466076572 & 0,72734727 & 0,076447279 & 0,68199836 & foliation \\
\hline 50 & 53 & 280 & 0,645771823 & 1,745329252 & 0,592672101 & $-0,104504082$ & 0,79863551 & foliation \\
\hline 50 & 74 & 250 & 0,27925268 & 1,221730476 & 0,259014389 & 0,094273528 & 0,961261696 & foliation \\
\hline 50 & 60 & 260 & 0,523598776 & 1,396263402 & 0,492403877 & 0,086824089 & 0,866025404 & foliation \\
\hline 50 & 40 & 285 & 0,872664626 & 1,832595715 & 0,739942112 & $-0,198266891$ & 0,64278761 & foliation \\
\hline 50 & 68 & 280 & 0,383972435 & 1,745329252 & 0,368915478 & $-0,065049752$ & 0,927183855 & foliation \\
\hline 50 & 45 & 288 & 0,785398163 & 1,884955592 & 0,672498512 & $-0,218508012$ & 0,707106781 & foliation \\
\hline 50 & 41 & 276 & 0,855211333 & 1,675516082 & 0,750575202 & $-0,078888633$ & 0,656059029 & joint \\
\hline 50 & 78 & 358 & 0,20943951 & 3,106686069 & 0,007256013 & $-0,207785037$ & 0,978147601 & joint \\
\hline 50 & 78 & 153 & 0,20943951 & $-0,471238898$ & $-0,094389932$ & 0,185250673 & 0,978147601 & joint \\
\hline 50 & 85 & 347 & 0,087266463 & 2,914699851 & 0,019605776 & $-0,084921947$ & 0,996194698 & joint \\
\hline 50 & 75 & 58 & 0,261799388 & $-2,129301687$ & $-0,219490998$ & $-0,137153198$ & 0,965925826 & joint \\
\hline 50 & 70 & 20 & 0,34906585 & $-2,792526803$ & $-0,116977778$ & $-0,321393805$ & 0,939692621 & joint \\
\hline 50 & 75 & 218 & 0,261799388 & 0,663225116 & 0,159344915 & 0,203952191 & 0,965925826 & joint \\
\hline 50 & 80 & 211 & 0,174532925 & 0,541052068 & 0,089435423 & 0,14884554 & 0,984807753 & joint \\
\hline
\end{tabular}




\begin{tabular}{|c|c|c|c|c|c|c|c|c|}
\hline \multirow{2}{*}{ depth (meter) } & \multicolumn{2}{|c|}{ measures } & \multirow{2}{*}{$\theta$} & \multirow{2}{*}{$\phi$} & \multicolumn{3}{|c|}{ cosine director } & \multirow{2}{*}{$\begin{array}{l}\text { geological } \\
\text { structure }\end{array}$} \\
\hline & $\operatorname{dip}$ & dipdirection & & & $\mathbf{x}$ & $\mathbf{y}$ & $\mathbf{z}$ & \\
\hline 50 & 55 & 292 & 0,610865238 & 1,954768762 & 0,531810811 & $-0,214865515$ & 0,819152044 & joint \\
\hline 50 & 58 & 8 & 0,558505361 & $-3,001966313$ & $-0,073750507$ & $-0,524762126$ & 0,848048096 & joint \\
\hline 50 & 82 & 48 & 0,13962634 & $-2,303834613$ & $-0,10342577$ & $-0,093124981$ & 0,990268069 & joint \\
\hline 50 & 76 & 301 & 0,244346095 & 2,111848395 & 0,207367538 & $-0,124598987$ & 0,970295726 & joint \\
\hline 50 & 79 & 225 & 0,191986218 & 0,785398163 & 0,134922335 & 0,134922335 & 0,981627183 & joint \\
\hline 50 & 81 & 43 & 0,157079633 & $-2,391101075$ & $-0,106688049$ & $-0,114408925$ & 0,987688341 & joint \\
\hline 50 & 80 & 158 & 0,174532925 & $-0,383972435$ & $-0,065049752$ & 0,161003787 & 0,984807753 & joint \\
\hline 50 & 69 & 53 & 0,366519143 & $-2,21656815$ & $-0,28620537$ & $-0,215671216$ & 0,933580426 & joint \\
\hline 50 & 84 & 43 & 0,104719755 & $-2,391101075$ & $-0,071288241$ & $-0,076447279$ & 0,994521895 & joint \\
\hline 50 & 70 & 220 & 0,34906585 & 0,698131701 & \begin{tabular}{|l|}
0,21984631 \\
\end{tabular} & \begin{tabular}{|c|}
0,26200263 \\
\end{tabular} & 0,939692621 & joint \\
\hline 50 & 72 & 108 & 0,314159265 & $-1,256637061$ & $-0,293892626$ & 0,095491503 & 0,951056516 & joint \\
\hline 50 & 82 & 52 & 0,13962634 & $-2,234021443$ & $-0,1096699$ & $-0,085683517$ & 0,990268069 & joint \\
\hline 50 & 72 & 108 & 0,314159265 & $-1,256637061$ & $-0,293892626$ & 0,095491503 & 0,951056516 & joint \\
\hline 50 & 86 & 220 & 0,06981317 & 0,698131701 & 0,044838597 & 0,053436559 & 0,99756405 & joint \\
\hline 50 & 72 & 8 & 0,314159265 & $-3,001966313$ & $-0,043006853$ & $-0,306009662$ & 0,951056516 & joint \\
\hline 50 & 72 & 37 & 0,314159265 & $-2,49582083$ & $-0,18597107$ & $-0,246791945$ & 0,951056516 & joint \\
\hline 50 & 79 & 228 & 0,191986218 & 0,837758041 & 0,141798718 & 0,127676139 & 0,981627183 & joint \\
\hline 50 & 87 & 351 & 0,052359878 & 2,984513021 & 0,008187147 & $-0,051691614$ & 0,998629535 & joint \\
\hline 50 & 18 & 310 & 1,256637061 & 2,268928028 & 0,728551559 & $-0,611327345$ & 0,309016994 & joint \\
\hline 50 & 55 & 228 & 0,610865238 & 0,837758041 & 0,426250361 & 0,383797549 & 0,819152044 & joint \\
\hline 50 & 84 & 353 & 0,104719755 & 3,019419606 & 0,012738815 & $-0,103749324$ & 0,994521895 & joint \\
\hline 50 & 55 & 228 & 0,610865238 & 0,837758041 & 0,426250361 & 0,383797549 & 0,819152044 & joint \\
\hline 50 & 35 & 321 & 0,959931089 & 2,460914245 & 0,515509085 & $-0,636600703$ & 0,573576436 & joint \\
\hline 50 & 33 & 269 & 0,994837674 & 1,553343034 & 0,838542834 & 0,01463682 & 0,544639035 & foliation \\
\hline 50 & 80 & 264 & 0,174532925 & 1,466076572 & 0,172696915 & 0,018151177 & 0,984807753 & foliation \\
\hline 50 & 57 & 265 & 0,575958653 & 1,483529864 & 0,542566519 & 0,04746842 & 0,838670568 & foliation \\
\hline 50 & 78 & 48 & 0,20943951 & $-2,303834613$ & $-0,154508497$ & $-0,139120076$ & 0,978147601 & joint \\
\hline 50 & 79 & 218 & 0,191986218 & 0,663225116 & 0,117473748 & \begin{tabular}{|l}
0,15035954 \\
\end{tabular} & 0,981627183 & joint \\
\hline 50 & 80 & 224 & 0,174532925 & 0,767944871 & 0,12062616 & 0,124912045 & 0,984807753 & joint \\
\hline 50 & 77 & 359 & 0,226892803 & 3,124139361 & 0,003925937 & $-0,224916793$ & 0,974370065 & joint \\
\hline 50 & 67 & 5 & 0,401425728 & $-3,054326191$ & $-0,034054462$ & $-0,389244279$ & 0,920504853 & joint \\
\hline 50 & 51 & 255 & 0,680678408 & 1,308996939 & 0,607876819 & 0,162880103 & 0,777145961 & foliation \\
\hline 50 & 88 & 227 & 0,034906585 & 0,820304748 & 0,025523876 & 0,0238014 & 0,999390827 & joint \\
\hline 50 & 62 & 110 & 0,488692191 & $-1,221730476$ & $-0,441158963$ & 0,160568731 & 0,882947593 & joint \\
\hline 50 & 68 & 43 & 0,383972435 & $-2,391101075$ & $-0,255481082$ & $-0,273969919$ & 0,927183855 & joint \\
\hline 50 & 44 & 273 & 0,802851456 & 1,623156204 & 0,71835397 & $-0,037647336$ & 0,69465837 & foliation \\
\hline 50 & 27 & 275 & 1,099557429 & 1,658062789 & 0,887615975 & $-0,077656335$ & 0,4539905 & foliation \\
\hline 50 & 74 & 240 & 0,27925268 & 1,047197551 & 0,238708952 & 0,137818678 & 0,961261696 & joint \\
\hline 50 & 62 & 270 & 0,488692191 & 1,570796327 & 0,469471563 & $2,87586 \mathrm{E}-17$ & 0,882947593 & foliation \\
\hline 50 & 59 & 273 & 0,541052068 & 1,623156204 & 0,514332233 & $-0,02695501$ & 0,857167301 & foliation \\
\hline 50 & 85 & 283 & 0,087266463 & 1,79768913 & 0,084921947 & $-0,019605776$ & 0,996194698 & foliation \\
\hline 50 & 52 & 277 & 0,663225116 & 1,692969374 & 0,611072428 & $-0,07503026$ & 0,788010754 & foliation \\
\hline 50 & 76 & 226 & 0,244346095 & 0,802851456 & 0,174024048 & 0,16805307 & 0,970295726 & joint \\
\hline 50 & 29 & 122 & 1,064650844 & $-1,012290966$ & $-0,741719578$ & 0,463477832 & 0,48480962 & joint \\
\hline 50 & 27 & 114 & 1,099557429 & $-1,151917306$ & $-0,813974963$ & 0,362405003 & 0,4539905 & joint \\
\hline 50 & 87 & 178 & 0,052359878 & $-0,034906585$ & $-0,001826499$ & 0,052304075 & 0,998629535 & joint \\
\hline 50 & 61 & 288 & 0,506145483 & 1,884955592 & 0,461081348 & $-0,149814412$ & 0,874619707 & joint \\
\hline 50 & 82 & 243 & 0,13962634 & 1,099557429 & 0,124004141 & 0,063183266 & 0,990268069 & joint \\
\hline 50 & 44 & 110 & 0,802851456 & $-1,221730476$ & $-0,675958302$ & 0,246028702 & 0,69465837 & joint \\
\hline 50 & 52 & 272 & 0,663225116 & 1,605702912 & 0,615286431 & $-0,021486276$ & 0,788010754 & joint \\
\hline 50 & 78 & 18 & 0,20943951 & $-2,827433388$ & $-0,064248246$ & $-0,197735768$ & 0,978147601 & joint \\
\hline 50 & 53 & 291 & 0,645771823 & 1,93731547 & 0,561842726 & $-0,215671216$ & 0,79863551 & joint \\
\hline 50 & 84 & 311 & 0,104719755 & 2,28638132 & 0,078888633 & $-0,068576842$ & 0,994521895 & joint \\
\hline 50 & 83 & 316 & 0,122173048 & 2,373647783 & 0,084657559 & $-0,087665469$ & 0,992546152 & joint \\
\hline 50 & 87 & 225 & 0,052359878 & 0,785398163 & 0,03700711 & 0,03700711 & 0,998629535 & joint \\
\hline 50 & 88 & 221 & 0,034906585 & 0,715584993 & 0,02289613 & 0,026338985 & 0,999390827 & joint \\
\hline 50 & 55 & 278 & 0,610865238 & 1,710422667 & 0,56799443 & $-0,079826411$ & 0,819152044 & joint \\
\hline
\end{tabular}




\begin{tabular}{|c|c|c|c|c|c|c|c|c|}
\hline \multirow{2}{*}{ depth (meter) } & \multicolumn{2}{|c|}{ measures } & \multirow{2}{*}{$\boldsymbol{\theta}$} & \multirow{2}{*}{$\phi$} & \multicolumn{3}{|c|}{ cosine director } & \multirow{2}{*}{$\begin{array}{l}\text { geological } \\
\text { structure }\end{array}$} \\
\hline & $\operatorname{dip}$ & dipdirection & & & $\mathbf{x}$ & $\mathbf{y}$ & $\mathbf{z}$ & \\
\hline 50 & 57 & 290 & 0,575958653 & 1,919862177 & 0,511793282 & $-0,186277521$ & 0,838670568 & joint \\
\hline 50 & 89 & 25 & 0,017453293 & $-2,705260341$ & $-0,007375706$ & $-0,015817252$ & 0,999847695 & joint \\
\hline 50 & 67 & 271 & 0,401425728 & 1,588249619 & 0,390671618 & $-0,006819198$ & 0,920504853 & joint \\
\hline 50 & 30 & 268 & 1,047197551 & 1,535889742 & 0,865497845 & 0,030223851 & 0,5 & foliation \\
\hline 50 & 48 & 283 & 0,733038286 & 1,79768913 & 0,651980832 & $-0,150521635$ & 0,743144825 & foliation \\
\hline 50 & 32 & 244 & 1,012290966 & 1,117010721 & 0,76222058 & 0,371759816 & 0,529919264 & foliation \\
\hline 50 & 49 & 278 & 0,715584993 & 1,710422667 & 0,649674308 & $-0,091305769$ & 0,75470958 & foliation \\
\hline 50 & 83 & 215 & 0,122173048 & 0,610865238 & 0,069901384 & 0,099829522 & 0,992546152 & joint \\
\hline 50 & 83 & 139 & 0,122173048 & $-0,715584993$ & $-0,079953483$ & 0,091975961 & 0,992546152 & joint \\
\hline 50 & 80 & 300 & 0,174532925 & 2,094395102 & 0,150383733 & $-0,086824089$ & 0,984807753 & joint \\
\hline 50 & 36 & 272 & 0,942477796 & 1,605702912 & 0,808524163 & $-0,028234286$ & 0,587785252 & joint \\
\hline 50 & 85 & 184 & 0,087266463 & 0,06981317 & 0,006079677 & 0,086943436 & 0,996194698 & joint \\
\hline 50 & 88 & 186 & 0,034906585 & 0,104719755 & 0,003647991 & 0,034708314 & 0,999390827 & joint \\
\hline 50 & 75 & 174 & 0,261799388 & $-0,104719755$ & $-0,027053957$ & 0,257401207 & 0,965925826 & joint \\
\hline 50 & 88 & 182 & 0,034906585 & 0,034906585 & 0,001217975 & 0,034878237 & 0,999390827 & joint \\
\hline 50 & 41 & 268 & 0,855211333 & 1,535889742 & 0,754249832 & 0,026338985 & 0,656059029 & foliation \\
\hline 50 & 31 & 202 & 1,029744259 & 0,383972435 & 0,321100523 & 0,794751682 & 0,515038075 & joint \\
\hline 50 & 43 & 157 & 0,820304748 & $-0,401425728$ & $-0,285762657$ & 0,673214632 & 0,68199836 & joint \\
\hline 50 & 30 & 241 & 1,047197551 & 1,064650844 & 0,757442885 & 0,419857447 & 0,5 & joint \\
\hline 50 & 25 & 210 & 1,134464014 & 0,523598776 & 0,453153894 & 0,7848855667 & 0,422618262 & joint \\
\hline 50 & 87 & 127 & 0,052359878 & $-0,925024504$ & $-0,041797353$ & 0,031496565 & 0,998629535 & joint \\
\hline 50 & 29 & 245 & 1,064650844 & 1,134464014 & 0,792674651 & 0,36963026 & 0,48480962 & joint \\
\hline 50 & 78 & 335 & 0,20943951 & 2,705260341 & 0,087867277 & $-0,188431984$ & 0,978147601 & joint \\
\hline 50 & 67 & 222 & 0,401425728 & 0,733038286 & 0,261450157 & 0,290369816 & 0,920504853 & joint \\
\hline 50 & 88 & 130 & 0,034906585 & $-0,872664626$ & $-0,026734566$ & 0,022432964 & 0,999390827 & joint \\
\hline 50 & 38 & 161 & 0,907571211 & $-0,331612558$ & $-0,256551207$ & 0,745078805 & 0,615661475 & joint \\
\hline 50 & 49 & 258 & 0,715584993 & 1,361356817 & 0,641722565 & 0,136402342 & 0,75470958 & foliation \\
\hline 50 & 52 & 261 & 0,663225116 & 1,413716694 & 0,608081661 & 0,096310674 & 0,788010754 & foliation \\
\hline 50 & 50 & 284 & 0,698131701 & 1,815142422 & 0,623694071 & $-0,155504397$ & 0,766044443 & foliation \\
\hline 50 & 42 & 28 & 0,837758041 & $-2,652900463$ & $-0,348885363$ & $-0,656157935$ & 0,669130606 & foliation \\
\hline 50 & 53 & 275 & 0,645771823 & 1,658062789 & 0,599524935 & $-0,052451635$ & 0,79863551 & foliation \\
\hline 50 & 47 & 286 & 0,750491578 & 1,850049007 & 0,6555789 & $-0,187984225$ & 0,731353702 & foliation \\
\hline 50 & 52 & 291 & 0,663225116 & 1,93731547 & 0,574769503 & $-0,220633341$ & 0,788010754 & foliation \\
\hline 50 & 44 & 264 & 0,802851456 & 1,466076572 & 0,715399182 & 0,075191484 & 0,69465837 & foliation \\
\hline 50 & 47 & 275 & 0,750491578 & 1,658062789 & 0,67940315 & $-0,059440074$ & 0,731353702 & foliation \\
\hline 50 & 83 & 291 & 0,122173048 & 1,93731547 & 0,113774834 & $-0,043674067$ & 0,992546152 & foliation \\
\hline 50 & 74 & 68 & 0,27925268 & $-1,954768762$ & $-0,255566506$ & $-0,103255571$ & 0,961261696 & foliation \\
\hline 50 & 87 & 11 & 0,052359878 & $-2,949606436$ & $-0,009986171$ & $-0,051374397$ & 0,998629535 & foliation \\
\hline 50 & 84 & 234 & 0,104719755 & 0,942477796 & 0,084565303 & 0,061440289 & 0,994521895 & foliation \\
\hline 50 & 89 & 175 & 0,017453293 & $-0,087266463$ & $-0,001521077$ & 0,017385995 & 0,999847695 & foliation \\
\hline 50 & 59 & 269 & 0,541052068 & 1,553343034 & 0,514959632 & 0,008988654 & 0,857167301 & foliation \\
\hline 50 & 47 & 271 & 0,750491578 & 1,588249619 & 0,681894488 & $-0,011902513$ & 0,731353702 & foliation \\
\hline 50 & 41 & 263 & 0,855211333 & 1,448623279 & 0,749084089 & 0,091975961 & 0,656059029 & foliation \\
\hline 50 & 43 & 262 & 0,820304748 & 1,431169987 & 0,724236218 & 0,101784763 & 0,68199836 & foliation \\
\hline 50 & 40 & 256 & 0,872664626 & 1,326450232 & 0,743289649 & 0,185322924 & 0,64278761 & foliation \\
\hline 50 & 35 & 275 & 0,959931089 & 1,658062789 & 0,816034923 & $-0,071393805$ & 0,573576436 & foliation \\
\hline 50 & 75 & 262 & 0,261799388 & 1,431169987 & 0,256300236 & 0,036020649 & 0,965925826 & foliation \\
\hline 50 & 65 & 285 & 0,436332313 & 1,832595715 & 0,408217894 & $-0,109381655$ & 0,906307787 & foliation \\
\hline 50 & 58 & 232 & 0,558505361 & 0,907571211 & 0,417582079 & 0,326250876 & 0,848048096 & foliation \\
\hline 50 & 38 & 262 & 0,907571211 & 1,431169987 & 0,780341887 & 0,1096699 & 0,615661475 & foliation \\
\hline 50 & 38 & 262 & 0,907571211 & 1,431169987 & 0,780341887 & 0,1096699 & 0,615661475 & foliation \\
\hline 50 & 43 & 257 & 0,820304748 & 1,343903524 & 0,712609154 & 0,164518786 & 0,68199836 & foliation \\
\hline 50 & 75 & 262 & 0,261799388 & 1,431169987 & 0,256300236 & 0,036020649 & 0,965925826 & foliation \\
\hline 50 & 65 & 285 & 0,436332313 & 1,832595715 & 0,408217894 & $-0,109381655$ & 0,906307787 & foliation \\
\hline 50 & 36 & 265 & 0,942477796 & 1,483529864 & 0,80593844 & 0,070510477 & 0,587785252 & foliation \\
\hline 50 & 45 & 260 & 0,785398163 & 1,396263402 & 0,69636424 & 0,122787804 & 0,707106781 & foliation \\
\hline 50 & 58 & 232 & 0,558505361 & 0,907571211 & 0,417582079 & 0,326250876 & 0,848048096 & foliation \\
\hline
\end{tabular}




\begin{tabular}{|c|c|c|c|c|c|c|c|c|}
\hline \multirow{2}{*}{ depth (meter) } & \multicolumn{2}{|c|}{ measures } & \multirow{2}{*}{$\theta$} & \multirow{2}{*}{$\phi$} & \multicolumn{3}{|c|}{ cosine director } & \multirow{2}{*}{$\begin{array}{l}\text { geological } \\
\text { structure }\end{array}$} \\
\hline & $\operatorname{dip}$ & dipdirection & & & $\mathbf{x}$ & $\mathbf{y}$ & $\mathbf{z}$ & \\
\hline 50 & 38 & 262 & 0,907571211 & 1,431169987 & 0,780341887 & 0,1096699 & 0,615661475 & foliation \\
\hline 50 & 43 & 257 & 0,820304748 & 1,343903524 & 0,712609154 & 0,164518786 & 0,68199836 & foliation \\
\hline 50 & 38 & 262 & 0,907571211 & 1,431169987 & 0,780341887 & 0,1096699 & 0,615661475 & foliation \\
\hline 50 & 40 & 260 & 0,872664626 & 1,396263402 & 0,754406507 & 0,133022222 & 0,64278761 & foliation \\
\hline 50 & 45 & 285 & 0,785398163 & 1,832595715 & 0,683012702 & $-0,183012702$ & 0,707106781 & foliation \\
\hline 50 & 16 & 240 & 1,291543646 & 1,047197551 & 0,832477048 & 0,480630848 & 0,275637356 & foliation \\
\hline 50 & 50 & 260 & 0,698131701 & 1,396263402 & 0,633022222 & 0,111618897 & 0,766044443 & foliation \\
\hline 50 & 45 & 275 & 0,785398163 & 1,658062789 & 0,704416026 & $-0,061628417$ & 0,707106781 & foliation \\
\hline 50 & 72 & 172 & 0,314159265 & $-0,13962634$ & $-0,043006853$ & 0,306009662 & 0,951056516 & joint \\
\hline 50 & 77 & 22 & 0,226892803 & $-2,757620218$ & $-0,084268148$ & \begin{tabular}{|l|}
$-0,208570986$ \\
\end{tabular} & 0,974370065 & joint \\
\hline 50 & 82 & 196 & 0,13962634 & 0,27925268 & 0,038361306 & 0,133781771 & 0,990268069 & joint \\
\hline 50 & 86 & 208 & 0,06981317 & 0,488692191 & 0,032748681 & 0,061591311 & 0,99756405 & joint \\
\hline 50 & 84 & 209 & 0,104719755 & 0,506145483 & 0,050676405 & 0,091422654 & 0,994521895 & joint \\
\hline 50 & 80 & 221 & 0,174532925 & 0,715584993 & 0,113923455 & 0,131053943 & 0,984807753 & joint \\
\hline 50 & 78 & 174 & 0,20943951 & $-0,104719755$ & $-0,02173269$ & 0,206772729 & 0,978147601 & joint \\
\hline 50 & 66 & 209 & 0,41887902 & 0,506145483 & 0,197189837 & 0,355739884 & 0,913545458 & joint \\
\hline 50 & 66 & 78 & 0,41887902 & $-1,780235837$ & $-0,397848472$ & $-0,084565303$ & 0,913545458 & joint \\
\hline 50 & 87 & 12 & 0,052359878 & $-2,932153143$ & $-0,010881257$ & $-0,05119229$ & 0,998629535 & joint \\
\hline 50 & 72 & 208 & 0,314159265 & 0,488692191 & 0,145074691 & 0,272845811 & 0,951056516 & joint \\
\hline 50 & 80 & 333 & 0,174532925 & 2,670353756 & 0,078834623 & $-0,154721659$ & 0,984807753 & joint \\
\hline 50 & 67 & 193 & 0,401425728 & 0,226892803 & 0,087895379 & 0,380716715 & 0,920504853 & joint \\
\hline 50 & 72 & 18 & 0,314159265 & $-2,827433388$ & $-0,095491503$ & \begin{tabular}{|l|}
$-0,293892626$ \\
\end{tabular} & 0,951056516 & joint \\
\hline 50 & 28 & 260 & 1,082104136 & 1,396263402 & 0,869533635 & 0,15332224 & 0,469471563 & joint \\
\hline 50 & 87 & 130 & 0,052359878 & $-0,872664626$ & $-0,040091668$ & 0,033640904 & 0,998629535 & joint \\
\hline 50 & 58 & 75 & 0,558505361 & $-1,832595715$ & $-0,511862703$ & $-0,137153198$ & 0,848048096 & joint \\
\hline 50 & 71 & 205 & 0,331612558 & 0,436332313 & 0,137591048 & 0,295064954 & 0,945518576 & joint \\
\hline 50 & 87 & 85 & 0,052359878 & $-1,658062789$ & $-0,052136802$ & $-0,004561379$ & 0,998629535 & joint \\
\hline 50 & 87 & 2 & 0,052359878 & $-3,106686069$ & \begin{tabular}{|l|}
$-0,001826499$ \\
\end{tabular} & $-0,052304075$ & 0,998629535 & joint \\
\hline 50 & 84 & 73 & 0,104719755 & $-1,8675023$ & $-0,099961067$ & $-0,030561165$ & 0,994521895 & joint \\
\hline 50 & 84 & 75 & 0,104719755 & $-1,832595715$ & $-0,100966742$ & $-0,027053957$ & 0,994521895 & joint \\
\hline 50 & 86 & 5 & 0,06981317 & $-3,054326191$ & $-0,006079677$ & $-0,069491029$ & 0,99756405 & joint \\
\hline 50 & 88 & 57 & 0,034906585 & $-2,14675498$ & $-0,029269181$ & $-0,019007628$ & 0,999390827 & joint \\
\hline 50 & 88 & 253 & 0,034906585 & 1,274090354 & 0,033374555 & 0,010203625 & 0,999390827 & joint \\
\hline 50 & 64 & 60 & 0,453785606 & $-2,094395102$ & $-0,379640549$ & $-0,219185573$ & 0,898794046 & joint \\
\hline 50 & 83 & 25 & 0,122173048 & $-2,705260341$ & $-0,05150421$ & $-0,110451135$ & 0,992546152 & joint \\
\hline 50 & 65 & 59 & 0,436332313 & $-2,111848395$ & $-0,362254555$ & $-0,217664496$ & 0,906307787 & joint \\
\hline 50 & 87 & 255 & 0,052359878 & 1,308996939 & 0,050552652 & 0,013545542 & 0,998629535 & joint \\
\hline 50 & 74 & 240 & 0,27925268 & 1,047197551 & 0,238708952 & 0,137818678 & 0,961261696 & joint \\
\hline 50 & 85 & 69 & 0,087266463 & $-1,93731547$ & $-0,081366895$ & $-0,031233825$ & 0,996194698 & joint \\
\hline 50 & 79 & 32 & 0,191986218 & $-2,583087293$ & $-0,101113362$ & $-0,161815205$ & 0,981627183 & joint \\
\hline 50 & 87 & 55 & 0,052359878 & $-2,181661565$ & $-0,042871106$ & $-0,030018671$ & 0,998629535 & joint \\
\hline 50 & 88 & 171 & 0,034906585 & $-0,157079633$ & $-0,005459484$ & 0,034469826 & 0,999390827 & joint \\
\hline 50 & 18 & 221 & 1,256637061 & 0,715584993 & 0,623949215 & 0,717771464 & 0,309016994 & joint \\
\hline 50 & 81 & 158 & 0,157079633 & $-0,383972435$ & $-0,058601382$ & 0,14504351 & 0,987688341 & joint \\
\hline 50 & 38 & 214 & 0,907571211 & 0,593411946 & 0,440650021 & 0,653290522 & 0,615661475 & joint \\
\hline 50 & 82 & 156 & 0,13962634 & $-0,41887902$ & $-0,0566068$ & 0,127140954 & 0,990268069 & joint \\
\hline 50 & 84 & 45 & 0,104719755 & $-2,35619449$ & $-0,073912785$ & $-0,073912785$ & 0,994521895 & joint \\
\hline 50 & 85 & 43 & 0,087266463 & $-2,391101075$ & $-0,059440074$ & $-0,063741675$ & 0,996194698 & joint \\
\hline 50 & 21 & 138 & 1,204277184 & $-0,733038286$ & $-0,624687237$ & 0,693785463 & 0,35836795 & joint \\
\hline 50 & 87 & 50 & 0,052359878 & $-2,268928028$ & $-0,040091668$ & $-0,033640904$ & 0,998629535 & joint \\
\hline 50 & 83 & 52 & 0,122173048 & $-2,234021443$ & $-0,096034353$ & $-0,07503026$ & 0,992546152 & joint \\
\hline 50 & 87 & 48 & 0,052359878 & $-2,303834613$ & $-0,038893195$ & $-0,03501959$ & 0,998629535 & joint \\
\hline 50 & 39 & 146 & 0,890117919 & $-0,593411946$ & $-0,434574507$ & 0,644283201 & 0,629320391 & joint \\
\hline 50 & 88 & 51 & 0,034906585 & $-2,251474735$ & $-0,027122003$ & $-0,021962965$ & 0,999390827 & joint \\
\hline 50 & 87 & 265 & 0,052359878 & 1,483529864 & 0,052136802 & 0,004561379 & 0,998629535 & joint \\
\hline 50 & 87 & 68 & 0,052359878 & $-1,954768762$ & $-0,048525054$ & $-0,019605394$ & 0,998629535 & joint \\
\hline 50 & 83 & 261 & 0,122173048 & 1,413716694 & 0,12036893 & 0,019064566 & 0,992546152 & joint \\
\hline
\end{tabular}




\begin{tabular}{|c|c|c|c|c|c|c|c|c|}
\hline \multirow{2}{*}{ depth (meter) } & \multicolumn{2}{|c|}{ measures } & \multirow{2}{*}{$\theta$} & \multirow{2}{*}{$\phi$} & \multicolumn{3}{|c|}{ cosine director } & \multirow{2}{*}{$\begin{array}{l}\text { geological } \\
\text { structure }\end{array}$} \\
\hline & $\operatorname{dip}$ & dipdirection & & & $\mathbf{x}$ & $\mathbf{y}$ & $\mathbf{z}$ & \\
\hline 50 & 83 & 271 & 0,122173048 & 1,588249619 & 0,121850782 & $-0,002126913$ & 0,992546152 & joint \\
\hline 50 & 83 & 261 & 0,122173048 & 1,413716694 & 0,12036893 & 0,019064566 & 0,992546152 & joint \\
\hline 50 & 87 & 64 & 0,052359878 & $-2,024581932$ & $-0,047039246$ & $-0,022942573$ & 0,998629535 & joint \\
\hline 50 & 82 & 256 & 0,13962634 & 1,326450232 & 0,135039065 & \begin{tabular}{|l}
0,03366902 \\
\end{tabular} & 0,990268069 & joint \\
\hline 50 & 87 & 172 & 0,052359878 & $-0,13962634$ & $-0,007283757$ & 0,051826626 & 0,998629535 & joint \\
\hline 50 & 87 & 248 & 0,052359878 & 1,186823891 & 0,048525054 & 0,019605394 & 0,998629535 & joint \\
\hline 50 & 85 & 170 & 0,087266463 & $-0,174532925$ & $-0,015134436$ & 0,085831651 & 0,996194698 & joint \\
\hline 50 & 70 & 124 & 0,34906585 & $-0,977384381$ & $-0,283547549$ & 0,191255237 & 0,939692621 & joint \\
\hline 50 & 38 & 256 & 0,907571211 & 1,326450232 & 0,764603466 & 0,190637055 & 0,615661475 & joint \\
\hline 50 & 76 & 226 & 0,244346095 & 0,802851456 & 0,174024048 & 0,16805307 & 0,970295726 & joint \\
\hline 50 & 74 & 75 & 0,27925268 & $-1,832595715$ & $-0,266245241$ & $-0,071340197$ & 0,961261696 & joint \\
\hline 50 & 70 & 242 & 0,34906585 & 1,082104136 & 0,301985862 & 0,160568731 & 0,939692621 & joint \\
\hline 50 & 78 & 238 & 0,20943951 & 1,012290966 & 0,176319114 & 0,11017641 & 0,978147601 & joint \\
\hline 50 & 75 & 235 & 0,261799388 & 0,959931089 & 0,21201215 & 0,148452506 & 0,965925826 & joint \\
\hline 50 & 80 & 240 & 0,174532925 & 1,047197551 & 0,150383733 & 0,086824089 & 0,984807753 & joint \\
\hline 50 & 70 & 234 & 0,34906585 & 0,942477796 & 0,276700108 & 0,201034396 & 0,939692621 & joint \\
\hline 50 & 83 & 237 & 0,122173048 & 0,994837674 & 0,102208231 & 0,066374802 & 0,992546152 & joint \\
\hline 50 & 80 & 280 & 0,174532925 & 1,745329252 & 0,171010072 & $-0,03015369$ & 0,984807753 & joint \\
\hline 50 & 36 & 365 & 0,942477796 & 3,228859116 & $-0,070510477$ & $-0,80593844$ & 0,587785252 & joint \\
\hline 50 & 85 & 78 & 0,087266463 & $-1,780235837$ & $-0,085251181$ & $-0,018120698$ & 0,996194698 & joint \\
\hline 50 & 61 & 43 & 0,506145483 & $-2,391101075$ & $-0,330639366$ & $-0,35456731$ & 0,874619707 & joint \\
\hline 50 & 60 & 145 & 0,523598776 & $-0,610865238$ & $-0,286788218$ & 0,409576022 & 0,866025404 & joint \\
\hline 50 & 81 & 355 & 0,157079633 & 3,054326191 & 0,013634162 & $-0,155839185$ & 0,987688341 & joint \\
\hline 50 & 76 & 83 & 0,244346095 & $-1,692969374$ & $-0,240118646$ & $-0,029482863$ & 0,970295726 & joint \\
\hline 50 & 78 & 5 & 0,20943951 & $-3,054326191$ & $-0,018120698$ & $-0,207120524$ & 0,978147601 & joint \\
\hline 50 & 66 & 50 & 0,41887902 & $-2,268928028$ & $-0,311578345$ & $-0,261445275$ & 0,913545458 & joint \\
\hline 50 & 75 & 38 & 0,261799388 & $-2,478367538$ & $-0,159344915$ & $-0,203952191$ & 0,965925826 & joint \\
\hline 50 & 88 & 345 & 0,034906585 & 2,879793266 & 0,009032654 & $-0,033710325$ & 0,999390827 & joint \\
\hline 50 & 85 & 76 & 0,087266463 & $-1,815142422$ & $-0,084566845$ & $-0,021084882$ & 0,996194698 & joint \\
\hline 50 & 60 & 145 & 0,523598776 & $-0,610865238$ & $-0,286788218$ & 0,409576022 & 0,866025404 & joint \\
\hline 50 & 74 & 219 & 0,27925268 & 0,680678408 & 0,173464209 & 0,214210458 & 0,961261696 & joint \\
\hline 50 & 61 & 43 & 0,506145483 & $-2,391101075$ & $-0,330639366$ & $-0,35456731$ & 0,874619707 & joint \\
\hline 50 & 87 & 32 & 0,052359878 & $-2,583087293$ & $-0,027733831$ & $-0,044383408$ & 0,998629535 & joint \\
\hline 50 & 45 & 253 & 0,785398163 & 1,274090354 & 0,676209578 & 0,206738015 & 0,707106781 & joint \\
\hline 50 & 75 & 58 & 0,261799388 & $-2,129301687$ & $-0,219490998$ & $-0,137153198$ & 0,965925826 & joint \\
\hline 50 & 77 & 51 & 0,226892803 & $-2,251474735$ & $-0,174819803$ & $-0,141566285$ & 0,974370065 & joint \\
\hline 50 & 62 & 55 & 0,488692191 & $-2,181661565$ & $-0,38456859$ & $-0,269277826$ & 0,882947593 & joint \\
\hline 50 & 31 & 83 & 1,029744259 & $-1,692969374$ & $-0,850778106$ & $-0,104462416$ & 0,515038075 & joint \\
\hline 50 & 53 & 125 & 0,645771823 & $-0,959931089$ & $-0,492978006$ & 0,345186916 & 0,79863551 & joint \\
\hline 50 & 77 & 60 & 0,226892803 & $-2,094395102$ & $-0,194813328$ & $-0,112475527$ & 0,974370065 & joint \\
\hline 50 & 81 & 41 & 0,157079633 & $-2,42600766$ & $-0,102630243$ & $-0,118062589$ & 0,987688341 & joint \\
\hline 50 & 77 & 83 & 0,226892803 & $-1,692969374$ & $-0,223274303$ & $-0,027414637$ & 0,974370065 & joint \\
\hline 50 & 56 & 289 & 0,593411946 & 1,902408885 & 0,528727278 & $-0,182055402$ & 0,829037573 & foliation \\
\hline 50 & 41 & 262 & 0,855211333 & 1,431169987 & 0,747364798 & 0,105035273 & 0,656059029 & foliation \\
\hline 50 & 47 & 266 & 0,750491578 & 1,500983157 & 0,680337046 & 0,047573801 & 0,731353702 & foliation \\
\hline 50 & 45 & 270 & 0,785398163 & 1,570796327 & 0,707106781 & $4,33155 \mathrm{E}-17$ & 0,707106781 & foliation \\
\hline 50 & 54 & 285 & 0,628318531 & 1,832595715 & 0,567756956 & $-0,152130018$ & 0,809016994 & foliation \\
\hline 50 & 44 & 282 & 0,802851456 & 1,780235837 & 0,7036205 & $-0,149559154$ & 0,69465837 & foliation \\
\hline 50 & 54 & 262 & 0,628318531 & 1,431169987 & 0,582064967 & 0,081803896 & 0,809016994 & foliation \\
\hline 50 & 41 & 286 & 0,855211333 & 1,850049007 & 0,725473411 & $-0,208026153$ & 0,656059029 & foliation \\
\hline 50 & 46 & 291 & 0,767944871 & 1,93731547 & 0,648519458 & $-0,248943296$ & 0,7193398 & foliation \\
\hline 50 & 46 & 252 & 0,767944871 & 1,256637061 & 0,66065937 & 0,214661242 & 0,7193398 & foliation \\
\hline 50 & 47 & 258 & 0,750491578 & 1,361356817 & 0,66709506 & 0,141795432 & 0,731353702 & foliation \\
\hline 50 & 64 & 273 & 0,453785606 & 1,623156204 & 0,437770374 & $-0,022942573$ & 0,898794046 & foliation \\
\hline 50 & 54 & 272 & 0,628318531 & 1,605702912 & 0,587427189 & $-0,020513409$ & 0,809016994 & foliation \\
\hline 50 & 58 & 264 & 0,558505361 & 1,466076572 & 0,527016311 & 0,055391646 & 0,848048096 & foliation \\
\hline 50 & 63 & 294 & 0,471238898 & 1,989675347 & 0,414740959 & $-0,184654572$ & 0,891006524 & foliation \\
\hline
\end{tabular}




\begin{tabular}{|c|c|c|c|c|c|c|c|c|}
\hline \multirow{2}{*}{ depth (meter) } & \multicolumn{2}{|c|}{ measures } & \multirow{2}{*}{$\boldsymbol{\theta}$} & \multirow{2}{*}{$\phi$} & \multicolumn{3}{|c|}{ cosine director } & \multirow{2}{*}{$\begin{array}{l}\text { geological } \\
\text { structure }\end{array}$} \\
\hline & dip & dipdirection & & & $\mathbf{x}$ & $\mathbf{y}$ & $\mathbf{z}$ & \\
\hline 50 & 58 & 285 & 0,558505361 & 1,832595715 & 0,511862703 & $-0,137153198$ & 0,848048096 & foliation \\
\hline 50 & 53 & 274 & 0,645771823 & 1,640609497 & 0,600349032 & $-0,041980494$ & 0,79863551 & foliation \\
\hline 50 & 57 & 287 & 0,575958653 & 1,8675023 & 0,520840899 & $-0,159237043$ & 0,838670568 & foliation \\
\hline 50 & 58 & 288 & 0,558505361 & 1,884955592 & 0,503983169 & $-0,163754058$ & 0,848048096 & foliation \\
\hline 50 & 58 & 295 & 0,558505361 & 2,00712864 & 0,480269956 & $-0,223953558$ & 0,848048096 & foliation \\
\hline 50 & 57 & 291 & 0,575958653 & 1,93731547 & 0,508464343 & $-0,195181174$ & 0,838670568 & foliation \\
\hline 50 & 54 & 292 & 0,628318531 & 1,954768762 & 0,544984996 & $-0,220188231$ & 0,809016994 & foliation \\
\hline 50 & 56 & 292 & 0,593411946 & 1,954768762 & 0,518474632 & $-0,209477349$ & 0,829037573 & foliation \\
\hline 50 & 57 & 289 & 0,575958653 & 1,902408885 & 0,514966325 & $-0,177317125$ & 0,838670568 & foliation \\
\hline 50 & 50 & 278 & 0,698131701 & 1,710422667 & 0,636532045 & $-0,089458745$ & 0,766044443 & foliation \\
\hline 50 & 52 & 275 & 0,663225116 & 1,658062789 & 0,613318698 & $-0,053658433$ & 0,788010754 & foliation \\
\hline 50 & 40 & 288 & 0,872664626 & 1,884955592 & 0,728551559 & $-0,236720751$ & 0,64278761 & foliation \\
\hline 50 & 57 & 268 & 0,575958653 & 1,535889742 & 0,544307256 & 0,019007628 & 0,838670568 & foliation \\
\hline 50 & 54 & 265 & 0,628318531 & 1,483529864 & 0,585548552 & 0,05122886 & 0,809016994 & foliation \\
\hline 50 & 53 & 75 & 0,645771823 & $-1,832595715$ & $-0,581308674$ & $-0,15576119$ & 0,79863551 & joint \\
\hline 50 & 85 & 62 & 0,087266463 & $-2,059488517$ & $-0,076953953$ & $-0,040917143$ & 0,996194698 & joint \\
\hline 50 & 87 & 210 & 0,052359878 & 0,523598776 & 0,026167978 & 0,045324268 & 0,998629535 & joint \\
\hline 50 & 78 & 200 & 0,20943951 & 0,34906585 & 0,071109986 & 0,195373082 & 0,978147601 & joint \\
\hline 50 & 81 & 343 & 0,157079633 & 2,844886681 & 0,045737011 & $-0,149599023$ & 0,987688341 & joint \\
\hline 50 & 75 & 224 & 0,261799388 & 0,767944871 & 0,179790816 & 0,18617884 & 0,965925826 & joint \\
\hline 50 & 88 & 55 & 0,034906585 & $-2,181661565$ & $-0,028587994$ & $-0,020017529$ & 0,999390827 & joint \\
\hline 50 & 63 & 56 & 0,471238898 & $-2,164208272$ & $-0,376375182$ & $-0,253868266$ & 0,891006524 & joint \\
\hline 50 & 68 & 59 & 0,383972435 & $-2,111848395$ & $-0,321100523$ & $-0,192936659$ & 0,927183855 & joint \\
\hline 50 & 33 & 270 & 0,994837674 & 1,570796327 & 0,838670568 & 5,13748E-17 & 0,544639035 & joint \\
\hline 50 & 61 & 54 & 0,506145483 & $-2,199114858$ & $-0,392219222$ & $-0,284963945$ & 0,874619707 & joint \\
\hline 50 & 63 & 58 & 0,471238898 & $-2,129301687$ & $-0,385005779$ & $-0,240578312$ & 0,891006524 & joint \\
\hline 50 & 71 & 33 & 0,331612558 & $-2,565634$ & $-0,177317125$ & $-0,273044429$ & 0,945518576 & joint \\
\hline 50 & 89 & 45 & 0,017453293 & $-2,35619449$ & $-0,012340715$ & $-0,012340715$ & 0,999847695 & joint \\
\hline 50 & 77 & 49 & 0,226892803 & $-2,28638132$ & $-0,169772716$ & $-0,14758117$ & 0,974370065 & joint \\
\hline 50 & 69 & 312 & 0,366519143 & 2,303834613 & 0,266319287 & $-0,239794963$ & 0,933580426 & joint \\
\hline 50 & 84 & 314 & 0,104719755 & 2,338741198 & 0,075191484 & $-0,072611572$ & 0,994521895 & joint \\
\hline 50 & 44 & 143 & 0,802851456 & $-0,645771823$ & $-0,432909499$ & 0,574490308 & 0,69465837 & joint \\
\hline 50 & 63 & 348 & 0,471238898 & 2,932153143 & 0,094389932 & $-0,444069718$ & 0,891006524 & joint \\
\hline 50 & 71 & 35 & 0,331612558 & $-2,530727415$ & $-0,186738222$ & $-0,266689819$ & 0,945518576 & joint \\
\hline 50 & 63 & 348 & 0,471238898 & 2,932153143 & 0,094389932 & $-0,444069718$ & 0,891006524 & joint \\
\hline 50 & 39 & 142 & 0,890117919 & $-0,663225116$ & $-0,478458829$ & 0,612399375 & 0,629320391 & joint \\
\hline 50 & 85 & 214 & 0,087266463 & 0,593411946 & 0,048736873 & 0,072255385 & 0,996194698 & joint \\
\hline 50 & 29 & 301 & 1,064650844 & 2,111848395 & 0,749695414 & $-0,45046245$ & 0,48480962 & joint \\
\hline 50 & 87 & 339 & 0,052359878 & 2,775073511 & 0,018755529 & $-0,048859824$ & 0,998629535 & joint \\
\hline 50 & 46 & 309 & 0,767944871 & 2,251474735 & 0,539850947 & $-0,437162677$ & 0,7193398 & joint \\
\hline 50 & 71 & 14 & 0,331612558 & $-2,897246558$ & $-0,078762065$ & $-0,315897389$ & 0,945518576 & joint \\
\hline 50 & 67 & 90 & 0,401425728 & $-1,570796327$ & $-0,390731128$ & $2,39352 \mathrm{E}-17$ & 0,920504853 & joint \\
\hline 50 & 69 & 172 & 0,366519143 & $-0,13962634$ & $-0,049875179$ & 0,354880337 & 0,933580426 & joint \\
\hline 50 & 64 & 355 & 0,453785606 & 3,054326191 & 0,038206563 & $-0,436703012$ & 0,898794046 & joint \\
\hline 50 & 57 & 73 & 0,575958653 & $-1,8675023$ & $-0,520840899$ & $-0,159237043$ & 0,838670568 & joint \\
\hline 50 & 87 & 196 & 0,052359878 & 0,27925268 & 0,014425745 & 0,05030855 & 0,998629535 & joint \\
\hline 50 & 81 & 330 & 0,157079633 & 2,617993878 & 0,078217233 & $-0,135476221$ & 0,987688341 & joint \\
\hline 50 & 85 & 83 & 0,087266463 & $-1,692969374$ & $-0,086506097$ & $-0,010621613$ & 0,996194698 & joint \\
\hline 50 & 62 & 65 & 0,488692191 & $-2,00712864$ & $-0,425485733$ & $-0,198407256$ & 0,882947593 & joint \\
\hline 50 & 76 & 83 & 0,244346095 & $-1,692969374$ & $-0,240118646$ & $-0,029482863$ & 0,970295726 & joint \\
\hline 50 & 66 & 50 & 0,41887902 & $-2,268928028$ & $-0,311578345$ & $-0,261445275$ & 0,913545458 & joint \\
\hline 50 & 35 & 100 & 0,959931089 & $-1,396263402$ & $-0,806707284$ & 0,14224426 & 0,573576436 & joint \\
\hline 50 & 49 & 262 & 0,715584993 & 1,431169987 & 0,649674308 & 0,091305769 & 0,75470958 & foliation \\
\hline 50 & 46 & 258 & 0,767944871 & 1,361356817 & 0,679478418 & 0,144427596 & 0,7193398 & foliation \\
\hline 50 & 46 & 281 & 0,767944871 & 1,762782545 & 0,68189554 & $-0,132547066$ & 0,7193398 & foliation \\
\hline 50 & 45 & 287 & 0,785398163 & 1,8675023 & 0,676209578 & $-0,206738015$ & 0,707106781 & foliation \\
\hline 50 & 41 & 287 & 0,855211333 & 1,8675023 & 0,721732361 & $-0,220655727$ & 0,656059029 & foliation \\
\hline
\end{tabular}




\begin{tabular}{|c|c|c|c|c|c|c|c|c|}
\hline \multirow{2}{*}{ depth (meter) } & \multicolumn{2}{|c|}{ measures } & \multirow{2}{*}{$\theta$} & \multirow{2}{*}{$\phi$} & \multicolumn{3}{|c|}{ cosine director } & \multirow{2}{*}{$\begin{array}{l}\text { geological } \\
\text { structure }\end{array}$} \\
\hline & $\operatorname{dip}$ & dipdirection & & & $\mathbf{x}$ & $\mathbf{y}$ & $\mathbf{z}$ & \\
\hline 50 & 43 & 295 & 0,820304748 & 2,00712864 & 0,662831555 & $-0,30908343$ & 0,68199836 & foliation \\
\hline 50 & 39 & 300 & 0,890117919 & 2,094395102 & 0,673028145 & $-0,388572981$ & 0,629320391 & foliation \\
\hline 50 & 40 & 296 & 0,872664626 & 2,024581932 & 0,688516185 & $-0,335811781$ & 0,64278761 & foliation \\
\hline 50 & 89 & 194 & 0,017453293 & 0,244346095 & 0,004222119 & 0,016933995 & 0,999847695 & foliation \\
\hline 50 & 41 & 285 & 0,855211333 & 1,832595715 & 0,728993475 & $-0,195333213$ & 0,656059029 & foliation \\
\hline 50 & 43 & 280 & 0,820304748 & 1,745329252 & 0,720242796 & $-0,126998238$ & 0,68199836 & foliation \\
\hline 50 & 50 & 261 & 0,698131701 & 1,413716694 & 0,634873828 & 0,100554136 & 0,766044443 & foliation \\
\hline 50 & 51 & 286 & 0,680678408 & 1,850049007 & 0,604941586 & $-0,173464209$ & 0,777145961 & foliation \\
\hline 50 & 52 & 280 & 0,663225116 & 1,745329252 & 0,606308194 & $-0,106908493$ & 0,788010754 & foliation \\
\hline 50 & 40 & 246 & 0,872664626 & 1,151917306 & 0,699816421 & 0,311578345 & 0,64278761 & foliation \\
\hline 50 & 51 & 276 & 0,680678408 & 1,675516082 & 0,625872908 & $-0,065781893$ & 0,777145961 & foliation \\
\hline 50 & 53 & 274 & 0,645771823 & 1,640609497 & 0,600349032 & $-0,041980494$ & 0,79863551 & foliation \\
\hline 50 & 49 & 281 & 0,715584993 & 1,762782545 & 0,644005377 & $-0,125181964$ & 0,75470958 & foliation \\
\hline 50 & 41 & 285 & 0,855211333 & 1,832595715 & 0,728993475 & $-0,195333213$ & 0,656059029 & foliation \\
\hline 50 & 35 & 252 & 0,959931089 & 1,256637061 & 0,77905989 & 0,253131903 & 0,573576436 & foliation \\
\hline 50 & 58 & 267 & 0,558505361 & 1,518436449 & 0,529193028 & 0,027733831 & 0,848048096 & foliation \\
\hline 50 & 57 & 246 & 0,575958653 & 1,151917306 & 0,497552516 & 0,221524653 & 0,838670568 & foliation \\
\hline 50 & 40 & 291 & 0,872664626 & 1,93731547 & 0,715164098 & $-0,274525776$ & 0,64278761 & foliation \\
\hline 50 & 49 & 273 & 0,715584993 & 1,623156204 & 0,655159923 & $-0,034335477$ & 0,75470958 & foliation \\
\hline 50 & 48 & 275 & 0,733038286 & 1,658062789 & 0,666584362 & $-0,058318575$ & 0,743144825 & foliation \\
\hline 50 & 55 & 289 & 0,610865238 & 1,902408885 & 0,542327175 & $-0,186738222$ & 0,819152044 & foliation \\
\hline 50 & 49 & 273 & 0,715584993 & 1,623156204 & 0,655159923 & $-0,034335477$ & 0,75470958 & foliation \\
\hline 50 & 48 & 274 & 0,733038286 & 1,640609497 & 0,667500638 & $-0,046676192$ & 0,743144825 & foliation \\
\hline 50 & 49 & 284 & 0,715584993 & 1,815142422 & 0,636571272 & $-0,158715044$ & 0,75470958 & foliation \\
\hline 50 & 51 & 277 & 0,680678408 & 1,692969374 & 0,624629532 & $-0,076694863$ & 0,777145961 & foliation \\
\hline 50 & 48 & 276 & 0,733038286 & 1,675516082 & 0,665465039 & $-0,069943194$ & 0,743144825 & foliation \\
\hline 50 & 46 & 272 & 0,767944871 & 1,605702912 & 0,694235203 & $-0,024243228$ & 0,7193398 & foliation \\
\hline 50 & 43 & 268 & 0,820304748 & 1,535889742 & 0,730908181 & 0,025523876 & 0,68199836 & foliation \\
\hline 50 & 49 & 278 & 0,715584993 & 1,710422667 & 0,649674308 & $-0,091305769$ & 0,75470958 & foliation \\
\hline 50 & 51 & 262 & 0,680678408 & 1,431169987 & 0,623195888 & 0,08758447 & 0,777145961 & foliation \\
\hline 50 & 45 & 282 & 0,785398163 & 1,780235837 & 0,691654801 & $-0,147015766$ & 0,707106781 & foliation \\
\hline 50 & 45 & 275 & 0,785398163 & 1,658062789 & 0,704416026 & $-0,061628417$ & 0,707106781 & foliation \\
\hline 50 & 45 & 260 & 0,785398163 & 1,396263402 & 0,69636424 & 0,122787804 & 0,707106781 & foliation \\
\hline 50 & 41 & 286 & 0,855211333 & 1,850049007 & 0,725473411 & $-0,208026153$ & 0,656059029 & joint \\
\hline 50 & 28 & 260 & 1,082104136 & 1,396263402 & 0,869533635 & 0,15332224 & 0,469471563 & joint \\
\hline 50 & 60 & 228 & 0,523598776 & 0,837758041 & 0,371572413 & 0,334565303 & 0,866025404 & joint \\
\hline 50 & 61 & 195 & 0,506145483 & 0,261799388 & 0,125477963 & 0,468290133 & 0,874619707 & joint \\
\hline 50 & 88 & 75 & 0,034906585 & $-1,832595715$ & $-0,033710325$ & $-0,009032654$ & 0,999390827 & joint \\
\hline 50 & 86 & 47 & 0,06981317 & $-2,321287905$ & $-0,051016655$ & $-0,047573801$ & 0,99756405 & joint \\
\hline 50 & 46 & 282 & 0,767944871 & 1,780235837 & 0,679478418 & $-0,144427596$ & 0,7193398 & joint \\
\hline 50 & 52 & 41 & 0,663225116 & $-2,42600766$ & $-0,40391027$ & $-0,464645614$ & 0,788010754 & joint \\
\hline 50 & 53 & 72 & 0,645771823 & $-1,884955592$ & $-0,572360099$ & $-0,18597107$ & 0,79863551 & joint \\
\hline 50 & 87 & 250 & 0,052359878 & 1,221730476 & 0,049179712 & 0,017899951 & 0,998629535 & joint \\
\hline 50 & 52 & 198 & 0,663225116 & 0,314159265 & 0,190249859 & 0,585528858 & 0,788010754 & joint \\
\hline 50 & 63 & 57 & 0,471238898 & $-2,14675498$ & $-0,38074847$ & $-0,247260948$ & 0,891006524 & joint \\
\hline 50 & 83 & 217 & 0,122173048 & 0,645771823 & 0,073342802 & 0,097329185 & 0,992546152 & joint \\
\hline 50 & 87 & 47 & 0,052359878 & $-2,321287905$ & $-0,038276095$ & $-0,035693036$ & 0,998629535 & joint \\
\hline 50 & 87 & 54 & 0,052359878 & $-2,199114858$ & $-0,042340678$ & $-0,030762303$ & 0,998629535 & joint \\
\hline 50 & 55 & 127 & 0,610865238 & $-0,925024504$ & $-0,45807851$ & 0,345186916 & 0,819152044 & joint \\
\hline 50 & 44 & 80 & 0,802851456 & $-1,745329252$ & $-0,708411412$ & $-0,124912045$ & 0,69465837 & joint \\
\hline 50 & 70 & 169 & 0,34906585 & $-0,191986218$ & $-0,06526052$ & 0,33573627 & 0,939692621 & joint \\
\hline 50 & 85 & 300 & 0,087266463 & 2,094395102 & 0,075479087 & $-0,043577871$ & 0,996194698 & joint \\
\hline 50 & 59 & 264 & 0,541052068 & 1,466076572 & 0,512216642 & 0,053836138 & 0,857167301 & joint \\
\hline 50 & 65 & 319 & 0,436332313 & 2,42600766 & 0,277262526 & $-0,318954051$ & 0,906307787 & joint \\
\hline 50 & 87 & 66 & 0,052359878 & $-1,989675347$ & $-0,047811275$ & $-0,021286951$ & 0,998629535 & joint \\
\hline 50 & 22 & 286 & 1,186823891 & 1,850049007 & 0,891266324 & $-0,255566506$ & 0,374606593 & joint \\
\hline 50 & 52 & 193 & 0,663225116 & 0,226892803 & 0,138493698 & 0,599882112 & 0,788010754 & joint \\
\hline
\end{tabular}




\begin{tabular}{|c|c|c|c|c|c|c|c|c|}
\hline \multirow{2}{*}{ depth (meter) } & \multicolumn{2}{|c|}{ measures } & \multirow{2}{*}{$\theta$} & \multirow{2}{*}{$\phi$} & \multicolumn{3}{|c|}{ cosine director } & \multirow{2}{*}{$\begin{array}{l}\text { geological } \\
\text { structure }\end{array}$} \\
\hline & $\operatorname{dip}$ & dipdirection & & & $\mathbf{x}$ & $\mathbf{y}$ & $\mathbf{z}$ & \\
\hline 50 & 51 & 204 & 0,680678408 & 0,41887902 & 0,255967663 & 0,574912785 & 0,777145961 & joint \\
\hline 50 & 89 & 230 & 0,017453293 & 0,872664626 & 0,013369319 & 0,011218191 & 0,999847695 & joint \\
\hline 50 & 85 & 210 & 0,087266463 & 0,523598776 & 0,043577871 & 0,075479087 & 0,996194698 & joint \\
\hline 50 & 87 & 200 & 0,052359878 & 0,34906585 & 0,017899951 & 0,049179712 & 0,998629535 & joint \\
\hline 50 & 84 & 268 & 0,104719755 & 1,535889742 & 0,104464787 & 0,003647991 & 0,994521895 & joint \\
\hline 50 & 57 & 338 & 0,575958653 & 2,757620218 & 0,204025374 & $-0,50498052$ & 0,838670568 & joint \\
\hline 50 & 78 & 45 & 0,20943951 & $-2,35619449$ & $-0,147015766$ & \begin{tabular}{|l|}
$-0,147015766$ \\
\end{tabular} & 0,978147601 & joint \\
\hline 50 & 88 & 345 & 0,034906585 & 2,879793266 & 0,009032654 & $-0,033710325$ & 0,999390827 & joint \\
\hline 50 & 37 & 265 & 0,925024504 & 1,483529864 & 0,795596461 & 0,069605671 & 0,601815023 & foliation \\
\hline 50 & 87 & 53 & 0,052359878 & $-2,21656815$ & $-0,041797353$ & $-0,031496565$ & 0,998629535 & joint \\
\hline 50 & 68 & 269 & 0,383972435 & 1,553343034 & 0,374549539 & 0,006537787 & 0,927183855 & foliation \\
\hline 50 & 89 & 85 & 0,017453293 & $-1,658062789$ & $-0,017385995$ & $-0,001521077$ & 0,999847695 & foliation \\
\hline 50 & 72 & 36 & 0,314159265 & $-2,513274123$ & $-0,181635632$ & $-0,25$ & 0,951056516 & joint \\
\hline 50 & 84 & 209 & 0,104719755 & 0,506145483 & 0,050676405 & 0,091422654 & 0,994521895 & joint \\
\hline 50 & 89 & 225 & 0,017453293 & 0,785398163 & 0,012340715 & 0,012340715 & 0,999847695 & joint \\
\hline 50 & 47 & 274 & 0,750491578 & 1,640609497 & 0,680337046 & $-0,047573801$ & 0,731353702 & foliation \\
\hline 50 & 59 & 273 & 0,541052068 & 1,623156204 & 0,514332233 & $-0,02695501$ & 0,857167301 & foliation \\
\hline 50 & 57 & 293 & 0,575958653 & 1,972222055 & 0,501342875 & $-0,212807425$ & 0,838670568 & foliation \\
\hline 50 & 77 & 135 & 0,226892803 & $-0,785398163$ & $-0,159064416$ & 0,159064416 & 0,974370065 & joint \\
\hline 50 & 67 & 328 & 0,401425728 & 2,583087293 & 0,207055952 & $-0,33135879$ & 0,920504853 & joint \\
\hline 50 & 71 & 25 & 0,331612558 & $-2,705260341$ & $-0,137591048$ & $-0,295064954$ & 0,945518576 & joint \\
\hline 50 & 40 & 256 & 0,872664626 & 1,326450232 & 0,743289649 & 0,185322924 & 0,64278761 & joint \\
\hline 50 & 77 & 45 & 0,226892803 & $-2,35619449$ & $-0,159064416$ & $-0,159064416$ & 0,974370065 & joint \\
\hline 50 & 48 & 320 & 0,733038286 & 2,443460953 & 0,430108863 & $-0,512583783$ & 0,743144825 & joint \\
\hline 50 & 32 & 119 & 1,012290966 & $-1,064650844$ & $-0,741719578$ & 0,411141875 & 0,529919264 & joint \\
\hline 50 & 32 & 312 & 1,012290966 & 2,303834613 & 0,630222554 & $-0,567454937$ & 0,529919264 & joint \\
\hline 50 & 81 & 230 & 0,157079633 & 0,872664626 & 0,119835753 & 0,100554136 & 0,987688341 & joint \\
\hline 50 & 89 & 220 & 0,017453293 & 0,698131701 & 0,011218191 & 0,013369319 & 0,999847695 & joint \\
\hline 50 & 40 & 260 & 0,872664626 & 1,396263402 & 0,754406507 & 0,133022222 & 0,64278761 & joint \\
\hline 50 & 22 & 251 & 1,186823891 & 1,239183769 & 0,8766695557 & 0,301861536 & 0,374606593 & joint \\
\hline 50 & 61 & 280 & 0,506145483 & 1,745329252 & 0,477444273 & $-0,084186307$ & 0,874619707 & joint \\
\hline 50 & 58 & 285 & 0,558505361 & 1,832595715 & 0,511862703 & $-0,137153198$ & 0,848048096 & joint \\
\hline 50 & 85 & 246 & 0,087266463 & 1,151917306 & 0,079620733 & 0,035449434 & 0,996194698 & joint \\
\hline 50 & 63 & 286 & 0,471238898 & 1,850049007 & 0,436403678 & $-0,125136741$ & 0,891006524 & foliation \\
\hline 50 & 49 & 286 & 0,715584993 & 1,850049007 & 0,630644415 & $-0,180834376$ & 0,75470958 & foliation \\
\hline 50 & 57 & 268 & 0,575958653 & 1,535889742 & 0,544307256 & 0,019007628 & 0,838670568 & foliation \\
\hline 50 & 51 & 259 & 0,680678408 & 1,378810109 & 0,617758003 & 0,120079992 & 0,777145961 & foliation \\
\hline 50 & 55 & 279 & 0,610865238 & 1,727875959 & 0,566514759 & $-0,089727123$ & 0,819152044 & foliation \\
\hline 50 & 43 & 283 & 0,820304748 & 1,79768913 & 0,712609154 & $-0,164518786$ & 0,68199836 & foliation \\
\hline 50 & 48 & 260 & 0,733038286 & 1,396263402 & 0,658965009 & 0,11619331 & 0,743144825 & foliation \\
\hline 50 & 41 & 280 & 0,855211333 & 1,745329252 & 0,743243846 & $-0,131053943$ & 0,656059029 & foliation \\
\hline 50 & 26 & 116 & 1,117010721 & $-1,117010721$ & $-0,807830738$ & 0,394005377 & 0,438371147 & joint \\
\hline 50 & 47 & 278 & 0,750491578 & 1,710422667 & 0,675361199 & $-0,094915827$ & 0,731353702 & foliation \\
\hline 50 & 43 & 278 & 0,820304748 & 1,710422667 & 0,724236218 & $-0,101784763$ & 0,68199836 & foliation \\
\hline 50 & 55 & 26 & 0,610865238 & $-2,687807048$ & $-0,25143936$ & $-0,515527086$ & 0,819152044 & joint \\
\hline 50 & 89 & 331 & 0,017453293 & 2,635447171 & 0,008461095 & $-0,015264219$ & 0,999847695 & joint \\
\hline 50 & 89 & 240 & 0,017453293 & 1,047197551 & 0,015114227 & 0,008726203 & 0,999847695 & joint \\
\hline 50 & 24 & 244 & 1,151917306 & 1,117010721 & 0,821089218 & 0,40047197 & 0,406736643 & joint \\
\hline 50 & 33 & 242 & 0,994837674 & 1,082104136 & 0,740502159 & 0,393731982 & 0,544639035 & joint \\
\hline 50 & 27 & 135 & 1,099557429 & $-0,785398163$ & $-0,630036755$ & 0,630036755 & 0,4539905 & joint \\
\hline 50 & 43 & 281 & 0,820304748 & 1,762782545 & 0,717916674 & $-0,139548865$ & 0,68199836 & joint \\
\hline 50 & 47 & 175 & 0,750491578 & $-0,087266463$ & $-0,059440074$ & 0,67940315 & 0,731353702 & joint \\
\hline 50 & 49 & 251 & 0,715584993 & 1,239183769 & 0,620315999 & 0,213591927 & 0,75470958 & foliation \\
\hline 50 & 40 & 256 & 0,872664626 & 1,326450232 & 0,743289649 & 0,185322924 & 0,64278761 & foliation \\
\hline 50 & 61 & 280 & 0,506145483 & 1,745329252 & 0,477444273 & $-0,084186307$ & 0,874619707 & foliation \\
\hline 50 & 49 & 280 & 0,715584993 & 1,745329252 & 0,646092018 & $-0,113923455$ & 0,75470958 & joint \\
\hline 50 & 67 & 28 & 0,401425728 & $-2,652900463$ & $-0,183437154$ & $-0,344995109$ & 0,920504853 & joint \\
\hline
\end{tabular}




\begin{tabular}{|c|c|c|c|c|c|c|c|c|}
\hline \multirow{2}{*}{ depth (meter) } & \multicolumn{2}{|c|}{ measures } & \multirow{2}{*}{$\theta$} & \multirow{2}{*}{$\phi$} & \multicolumn{3}{|c|}{ cosine director } & \multirow{2}{*}{$\begin{array}{l}\text { geological } \\
\text { structure }\end{array}$} \\
\hline & $\operatorname{dip}$ & dipdirection & & & $\mathbf{x}$ & $\mathbf{y}$ & $\mathbf{z}$ & \\
\hline 50 & 77 & 45 & 0,226892803 & $-2,35619449$ & $-0,159064416$ & $-0,159064416$ & 0,974370065 & joint \\
\hline 50 & 29 & 117 & 1,064650844 & $-1,099557429$ & $-0,779291865$ & 0,397069038 & 0,48480962 & joint \\
\hline 50 & 31 & 123 & 1,029744259 & $-0,994837674$ & $-0,718880987$ & 0,466846772 & 0,515038075 & joint \\
\hline 50 & 60 & 18 & 0,523598776 & $-2,827433388$ & $-0,154508497$ & \begin{tabular}{|l|}
$-0,475528258$ \\
\end{tabular} & 0,866025404 & joint \\
\hline 50 & 46 & 347 & 0,767944871 & 2,914699851 & 0,156264133 & $-0,676854321$ & 0,7193398 & joint \\
\hline 50 & 36 & 112 & 0,942477796 & $-1,186823891$ & $-0,750107495$ & 0,3030631 & 0,587785252 & joint \\
\hline 50 & 72 & 152 & 0,314159265 & $-0,488692191$ & $-0,145074691$ & 0,272845811 & 0,951056516 & joint \\
\hline 50 & 18 & 78 & 1,256637061 & $-1,780235837$ & $-0,93027365$ & $-0,197735768$ & 0,309016994 & joint \\
\hline 50 & 29 & 140 & 1,064650844 & $-0,698131701$ & $-0,562194711$ & 0,669997566 & 0,48480962 & joint \\
\hline 50 & 35 & 79 & 0,959931089 & $-1,762782545$ & $-0,804101914$ & $-0,156301579$ & 0,573576436 & joint \\
\hline 50 & 74 & 284 & 0,27925268 & 1,815142422 & 0,267449748 & $-0,066682712$ & 0,961261696 & joint \\
\hline 50 & 87 & 230 & 0,052359878 & 0,872664626 & 0,040091668 & 0,033640904 & 0,998629535 & joint \\
\hline 50 & 89 & 226 & 0,017453293 & 0,802851456 & 0,012554211 & 0,01212346 & 0,999847695 & joint \\
\hline 50 & 57 & 276 & 0,575958653 & 1,675516082 & 0,541655445 & $-0,056930281$ & 0,838670568 & joint \\
\hline 50 & 88 & 180 & 0,034906585 & 0 & 0 & 0,034899497 & 0,999390827 & joint \\
\hline 50 & 85 & 178 & 0,087266463 & $-0,034906585$ & $-0,003041692$ & 0,08710265 & 0,996194698 & joint \\
\hline 50 & 81 & 318 & 0,157079633 & 2,408554368 & 0,104675088 & $-0,116253463$ & 0,987688341 & joint \\
\hline 50 & 44 & 286 & 0,802851456 & 1,850049007 & 0,691473796 & $-0,19827692$ & 0,69465837 & foliation \\
\hline 50 & 20 & 247 & 1,221730476 & 1,169370599 & 0,864991618 & 0,367167158 & 0,342020143 & joint \\
\hline 50 & 74 & 148 & 0,27925268 & $-0,558505361$ & $-0,146065545$ & 0,233753735 & 0,961261696 & joint \\
\hline 50 & 70 & 138 & 0,34906585 & $-0,733038286$ & $-0,228856146$ & 0,2541705 & 0,939692621 & joint \\
\hline 50 & 69 & 235 & 0,366519143 & 0,959931089 & 0,293557838 & 0,205551411 & 0,933580426 & joint \\
\hline 50 & 41 & 110 & 0,855211333 & $-1,221730476$ & $-0,709195023$ & 0,258125879 & 0,656059029 & joint \\
\hline 50 & 87 & 27 & 0,052359878 & $-2,670353756$ & $-0,023760027$ & $-0,046631678$ & 0,998629535 & joint \\
\hline 50 & 85 & 339 & 0,087266463 & 2,775073511 & 0,031233825 & $-0,081366895$ & 0,996194698 & joint \\
\hline 50 & 28 & 131 & 1,082104136 & $-0,855211333$ & $-0,666369007$ & 0,57926574 & 0,469471563 & joint \\
\hline 50 & 52 & 294 & 0,663225116 & 1,989675347 & 0,562434744 & $-0,250412082$ & 0,788010754 & joint \\
\hline 50 & 42 & 281 & 0,837758041 & 1,762782545 & 0,729491162 & $-0,141798718$ & 0,669130606 & foliation \\
\hline 50 & 64 & 345 & 0,453785606 & 2,879793266 & 0,113458802 & $-0,423434012$ & 0,898794046 & joint \\
\hline 50 & 61 & 274 & 0,506145483 & 1,640609497 & 0,483628648 & $-0,03381861$ & 0,874619707 & foliation \\
\hline 50 & 55 & 275 & 0,610865238 & 1,658062789 & 0,571393805 & $-0,04999048$ & 0,819152044 & foliation \\
\hline 50 & 63 & 295 & 0,471238898 & 2,00712864 & 0,411455125 & $-0,191864676$ & 0,891006524 & foliation \\
\hline 50 & 65 & 275 & 0,436332313 & 1,658062789 & 0,421010072 & $-0,036833609$ & 0,906307787 & foliation \\
\hline 50 & 57 & 288 & 0,575958653 & 1,884955592 & 0,517982503 & $-0,168302718$ & 0,838670568 & foliation \\
\hline 50 & 65 & 215 & 0,436332313 & 0,610865238 & 0,242403877 & 0,346188613 & 0,906307787 & joint \\
\hline 50 & 78 & 296 & 0,20943951 & 2,024581932 & 0,18686979 & $-0,091142486$ & 0,978147601 & joint \\
\hline 50 & 54 & 32 & 0,628318531 & $-2,583087293$ & $-0,311478728$ & $-0,498470164$ & 0,809016994 & joint \\
\hline 50 & 83 & 254 & 0,122173048 & 1,291543646 & 0,117148332 & 0,033591744 & 0,992546152 & joint \\
\hline 50 & 89 & 208 & 0,017453293 & 0,488692191 & 0,008193409 & 0,01540956 & 0,999847695 & joint \\
\hline 50 & 88 & 211 & 0,034906585 & 0,541052068 & 0,01797457 & 0,029914707 & 0,999390827 & joint \\
\hline 50 & 77 & 205 & 0,226892803 & 0,436332313 & 0,095068424 & 0,203874892 & 0,974370065 & joint \\
\hline 50 & 64 & 22 & 0,453785606 & $-2,757620218$ & $-0,164216722$ & $-0,40645065$ & 0,898794046 & joint \\
\hline 50 & 84 & 240 & 0,104719755 & 1,047197551 & 0,090524305 & 0,052264232 & 0,994521895 & joint \\
\hline 50 & 85 & 153 & 0,087266463 & $-0,471238898$ & $-0,039567879$ & 0,077656335 & 0,996194698 & joint \\
\hline 50 & 29 & 140 & 1,064650844 & $-0,698131701$ & $-0,562194711$ & 0,669997566 & 0,48480962 & joint \\
\hline 50 & 81 & 185 & 0,157079633 & 0,087266463 & 0,013634162 & 0,155839185 & 0,987688341 & joint \\
\hline 50 & 62 & 291 & 0,488692191 & 1,93731547 & 0,438289462 & $-0,168243561$ & 0,882947593 & joint \\
\hline 50 & 59 & 278 & 0,541052068 & 1,710422667 & 0,51002576 & $-0,071679446$ & 0,857167301 & joint \\
\hline 50 & 56 & 284 & 0,593411946 & 1,815142422 & 0,542582484 & $-0,135281007$ & 0,829037573 & joint \\
\hline 50 & 63 & 286 & 0,471238898 & 1,850049007 & 0,436403678 & $-0,125136741$ & 0,891006524 & joint \\
\hline 50 & 58 & 292 & 0,558505361 & 1,954768762 & 0,491332586 & $-0,19851125$ & 0,848048096 & joint \\
\hline 50 & 42 & 270 & 0,837758041 & 1,570796327 & 0,743144825 & $4,55231 \mathrm{E}-17$ & 0,669130606 & foliation \\
\hline 50 & 43 & 277 & 0,820304748 & 1,692969374 & 0,725902302 & $-0,089129595$ & 0,68199836 & foliation \\
\hline 50 & 72 & 119 & 0,314159265 & $-1,064650844$ & $-0,270272353$ & 0,149814412 & 0,951056516 & joint \\
\hline 50 & 81 & 265 & 0,157079633 & 1,483529864 & 0,155839185 & 0,013634162 & 0,987688341 & joint \\
\hline 50 & 78 & 261 & 0,20943951 & 1,413716694 & 0,205351953 & 0,032524554 & 0,978147601 & joint \\
\hline 50 & 41 & 275 & 0,855211333 & 1,658062789 & 0,751837682 & $-0,065777274$ & 0,656059029 & foliation \\
\hline
\end{tabular}




\begin{tabular}{|c|c|c|c|c|c|c|c|c|}
\hline \multirow{2}{*}{ depth (meter) } & \multicolumn{2}{|c|}{ measures } & \multirow{2}{*}{$\boldsymbol{\theta}$} & \multirow{2}{*}{$\phi$} & \multicolumn{3}{|c|}{ cosine director } & \multirow{2}{*}{$\begin{array}{l}\text { geological } \\
\text { structure }\end{array}$} \\
\hline & $\operatorname{dip}$ & dipdirection & & & $\mathbf{x}$ & $\mathbf{y}$ & $\mathbf{z}$ & \\
\hline 50 & 69 & \begin{tabular}{|l|}
266 \\
\end{tabular} & 0,366519143 & 1,500983157 & 0,357494983 & 0,024998484 & 0,933580426 & joint \\
\hline 50 & 78 & 217 & 0,20943951 & 0,645771823 & 0,125124379 & 0,166045659 & 0,978147601 & joint \\
\hline 50 & 36 & 275 & 0,942477796 & 1,658062789 & 0,80593844 & $-0,070510477$ & 0,587785252 & joint \\
\hline 50 & 41 & 263 & 0,855211333 & 1,448623279 & 0,749084089 & 0,091975961 & 0,656059029 & joint \\
\hline 50 & 54 & 268 & 0,628318531 & 1,535889742 & 0,587427189 & 0,020513409 & 0,809016994 & joint \\
\hline $51-60$ & 46 & 279 & 0,767944871 & 1,727875959 & 0,686105973 & $-0,108668511$ & 0,7193398 & foliation \\
\hline 60 & 88 & 208 & 0,034906585 & 0,488692191 & 0,016384321 & 0,030814427 & 0,999390827 & joint \\
\hline 60 & 57 & 274 & 0,575958653 & 1,640609497 & 0,543312322 & $-0,037992099$ & 0,838670568 & foliation \\
\hline 60 & 57 & 288 & 0,575958653 & 1,884955592 & 0,517982503 & $-0,168302718$ & 0,838670568 & foliation \\
\hline 60 & 57 & 277 & 0,575958653 & 1,692969374 & 0,540579378 & $-0,066374802$ & 0,838670568 & foliation \\
\hline 60 & 59 & 268 & 0,541052068 & 1,535889742 & 0,514724328 & 0,01797457 & 0,857167301 & foliation \\
\hline 60 & 53 & 270 & 0,645771823 & 1,570796327 & 0,601815023 & $3,68656 \mathrm{E}-17$ & 0,79863551 & foliation \\
\hline 60 & 79 & 215 & 0,191986218 & 0,610865238 & 0,109443544 & 0,156301579 & 0,981627183 & joint \\
\hline 60 & 28 & 119 & 1,082104136 & $-1,064650844$ & $-0,772243365$ & 0,428061487 & 0,469471563 & joint \\
\hline 60 & 21 & 115 & 1,204277184 & $-1,134464014$ & $-0,84611121$ & 0,394548137 & 0,35836795 & joint \\
\hline 60 & 32 & 21 & 1,012290966 & $-2,775073511$ & $-0,303913257$ & $-0,791721103$ & 0,529919264 & joint \\
\hline 60 & 29 & 120 & 1,064650844 & $-1,047197551$ & $-0,757442885$ & 0,437309854 & 0,48480962 & joint \\
\hline 60 & 76 & 42 & 0,244346095 & $-2,408554368$ & $-0,161877345$ & $-0,179783005$ & 0,970295726 & joint \\
\hline 60 & 24 & 247 & 1,151917306 & 1,169370599 & 0,840923028 & 0,356950648 & 0,406736643 & joint \\
\hline 60 & 63 & 54 & 0,471238898 & $-2,199114858$ & $-0,36728603$ & $-0,26684892$ & 0,891006524 & joint \\
\hline 60 & 71 & 53 & 0,331612558 & $-2,21656815$ & $-0,260010289$ & $-0,195931806$ & 0,945518576 & joint \\
\hline 60 & 66 & 63 & 0,41887902 & $-2,042035225$ & $-0,362405003$ & $-0,184654572$ & 0,913545458 & joint \\
\hline 60 & 87 & 230 & 0,052359878 & 0,872664626 & 0,040091668 & 0,033640904 & 0,998629535 & joint \\
\hline 60 & 68 & 48 & 0,383972435 & $-2,303834613$ & $-0,278386951$ & $-0,250660737$ & 0,927183855 & joint \\
\hline 60 & 92 & 276 & $-0,034906585$ & 1,675516082 & $-0,034708314$ & 0,003647991 & 0,999390827 & joint \\
\hline 60 & 87 & 229 & 0,052359878 & 0,855211333 & 0,039498448 & 0,034335477 & 0,998629535 & joint \\
\hline 60 & 78 & 213 & 0,20943951 & 0,575958653 & 0,113236823 & 0,174369416 & 0,978147601 & joint \\
\hline 60 & 44 & 280 & 0,802851456 & 1,745329252 & 0,708411412 & $-0,124912045$ & 0,69465837 & foliation \\
\hline 60 & 42 & 273 & 0,837758041 & 1,623156204 & 0,742126371 & $-0,038893195$ & 0,669130606 & foliation \\
\hline 60 & 47 & 264 & 0,750491578 & 1,466076572 & 0,678262302 & 0,071288241 & 0,731353702 & foliation \\
\hline 60 & 44 & 263 & 0,802851456 & 1,448623279 & 0,713977951 & 0,087665469 & 0,69465837 & foliation \\
\hline 60 & 38 & 289 & 0,907571211 & 1,902408885 & 0,745078805 & $-0,256551207$ & 0,615661475 & foliation \\
\hline 60 & 47 & 263 & 0,750491578 & 1,448623279 & 0,676914848 & 0,083114692 & 0,731353702 & foliation \\
\hline 60 & 63 & 267 & 0,471238898 & 1,518436449 & 0,453368322 & 0,023760027 & 0,891006524 & joint \\
\hline 60 & 45 & 263 & 0,785398163 & 1,448623279 & 0,701836114 & 0,086174639 & 0,707106781 & joint \\
\hline 60 & 27 & 268 & 1,099557429 & 1,535889742 & 0,890463747 & 0,031095679 & 0,4539905 & joint \\
\hline 60 & 40 & 282 & 0,872664626 & 1,780235837 & 0,749304534 & $-0,159269595$ & 0,64278761 & joint \\
\hline 60 & 43 & 287 & 0,820304748 & 1,8675023 & 0,699397023 & $-0,213827128$ & 0,68199836 & foliation \\
\hline 60 & 43 & 281 & 0,820304748 & 1,762782545 & 0,717916674 & $-0,139548865$ & 0,68199836 & foliation \\
\hline 60 & 84 & 238 & 0,104719755 & 1,012290966 & 0,088645164 & 0,055391646 & 0,994521895 & joint \\
\hline 60 & 68 & 22 & 0,383972435 & $-2,757620218$ & $-0,1403301$ & $-0,347329185$ & 0,927183855 & joint \\
\hline 60 & 85 & 347 & 0,087266463 & 2,914699851 & 0,019605776 & $-0,084921947$ & 0,996194698 & joint \\
\hline 60 & 45 & 268 & 0,785398163 & 1,535889742 & 0,706676031 & 0,024677671 & 0,707106781 & joint \\
\hline 60 & 60 & 286 & 0,523598776 & 1,850049007 & 0,480630848 & $-0,137818678$ & 0,866025404 & foliation \\
\hline 60 & 58 & 284 & 0,558505361 & 1,815142422 & 0,514178397 & $-0,128199073$ & 0,848048096 & foliation \\
\hline 60 & 53 & 286 & 0,645771823 & 1,850049007 & 0,57850173 & $-0,165882702$ & 0,79863551 & foliation \\
\hline 60 & 54 & 277 & 0,628318531 & 1,692969374 & 0,58340399 & $-0,071633003$ & 0,809016994 & foliation \\
\hline 60 & 74 & 260 & 0,27925268 & 1,396263402 & 0,271449805 & 0,047863925 & 0,961261696 & foliation \\
\hline 60 & 84 & 272 & 0,104719755 & 1,605702912 & 0,104464787 & $-0,003647991$ & 0,994521895 & foliation \\
\hline 60 & 59 & 268 & 0,541052068 & 1,535889742 & 0,514724328 & 0,01797457 & 0,857167301 & foliation \\
\hline 60 & 80 & 277 & 0,174532925 & 1,692969374 & 0,17235383 & $-0,021162389$ & 0,984807753 & joint \\
\hline 60 & 88 & 280 & 0,034906585 & 1,745329252 & 0,034369295 & $-0,006060234$ & 0,999390827 & joint \\
\hline 60 & 65 & 44 & 0,436332313 & $-2,373647783$ & $-0,293575313$ & $-0,304006136$ & 0,906307787 & joint \\
\hline 60 & 30 & 115 & 1,047197551 & $-1,134464014$ & $-0,784885567$ & 0,365998151 & 0,5 & joint \\
\hline 60 & 83 & 204 & 0,122173048 & 0,41887902 & 0,049568728 & 0,111333185 & 0,992546152 & joint \\
\hline 60 & 27 & 247 & 1,099557429 & 1,169370599 & 0,82017583 & 0,348143985 & 0,4539905 & joint \\
\hline 60 & 43 & 265 & 0,820304748 & 1,483529864 & 0,72857068 & 0,063741675 & 0,68199836 & foliation \\
\hline
\end{tabular}




\begin{tabular}{|c|c|c|c|c|c|c|c|c|}
\hline \multirow{2}{*}{ depth (meter) } & \multicolumn{2}{|c|}{ measures } & \multirow{2}{*}{$\boldsymbol{\theta}$} & \multirow{2}{*}{$\phi$} & \multicolumn{3}{|c|}{ cosine director } & \multirow{2}{*}{$\begin{array}{l}\text { geological } \\
\text { structure }\end{array}$} \\
\hline & $\operatorname{dip}$ & dipdirection & & & $\mathbf{x}$ & \begin{tabular}{|c|}
$\mathbf{y}$ \\
\end{tabular} & $\mathbf{z}$ & \\
\hline 60 & 40 & 271 & 0,872664626 & 1,588249619 & 0,765927771 & \begin{tabular}{|l|}
$-0,013369319$ \\
\end{tabular} & 0,64278761 & foliation \\
\hline 60 & 86 & 356 & 0,06981317 & 3,071779484 & 0,004865966 & $-0,06958655$ & 0,99756405 & joint \\
\hline 60 & 71 & 143 & 0,331612558 & $-0,645771823$ & $-0,195931806$ & 0,260010289 & 0,945518576 & joint \\
\hline 60 & 41 & 285 & 0,855211333 & 1,832595715 & 0,728993475 & \begin{tabular}{|l|}
$-0,195333213$ \\
\end{tabular} & 0,656059029 & foliation \\
\hline 60 & 46 & 180 & 0,767944871 & 0 & 0 & \begin{tabular}{|c|}
0,69465837 \\
\end{tabular} & 0,7193398 & joint \\
\hline 60 & 51 & 266 & 0,680678408 & 1,500983157 & 0,627787398 & 0,043899171 & 0,777145961 & joint \\
\hline 60 & 71 & 10 & 0,331612558 & $-2,967059728$ & $-0,056534317$ & \begin{tabular}{|l|}
$-0,320622043$ \\
\end{tabular} & 0,945518576 & joint \\
\hline 60 & 40 & 279 & 0,872664626 & 1,727875959 & 0,756613165 & $-0,119835753$ & 0,64278761 & joint \\
\hline 60 & 42 & 271 & 0,837758041 & 1,588249619 & 0,743031641 & \begin{tabular}{|l|}
$-0,012969666$ \\
\end{tabular} & 0,669130606 & joint \\
\hline 60 & 43 & 283 & 0,820304748 & 1,79768913 & 0,712609154 & \begin{tabular}{|c|}
$-0,164518786$ \\
\end{tabular} & 0,68199836 & joint \\
\hline 60 & 63 & 272 & 0,471238898 & 1,605702912 & 0,453713941 & \begin{tabular}{|c|}
$-0,01584404$ \\
\end{tabular} & 0,891006524 & foliation \\
\hline 60 & 57 & 275 & 0,575958653 & 1,658062789 & 0,542566519 & $-0,04746842$ & 0,838670568 & foliation \\
\hline 60 & 54 & 276 & 0,628318531 & 1,675516082 & 0,584565303 & \begin{tabular}{|l|}
$-0,061440289$ \\
\end{tabular} & 0,809016994 & foliation \\
\hline 60 & 55 & 275 & 0,610865238 & 1,658062789 & 0,571393805 & $-0,04999048$ & 0,819152044 & foliation \\
\hline 60 & 58 & 284 & 0,558505361 & 1,815142422 & 0,514178397 & \begin{tabular}{|l|}
$-0,128199073$ \\
\end{tabular} & 0,848048096 & foliation \\
\hline 60 & 84 & 143 & 0,104719755 & $-0,645771823$ & $-0,0629068$ & 0,083480143 & 0,994521895 & joint \\
\hline 60 & 63 & 218 & 0,471238898 & 0,663225116 & 0,279504461 & 0,357749396 & 0,891006524 & joint \\
\hline 60 & 51 & 277 & 0,680678408 & 1,692969374 & 0,624629532 & \begin{tabular}{|l|}
$-0,076694863$ \\
\end{tabular} & 0,777145961 & joint \\
\hline 60 & 85 & 227 & 0,087266463 & 0,820304748 & 0,063741675 & 0,059440074 & 0,996194698 & joint \\
\hline 60 & 60 & 275 & 0,523598776 & 1,658062789 & 0,498097349 & \begin{tabular}{|l|}
$-0,043577871$ \\
\end{tabular} & 0,866025404 & joint \\
\hline 60 & 41 & 253 & 0,855211333 & 1,274090354 & 0,721732361 & 0,220655727 & 0,656059029 & joint \\
\hline 60 & 50 & 268 & 0,698131701 & 1,535889742 & 0,642396041 & 0,022432964 & 0,766044443 & foliation \\
\hline 60 & 76 & 37 & 0,244346095 & $-2,49582083$ & $-0,145592231$ & $-0,193207416$ & 0,970295726 & joint \\
\hline 60 & 52 & 131 & 0,663225116 & $-0,855211333$ & $-0,464645614$ & \begin{tabular}{|c|}
0,40391027 \\
\end{tabular} & 0,788010754 & joint \\
\hline 60 & 66 & 225 & 0,41887902 & 0,785398163 & 0,287606238 & 0,287606238 & 0,913545458 & joint \\
\hline 60 & 47 & 283 & 0,750491578 & 1,79768913 & 0,664518786 & $-0,15341625$ & 0,731353702 & joint \\
\hline 60 & 70 & 210 & 0,34906585 & 0,523598776 & 0,171010072 & 0,296198133 & 0,939692621 & joint \\
\hline 60 & 88 & 168 & 0,034906585 & $-0,20943951$ & $-0,007256013$ & 0,034136859 & 0,999390827 & joint \\
\hline 60 & 70 & 210 & 0,34906585 & 0,523598776 & 0,171010072 & 0,296198133 & 0,939692621 & joint \\
\hline 60 & 45 & 185 & 0,785398163 & 0,087266463 & 0,061628417 & 0,704416026 & 0,707106781 & joint \\
\hline 60 & 37 & 268 & 0,925024504 & 1,535889742 & 0,798149003 & 0,027871977 & 0,601815023 & joint \\
\hline 60 & 88 & 224 & 0,034906585 & 0,767944871 & 0,024243228 & 0,025104597 & 0,999390827 & joint \\
\hline 60 & 68 & 211 & 0,383972435 & 0,541052068 & 0,192936659 & 0,321100523 & 0,927183855 & joint \\
\hline 60 & 25 & 119 & 1,134464014 & $-1,064650844$ & $-0,792674651$ & 0,439386734 & 0,422618262 & joint \\
\hline 60 & 80 & 237 & 0,174532925 & 0,994837674 & 0,145633616 & 0,094575576 & 0,984807753 & joint \\
\hline 60 & 88 & 152 & 0,034906585 & $-0,488692191$ & $-0,016384321$ & 0,030814427 & 0,999390827 & joint \\
\hline 60 & 26 & 119 & 1,117010721 & $-1,064650844$ & $-0,786102986$ & 0,435744 & 0,438371147 & joint \\
\hline 60 & 54 & 276 & 0,628318531 & 1,675516082 & 0,584565303 & \begin{tabular}{|c|}
$-0,061440289$ \\
\end{tabular} & 0,809016994 & foliation \\
\hline 60 & 65 & 270 & 0,436332313 & 1,570796327 & 0,422618262 & \begin{tabular}{|l|}
$2,58885 \mathrm{E}-17$ \\
\end{tabular} & 0,906307787 & foliation \\
\hline 60 & 38 & 272 & 0,907571211 & 1,605702912 & 0,787530719 & \begin{tabular}{|l|}
$-0,027501179$ \\
\end{tabular} & 0,615661475 & joint \\
\hline 60 & 87 & 141 & 0,052359878 & $-0,680678408$ & $-0,032936084$ & 0,040672677 & 0,998629535 & joint \\
\hline 60 & 86 & 173 & 0,06981317 & $-0,122173048$ & $-0,008501176$ & 0,06923652 & 0,99756405 & joint \\
\hline surface & 44 & 282 & 0,802851456 & 1,780235837 & 0,7036205 & $-0,149559154$ & 0,69465837 & foliation \\
\hline surface & 49 & 284 & 0,715584993 & 1,815142422 & 0,636571272 & $-0,158715044$ & 0,75470958 & foliation \\
\hline surface & 51 & 278 & 0,680678408 & 1,710422667 & 0,623195888 & \begin{tabular}{|c|}
$-0,08758447$ \\
\end{tabular} & 0,777145961 & foliation \\
\hline surface & 44 & 287 & 0,802851456 & 1,8675023 & 0,687908072 & \begin{tabular}{|l|}
$-0,210314604$ \\
\end{tabular} & 0,69465837 & foliation \\
\hline surface & 55 & 283 & 0,610865238 & 1,79768913 & 0,558875709 & \begin{tabular}{|l|}
$-0,129026624$ \\
\end{tabular} & 0,819152044 & foliation \\
\hline surface & 57 & 284 & 0,575958653 & 1,815142422 & 0,528460928 & \begin{tabular}{|l|}
$-0,131760108$ \\
\end{tabular} & 0,838670568 & foliation \\
\hline surface & 63 & 282 & 0,471238898 & 1,780235837 & 0,444069718 & \begin{tabular}{|l|}
$-0,094389932$ \\
\end{tabular} & 0,891006524 & foliation \\
\hline surface & 57 & 274 & 0,575958653 & 1,640609497 & 0,543312322 & \begin{tabular}{|l|}
$-0,037992099$ \\
\end{tabular} & 0,838670568 & foliation \\
\hline surface & 47 & 271 & 0,750491578 & 1,588249619 & 0,681894488 & \begin{tabular}{|l|}
$-0,011902513$ \\
\end{tabular} & 0,731353702 & foliation \\
\hline surface & 47 & 275 & 0,750491578 & 1,658062789 & 0,67940315 & $-0,059440074$ & 0,731353702 & foliation \\
\hline surface & 43 & 285 & 0,820304748 & 1,832595715 & 0,706433429 & $-0,189288267$ & 0,68199836 & foliation \\
\hline surface & 47 & 283 & 0,750491578 & 1,79768913 & 0,664518786 & $-0,15341625$ & 0,731353702 & foliation \\
\hline surface & 57 & 282 & 0,575958653 & 1,780235837 & 0,532737365 & \begin{tabular}{|l|}
$-0,113236823$ \\
\end{tabular} & 0,838670568 & foliation \\
\hline surface & 43 & 279 & 0,820304748 & 1,727875959 & 0,722349524 & $-0,114408925$ & 0,68199836 & foliation \\
\hline surface & 53 & 268 & 0,645771823 & 1,535889742 & 0,601448414 & 0,021003041 & 0,79863551 & foliation \\
\hline
\end{tabular}




\begin{tabular}{|c|c|c|c|c|c|c|c|c|}
\hline \multirow{2}{*}{ depth (meter) } & \multicolumn{2}{|c|}{ measures } & \multirow{2}{*}{$\theta$} & \multirow{2}{*}{$\phi$} & \multicolumn{3}{|c|}{ cosine director } & \multirow{2}{*}{$\begin{array}{l}\text { geological } \\
\text { structure }\end{array}$} \\
\hline & dip & dipdirection & & & $\mathbf{x}$ & \begin{tabular}{|c|}
$\mathbf{y}$ \\
\end{tabular} & $\mathbf{z}$ & \\
\hline surface & 48 & 277 & 0,733038286 & 1,692969374 & 0,664143008 & $-0,081546508$ & 0,743144825 & foliation \\
\hline surface & 52 & 274 & 0,663225116 & 1,640609497 & 0,614161755 & $-0,042946374$ & 0,788010754 & foliation \\
\hline surface & 55 & 273 & 0,610865238 & 1,623156204 & 0,57279037 & $-0,030018671$ & 0,819152044 & foliation \\
\hline surface & 61 & 272 & 0,506145483 & 1,605702912 & 0,484514287 & $-0,016919612$ & 0,874619707 & foliation \\
\hline surface & 44 & 278 & 0,802851456 & 1,710422667 & 0,712339235 & $-0,100112751$ & 0,69465837 & foliation \\
\hline surface & 59 & 277 & 0,541052068 & 1,692969374 & 0,511199059 & $-0,062767352$ & 0,857167301 & foliation \\
\hline surface & 56 & 277 & 0,593411946 & 1,692969374 & 0,555024764 & $-0,068148472$ & 0,829037573 & foliation \\
\hline surface & 44 & 288 & 0,802851456 & 1,884955592 & 0,684132805 & $-0,222288223$ & 0,69465837 & foliation \\
\hline surface & 48 & 284 & 0,733038286 & 1,815142422 & 0,649254568 & $-0,161877345$ & 0,743144825 & foliation \\
\hline surface & 56 & 278 & 0,593411946 & 1,710422667 & 0,553750877 & $-0,07782461$ & 0,829037573 & foliation \\
\hline surface & 47 & 274 & 0,750491578 & 1,640609497 & 0,680337046 & $-0,047573801$ & 0,731353702 & foliation \\
\hline surface & 42 & 272 & 0,837758041 & 1,605702912 & 0,742692122 & $-0,02593538$ & 0,669130606 & foliation \\
\hline surface & 44 & 278 & 0,802851456 & 1,710422667 & 0,712339235 & $-0,100112751$ & 0,69465837 & foliation \\
\hline surface & 46 & 278 & 0,767944871 & 1,710422667 & 0,687898003 & $-0,09667776$ & 0,7193398 & foliation \\
\hline surface & 62 & 284 & 0,488692191 & 1,815142422 & 0,455526251 & $-0,11357545$ & 0,882947593 & foliation \\
\hline surface & 54 & 284 & 0,628318531 & 1,815142422 & 0,570325518 & $-0,142198122$ & 0,809016994 & foliation \\
\hline surface & 43 & 273 & 0,820304748 & 1,623156204 & 0,730351407 & $-0,038276095$ & 0,68199836 & foliation \\
\hline surface & 53 & 278 & 0,645771823 & 1,710422667 & 0,595958201 & $-0,083756463$ & 0,79863551 & foliation \\
\hline surface & 36 & 284 & 0,942477796 & 1,815142422 & 0,784985732 & $-0,195718925$ & 0,587785252 & foliation \\
\hline surface & 34 & 283 & 0,977384381 & 1,79768913 & 0,807789393 & $-0,186492876$ & 0,559192903 & foliation \\
\hline surface & 43 & 277 & 0,820304748 & 1,692969374 & 0,725902302 & $-0,089129595$ & 0,68199836 & foliation \\
\hline surface & 49 & 282 & 0,715584993 & 1,780235837 & 0,641722565 & $-0,136402342$ & 0,75470958 & foliation \\
\hline surface & 47 & 265 & 0,750491578 & 1,483529864 & 0,67940315 & 0,059440074 & 0,731353702 & foliation \\
\hline surface & 40 & 266 & 0,872664626 & 1,500983157 & 0,764178397 & 0,053436559 & 0,64278761 & foliation \\
\hline surface & 63 & 269 & 0,471238898 & 1,553343034 & 0,453921355 & 0,007923227 & 0,891006524 & foliation \\
\hline surface & 43 & 276 & 0,820304748 & 1,675516082 & 0,72734727 & $-0,076447279$ & 0,68199836 & foliation \\
\hline surface & 42 & 275 & 0,837758041 & 1,658062789 & 0,740316935 & $-0,064769339$ & 0,669130606 & foliation \\
\hline surface & 59 & 287 & 0,541052068 & 1,8675023 & 0,492533361 & $-0,15058256$ & 0,857167301 & foliation \\
\hline surface & 40 & 267 & 0,872664626 & 1,518436449 & 0,764994606 & 0,040091668 & 0,64278761 & foliation \\
\hline surface & 57 & 280 & 0,575958653 & 1,745329252 & 0,536364744 & $-0,094575576$ & 0,838670568 & foliation \\
\hline surface & 40 & 279 & 0,872664626 & 1,727875959 & 0,756613165 & $-0,119835753$ & 0,64278761 & foliation \\
\hline surface & 38 & 273 & 0,907571211 & 1,623156204 & 0,786930812 & $-0,041241296$ & 0,615661475 & foliation \\
\hline surface & 72 & 283 & 0,314159265 & 1,79768913 & 0,301096909 & $-0,069513699$ & 0,951056516 & foliation \\
\hline surface & 50 & 272 & 0,698131701 & 1,605702912 & 0,642396041 & $-0,022432964$ & 0,766044443 & foliation \\
\hline surface & 51 & 273 & 0,680678408 & 1,623156204 & 0,628457929 & $-0,032936084$ & 0,777145961 & foliation \\
\hline surface & 75 & 263 & 0,261799388 & 1,448623279 & 0,256889847 & 0,031542107 & 0,965925826 & foliation \\
\hline surface & 45 & 280 & 0,785398163 & 1,745329252 & 0,69636424 & $-0,122787804$ & 0,707106781 & foliation \\
\hline surface & 41 & 291 & 0,855211333 & 1,93731547 & 0,704582092 & $-0,270463725$ & 0,656059029 & foliation \\
\hline surface & 42 & 290 & 0,837758041 & 1,919862177 & 0,698327709 & $-0,2541705$ & 0,669130606 & foliation \\
\hline surface & 45 & 280 & 0,785398163 & 1,745329252 & 0,69636424 & $-0,122787804$ & 0,707106781 & foliation \\
\hline surface & 40 & 282 & 0,872664626 & 1,780235837 & 0,749304534 & $-0,159269595$ & 0,64278761 & foliation \\
\hline surface & 40 & 280 & 0,872664626 & 1,745329252 & 0,754406507 & $-0,133022222$ & 0,64278761 & foliation \\
\hline surface & 50 & 288 & 0,698131701 & 1,884955592 & 0,611327345 & $-0,198632295$ & 0,766044443 & foliation \\
\hline surface & 52 & 285 & 0,663225116 & 1,832595715 & 0,594683319 & $-0,159344915$ & 0,788010754 & foliation \\
\hline surface & 58 & 277 & 0,558505361 & 1,692969374 & 0,525969326 & $-0,064580913$ & 0,848048096 & foliation \\
\hline surface & 52 & 270 & 0,663225116 & 1,570796327 & 0,615661475 & $3,77138 \mathrm{E}-17$ & 0,788010754 & foliation \\
\hline surface & 52 & 274 & 0,663225116 & 1,640609497 & 0,614161755 & $-0,042946374$ & 0,788010754 & foliation \\
\hline surface & 49 & 270 & 0,715584993 & 1,570796327 & 0,656059029 & $4,01885 \mathrm{E}-17$ & 0,75470958 & foliation \\
\hline surface & 46 & 281 & 0,767944871 & 1,762782545 & 0,68189554 & $-0,132547066$ & 0,7193398 & foliation \\
\hline surface & 48 & 274 & 0,733038286 & 1,640609497 & 0,667500638 & $-0,046676192$ & 0,743144825 & foliation \\
\hline surface & 44 & 272 & 0,802851456 & 1,605702912 & 0,718901598 & $-0,025104597$ & 0,69465837 & foliation \\
\hline surface & 55 & 282 & 0,610865238 & 1,780235837 & 0,561042415 & $-0,119253247$ & 0,819152044 & foliation \\
\hline surface & 56 & 284 & 0,593411946 & 1,815142422 & 0,542582484 & $-0,135281007$ & 0,829037573 & foliation \\
\hline surface & 48 & 276 & 0,733038286 & 1,675516082 & 0,665465039 & $-0,069943194$ & 0,743144825 & foliation \\
\hline surface & 53 & 270 & 0,645771823 & 1,570796327 & 0,601815023 & $3,68656 \mathrm{E}-17$ & 0,79863551 & foliation \\
\hline surface & 58 & 281 & 0,558505361 & 1,762782545 & 0,520183155 & $-0,101113362$ & 0,848048096 & foliation \\
\hline surface & 54 & 278 & 0,628318531 & 1,710422667 & 0,582064967 & $-0,081803896$ & 0,809016994 & foliation \\
\hline
\end{tabular}




\begin{tabular}{|c|c|c|c|c|c|c|c|c|}
\hline \multirow{2}{*}{ depth (meter) } & \multicolumn{2}{|c|}{ measures } & \multirow{2}{*}{$\boldsymbol{\theta}$} & \multirow{2}{*}{$\phi$} & \multicolumn{3}{|c|}{ cosine director } & \multirow{2}{*}{$\begin{array}{l}\text { geological } \\
\text { structure }\end{array}$} \\
\hline & dip & dipdirection & & & $\mathbf{x}$ & \begin{tabular}{|c|}
$\mathbf{y}$ \\
\end{tabular} & $\mathbf{z}$ & \\
\hline surface & 53 & 287 & 0,645771823 & 1,8675023 & 0,575518569 & $-0,175953684$ & 0,79863551 & foliation \\
\hline surface & 36 & 273 & 0,942477796 & 1,623156204 & 0,807908265 & $-0,042340678$ & 0,587785252 & foliation \\
\hline surface & 41 & 279 & 0,855211333 & 1,727875959 & 0,745417853 & $-0,118062589$ & 0,656059029 & foliation \\
\hline surface & 57 & 269 & 0,575958653 & 1,553343034 & 0,544556084 & 0,009505262 & 0,838670568 & foliation \\
\hline surface & 37 & 275 & 0,925024504 & 1,658062789 & 0,795596461 & $-0,069605671$ & 0,601815023 & foliation \\
\hline surface & 55 & 272 & 0,610865238 & 1,605702912 & 0,573227029 & $-0,020017529$ & 0,819152044 & foliation \\
\hline surface & 39 & 280 & 0,890117919 & 1,745329252 & 0,765339368 & $-0,13494998$ & 0,629320391 & foliation \\
\hline surface & 31 & 266 & 1,029744259 & 1,500983157 & 0,855079284 & 0,059792968 & 0,515038075 & foliation \\
\hline surface & 43 & 276 & 0,820304748 & 1,675516082 & 0,72734727 & $-0,076447279$ & 0,68199836 & foliation \\
\hline surface & 38 & 275 & 0,907571211 & 1,658062789 & 0,785012135 & $-0,068679663$ & 0,615661475 & foliation \\
\hline surface & 53 & 254 & 0,645771823 & 1,291543646 & 0,57850173 & 0,165882702 & 0,79863551 & foliation \\
\hline surface & 31 & 277 & 1,029744259 & 1,692969374 & 0,850778106 & $-0,104462416$ & 0,515038075 & foliation \\
\hline surface & 43 & 267 & 0,820304748 & 1,518436449 & 0,730351407 & 0,038276095 & 0,68199836 & foliation \\
\hline surface & 40 & 268 & 0,872664626 & 1,535889742 & 0,76557779 & 0,026734566 & 0,64278761 & foliation \\
\hline surface & 59 & 271 & 0,541052068 & 1,588249619 & 0,514959632 & $-0,008988654$ & 0,857167301 & foliation \\
\hline surface & 89 & 276 & 0,017453293 & 1,675516082 & 0,0173568 & $-0,001824273$ & 0,999847695 & foliation \\
\hline surface & 49 & 262 & 0,715584993 & 1,431169987 & 0,649674308 & 0,091305769 & 0,75470958 & foliation \\
\hline surface & 53 & 266 & 0,645771823 & 1,500983157 & 0,600349032 & 0,041980494 & 0,79863551 & foliation \\
\hline surface & 86 & 249 & 0,06981317 & 1,204277184 & 0,065123279 & 0,024998484 & 0,99756405 & foliation \\
\hline surface & 57 & 270 & 0,575958653 & 1,570796327 & 0,544639035 & $3,33632 \mathrm{E}-17$ & 0,838670568 & foliation \\
\hline surface & 50 & 277 & 0,698131701 & 1,692969374 & 0,637996368 & $-0,078336104$ & 0,766044443 & foliation \\
\hline surface & 34 & 95 & 0,977384381 & $-1,483529864$ & $-0,825882834$ & 0,072255385 & 0,559192903 & foliation \\
\hline surface & 25 & 120 & 1,134464014 & $-1,047197551$ & $-0,784885567$ & 0,453153894 & 0,422618262 & foliation \\
\hline surface & 60 & 120 & 0,523598776 & $-1,047197551$ & $-0,433012702$ & 0,25 & 0,866025404 & foliation \\
\hline surface & 15 & 130 & 1,308996939 & $-0,872664626$ & $-0,739942112$ & 0,620885153 & 0,258819045 & foliation \\
\hline surface & 42 & 145 & 0,837758041 & $-0,610865238$ & $-0,426250361$ & 0,608748603 & 0,669130606 & foliation \\
\hline surface & 10 & 190 & 1,396263402 & 0,174532925 & 0,171010072 & 0,96984631 & 0,173648178 & foliation \\
\hline surface & 20 & 195 & 1,221730476 & 0,261799388 & 0,243210347 & 0,907673371 & 0,342020143 & foliation \\
\hline surface & 5 & 225 & 1,483529864 & 0,785398163 & 0,704416026 & 0,704416026 & 0,087155743 & foliation \\
\hline surface & 35 & 229 & 0,959931089 & 0,855211333 & 0,618221895 & 0,537412095 & 0,573576436 & foliation \\
\hline surface & 40 & 230 & 0,872664626 & 0,872664626 & 0,586824089 & 0,492403877 & 0,64278761 & foliation \\
\hline surface & 55 & 233 & 0,610865238 & 0,925024504 & 0,45807851 & 0,345186916 & 0,819152044 & foliation \\
\hline surface & 30 & 235 & 1,047197551 & 0,959931089 & 0,70940648 & 0,496731765 & 0,5 & foliation \\
\hline surface & 31 & 235 & 1,029744259 & 0,959931089 & 0,702150347 & 0,491650966 & 0,515038075 & foliation \\
\hline surface & 42 & 235 & 0,837758041 & 0,959931089 & 0,608748603 & 0,426250361 & 0,669130606 & foliation \\
\hline surface & 60 & 235 & 0,523598776 & 0,959931089 & 0,409576022 & 0,286788218 & 0,866025404 & foliation \\
\hline surface & 30 & 240 & 1,047197551 & 1,047197551 & 0,75 & 0,433012702 & 0,5 & foliation \\
\hline surface & 27 & 241 & 1,099557429 & 1,064650844 & 0,779291865 & 0,431968535 & 0,4539905 & foliation \\
\hline surface & 21 & 244 & 1,204277184 & 1,117010721 & 0,839096529 & 0,409254722 & 0,35836795 & foliation \\
\hline surface & 30 & 245 & 1,047197551 & 1,134464014 & 0,784885567 & 0,365998151 & 0,5 & foliation \\
\hline surface & 45 & 245 & 0,785398163 & 1,134464014 & 0,640856382 & 0,298836239 & 0,707106781 & foliation \\
\hline surface & 22 & 245 & 1,186823891 & 1,134464014 & 0,840313947 & 0,391844829 & 0,374606593 & foliation \\
\hline surface & 35 & 245 & 0,959931089 & 1,134464014 & 0,742403877 & 0,346188613 & 0,573576436 & foliation \\
\hline surface & 50 & 249 & 0,698131701 & 1,204277184 & 0,600093931 & 0,230354478 & 0,766044443 & foliation \\
\hline surface & 29 & 250 & 1,064650844 & 1,221730476 & 0,821873685 & 0,299137558 & 0,48480962 & foliation \\
\hline surface & 58 & 250 & 0,558505361 & 1,221730476 & 0,497961222 & 0,181243063 & 0,848048096 & foliation \\
\hline surface & 60 & 250 & 0,523598776 & 1,221730476 & 0,46984631 & 0,171010072 & 0,866025404 & foliation \\
\hline surface & 35 & 253 & 0,959931089 & 1,274090354 & 0,783358996 & 0,23949688 & 0,573576436 & foliation \\
\hline surface & 30 & 255 & 1,047197551 & 1,308996939 & 0,836516304 & 0,224143868 & 0,5 & foliation \\
\hline surface & 40 & 255 & 0,872664626 & 1,308996939 & 0,739942112 & 0,198266891 & 0,64278761 & foliation \\
\hline surface & 35 & 255 & 0,959931089 & 1,308996939 & 0,791240115 & 0,21201215 & 0,573576436 & foliation \\
\hline surface & 78 & 255 & 0,20943951 & 1,308996939 & 0,200827272 & 0,053811505 & 0,978147601 & foliation \\
\hline surface & 40 & 255 & 0,872664626 & 1,308996939 & 0,739942112 & 0,198266891 & 0,64278761 & foliation \\
\hline surface & 50 & 260 & 0,698131701 & 1,396263402 & 0,633022222 & 0,111618897 & 0,766044443 & foliation \\
\hline surface & 40 & 260 & 0,872664626 & 1,396263402 & 0,754406507 & 0,133022222 & 0,64278761 & foliation \\
\hline surface & 40 & 260 & 0,872664626 & 1,396263402 & 0,754406507 & 0,133022222 & 0,64278761 & foliation \\
\hline surface & 50 & 260 & 0,698131701 & 1,396263402 & 0,633022222 & 0,111618897 & 0,766044443 & foliation \\
\hline
\end{tabular}




\begin{tabular}{|c|c|c|c|c|c|c|c|c|}
\hline \multirow{2}{*}{ depth (meter) } & \multicolumn{2}{|c|}{ measures } & \multirow{2}{*}{$\boldsymbol{\theta}$} & \multirow{2}{*}{$\phi$} & \multicolumn{3}{|c|}{ cosine director } & \multirow{2}{*}{$\begin{array}{l}\text { geological } \\
\text { structure }\end{array}$} \\
\hline & dip & dipdirection & & & $\mathbf{x}$ & \begin{tabular}{|c|}
$\mathbf{y}$ \\
\end{tabular} & $\mathbf{z}$ & \\
\hline surface & 35 & 260 & 0,959931089 & 1,396263402 & 0,806707284 & 0,14224426 & 0,573576436 & foliation \\
\hline surface & 7 & 262 & 1,448623279 & 1,431169987 & 0,982886761 & 0,138135726 & 0,121869343 & foliation \\
\hline surface & 45 & 265 & 0,785398163 & 1,483529864 & 0,704416026 & 0,061628417 & 0,707106781 & foliation \\
\hline surface & 50 & 265 & 0,698131701 & 1,483529864 & 0,640341609 & 0,056022632 & 0,766044443 & foliation \\
\hline surface & 39 & 267 & 0,890117919 & 1,518436449 & 0,77608091 & 0,040672677 & 0,629320391 & foliation \\
\hline surface & 55 & 270 & 0,610865238 & 1,570796327 & 0,573576436 & $3,51358 \mathrm{E}-17$ & 0,819152044 & foliation \\
\hline surface & 51 & 270 & 0,680678408 & 1,570796327 & 0,629320391 & $3,85505 \mathrm{E}-17$ & 0,777145961 & foliation \\
\hline surface & 42 & 274 & 0,837758041 & 1,640609497 & 0,741334562 & $-0,051839163$ & 0,669130606 & foliation \\
\hline surface & 45 & 275 & 0,785398163 & 1,658062789 & 0,704416026 & $-0,061628417$ & 0,707106781 & foliation \\
\hline surface & 52 & 275 & 0,663225116 & 1,658062789 & 0,613318698 & $-0,053658433$ & 0,788010754 & foliation \\
\hline surface & 25 & 275 & 1,134464014 & 1,658062789 & 0,902859012 & $-0,078989928$ & 0,422618262 & foliation \\
\hline surface & 62 & 280 & 0,488692191 & 1,745329252 & 0,462339235 & $-0,081522881$ & 0,882947593 & foliation \\
\hline surface & 13 & 285 & 1,343903524 & 1,832595715 & 0,94116921 & $-0,25218553$ & 0,224951054 & foliation \\
\hline surface & 35 & 285 & 0,959931089 & 1,832595715 & 0,791240115 & $-0,21201215$ & 0,573576436 & foliation \\
\hline surface & 15 & 290 & 1,308996939 & 1,919862177 & 0,907673371 & $-0,33036609$ & 0,258819045 & foliation \\
\hline surface & 38 & 294 & 0,907571211 & 1,989675347 & 0,719883645 & $-0,320512849$ & 0,615661475 & foliation \\
\hline surface & 39 & 297 & 0,890117919 & 2,042035225 & 0,692442122 & $-0,352816883$ & 0,629320391 & foliation \\
\hline surface & 30 & 300 & 1,047197551 & 2,094395102 & 0,75 & $-0,433012702$ & 0,5 & foliation \\
\hline surface & 47 & 300 & 0,750491578 & 2,094395102 & 0,590627905 & $-0,34099918$ & 0,731353702 & foliation \\
\hline surface & 25 & 300 & 1,134464014 & 2,094395102 & 0,784885567 & $-0,453153894$ & 0,422618262 & foliation \\
\hline surface & 20 & 310 & 1,221730476 & 2,268928028 & 0,71984631 & $-0,604022774$ & 0,342020143 & foliation \\
\hline surface & 41 & 310 & 0,855211333 & 2,268928028 & 0,57814108 & $-0,485117967$ & 0,656059029 & foliation \\
\hline surface & 24 & 320 & 1,151917306 & 2,443460953 & 0,587215701 & $-0,699816421$ & 0,406736643 & foliation \\
\hline surface & 4 & 322 & 1,500983157 & 2,478367538 & 0,614161755 & $-0,786091199$ & 0,069756474 & foliation \\
\hline surface & 10 & 330 & 1,396263402 & 2,617993878 & 0,492403877 & $-0,852868532$ & 0,173648178 & foliation \\
\hline surface & 40 & 330 & 0,872664626 & 2,617993878 & 0,383022222 & $-0,663413948$ & 0,64278761 & foliation \\
\hline surface & 23 & 330 & 1,169370599 & 2,617993878 & 0,460252427 & $-0,797180587$ & 0,390731128 & foliation \\
\hline surface & 24 & 340 & 1,151917306 & 2,792526803 & 0,312450948 & $-0,858451925$ & 0,406736643 & foliation \\
\hline surface & 48 & 350 & 0,733038286 & 2,967059728 & 0,11619331 & $-0,658965009$ & 0,743144825 & foliation \\
\hline surface & 8 & 355 & 1,431169987 & 3,054326191 & 0,086307549 & $-0,9864998$ & 0,139173101 & foliation \\
\hline surface & 42 & 55 & 0,837758041 & $-2,181661565$ & $-0,608748603$ & $-0,426250361$ & 0,669130606 & foliation \\
\hline surface & 42 & 69 & 0,837758041 & $-1,93731547$ & $-0,693785463$ & $-0,266319287$ & 0,669130606 & foliation \\
\hline surface & 35 & 79 & 0,959931089 & $-1,762782545$ & $-0,804101914$ & $-0,156301579$ & 0,573576436 & foliation \\
\hline surface & 50 & 85 & 0,698131701 & $-1,658062789$ & $-0,640341609$ & $-0,056022632$ & 0,766044443 & foliation \\
\hline surface & 54 & 239 & 0,628318531 & 1,029744259 & 0,503830298 & 0,302731785 & 0,809016994 & joint \\
\hline surface & 59 & 270 & 0,541052068 & 1,570796327 & 0,515038075 & $3,15499 \mathrm{E}-17$ & 0,857167301 & joint \\
\hline surface & 88 & 216 & 0,034906585 & 0,628318531 & 0,020513409 & 0,028234286 & 0,999390827 & joint \\
\hline surface & 45 & 295 & 0,785398163 & 2,00712864 & 0,640856382 & $-0,298836239$ & 0,707106781 & joint \\
\hline surface & 59 & 238 & 0,541052068 & 1,012290966 & 0,436777059 & 0,272928598 & 0,857167301 & joint \\
\hline surface & 17 & 266 & 1,274090354 & 1,500983157 & 0,953975246 & 0,066708448 & 0,292371705 & joint \\
\hline surface & 46 & 274 & 0,767944871 & 1,640609497 & 0,692966218 & $-0,048456918$ & 0,7193398 & joint \\
\hline surface & 48 & 288 & 0,733038286 & 1,884955592 & 0,636381023 & $-0,206772729$ & 0,743144825 & joint \\
\hline surface & 47 & 220 & 0,750491578 & 0,698131701 & 0,438380096 & 0,522441054 & 0,731353702 & joint \\
\hline surface & 58 & 83 & 0,558505361 & $-1,692969374$ & $-0,525969326$ & $-0,064580913$ & 0,848048096 & joint \\
\hline surface & 89 & 218 & 0,017453293 & 0,663225116 & 0,010744774 & 0,013752684 & 0,999847695 & joint \\
\hline surface & 83 & 38 & 0,122173048 & $-2,478367538$ & $-0,07503026$ & $-0,096034353$ & 0,992546152 & joint \\
\hline surface & 57 & 249 & 0,575958653 & 1,204277184 & 0,508464343 & 0,195181174 & 0,838670568 & joint \\
\hline surface & 59 & 239 & 0,541052068 & 1,029744259 & 0,441473796 & 0,265264219 & 0,857167301 & joint \\
\hline surface & 72 & 222 & 0,314159265 & 0,733038286 & 0,206772729 & 0,22964438 & 0,951056516 & joint \\
\hline surface & 68 & 75 & 0,383972435 & $-1,832595715$ & $-0,361842183$ & $-0,096955321$ & 0,927183855 & joint \\
\hline surface & 87 & 53 & 0,052359878 & $-2,21656815$ & $-0,041797353$ & $-0,031496565$ & 0,998629535 & joint \\
\hline surface & 68 & 221 & 0,383972435 & 0,715584993 & 0,245764038 & 0,282719185 & 0,927183855 & joint \\
\hline surface & 66 & 221 & 0,41887902 & 0,715584993 & 0,266843247 & 0,306968041 & 0,913545458 & joint \\
\hline surface & 73 & 228 & 0,296705973 & 0,837758041 & 0,217274519 & 0,195634856 & 0,956304756 & joint \\
\hline surface & 49 & 205 & 0,715584993 & 0,436332313 & 0,277262526 & 0,594591407 & 0,75470958 & joint \\
\hline surface & 88 & 47 & 0,034906585 & $-2,321287905$ & $-0,025523876$ & $-0,0238014$ & 0,999390827 & joint \\
\hline surface & 68 & 90 & 0,383972435 & $-1,570796327$ & $-0,374606593$ & $2,29474 \mathrm{E}-17$ & 0,927183855 & joint \\
\hline
\end{tabular}




\begin{tabular}{|c|c|c|c|c|c|c|c|c|}
\hline \multirow{2}{*}{ depth (meter) } & \multicolumn{2}{|c|}{ measures } & \multirow{2}{*}{$\theta$} & \multirow{2}{*}{$\phi$} & \multicolumn{3}{|c|}{ cosine director } & \multirow{2}{*}{$\begin{array}{l}\text { geological } \\
\text { structure }\end{array}$} \\
\hline & $\operatorname{dip}$ & dipdirection & & & $\mathbf{x}$ & $\mathbf{y}$ & $\mathbf{z}$ & \\
\hline surface & 62 & 245 & 0,488692191 & 1,134464014 & 0,425485733 & 0,198407256 & 0,882947593 & joint \\
\hline surface & 62 & 242 & 0,488692191 & 1,082104136 & 0,414518786 & 0,220403548 & 0,882947593 & joint \\
\hline surface & 48 & 298 & 0,733038286 & 2,059488517 & 0,590807258 & $-0,314137791$ & 0,743144825 & joint \\
\hline surface & 47 & 291 & 0,750491578 & 1,93731547 & 0,63670032 & $-0,244406354$ & 0,731353702 & joint \\
\hline surface & 56 & 241 & 0,593411946 & 1,064650844 & 0,489081133 & 0,271102099 & 0,829037573 & joint \\
\hline surface & 89 & 216 & 0,017453293 & 0,628318531 & 0,010258267 & 0,014119293 & 0,999847695 & joint \\
\hline surface & 68 & 232 & 0,383972435 & 0,907571211 & 0,295194024 & 0,230630848 & 0,927183855 & joint \\
\hline surface & 61 & 228 & 0,506145483 & 0,837758041 & 0,360283761 & 0,324400955 & 0,874619707 & joint \\
\hline surface & 55 & 280 & 0,610865238 & 1,745329252 & 0,564862521 & $-0,099600503$ & 0,819152044 & joint \\
\hline surface & 88 & 167 & 0,034906585 & $-0,226892803$ & $-0,007850679$ & 0,034005025 & 0,999390827 & joint \\
\hline surface & 58 & 8 & 0,558505361 & $-3,001966313$ & $-0,073750507$ & $-0,524762126$ & 0,848048096 & joint \\
\hline surface & 50 & 266 & 0,698131701 & 1,500983157 & 0,641221811 & 0,044838597 & 0,7660444443 & joint \\
\hline surface & 83 & 227 & 0,122173048 & 0,820304748 & 0,089129595 & 0,083114692 & 0,992546152 & joint \\
\hline surface & 87 & 231 & 0,052359878 & 0,890117919 & 0,040672677 & 0,032936084 & 0,998629535 & joint \\
\hline surface & 86 & 235 & 0,06981317 & 0,959931089 & 0,057141158 & 0,04001067 & 0,99756405 & joint \\
\hline surface & 88 & 224 & 0,034906585 & 0,767944871 & 0,024243228 & 0,025104597 & 0,999390827 & joint \\
\hline surface & 61 & 50 & 0,506145483 & $-2,268928028$ & $-0,371385716$ & $-0,311629617$ & 0,874619707 & joint \\
\hline surface & 39 & 112 & 0,890117919 & $-1,186823891$ & $-0,720557188$ & 0,291124001 & 0,629320391 & joint \\
\hline surface & 56 & 286 & 0,593411946 & 1,850049007 & 0,537530719 & $-0,154134453$ & 0,829037573 & joint \\
\hline surface & 55 & 276 & 0,610865238 & 1,675516082 & 0,570434325 & $-0,059955063$ & 0,819152044 & joint \\
\hline surface & 86 & 11 & 0,06981317 & $-2,949606436$ & $-0,013310163$ & $-0,068474851$ & 0,99756405 & joint \\
\hline surface & 53 & 72 & 0,645771823 & $-1,884955592$ & $-0,572360099$ & $-0,18597107$ & 0,79863551 & joint \\
\hline surface & 88 & 232 & 0,034906585 & 0,907571211 & 0,027501179 & 0,021486276 & 0,999390827 & joint \\
\hline surface & 89 & 237 & 0,017453293 & 0,994837674 & 0,01463682 & 0,009505262 & 0,999847695 & joint \\
\hline surface & 62 & 218 & 0,488692191 & 0,663225116 & 0,289035555 & 0,36994864 & 0,882947593 & joint \\
\hline surface & 89 & 76 & 0,017453293 & $-1,815142422$ & $-0,016933995$ & $-0,004222119$ & 0,999847695 & joint \\
\hline surface & 84 & 60 & 0,104719755 & $-2,094395102$ & $-0,090524305$ & $-0,052264232$ & 0,994521895 & joint \\
\hline surface & 86 & 37 & 0,06981317 & $-2,49582083$ & $-0,041980494$ & $-0,055709997$ & 0,99756405 & joint \\
\hline surface & 87 & 230 & 0,052359878 & 0,872664626 & 0,040091668 & 0,033640904 & 0,998629535 & joint \\
\hline surface & 74 & 278 & 0,27925268 & 1,710422667 & 0,272954872 & $-0,038361306$ & 0,961261696 & joint \\
\hline surface & 54 & 284 & 0,628318531 & 1,815142422 & 0,570325518 & $-0,142198122$ & 0,809016994 & joint \\
\hline surface & 88 & 47 & 0,034906585 & $-2,321287905$ & $-0,025523876$ & $-0,0238014$ & 0,999390827 & joint \\
\hline surface & 61 & 285 & 0,506145483 & 1,832595715 & 0,468290133 & $-0,125477963$ & 0,874619707 & joint \\
\hline surface & 87 & 222 & 0,052359878 & 0,733038286 & 0,03501959 & 0,038893195 & 0,998629535 & joint \\
\hline surface & 56 & 271 & 0,593411946 & 1,588249619 & 0,559107736 & $-0,009759262$ & 0,829037573 & joint \\
\hline surface & 89 & 210 & 0,017453293 & 0,523598776 & 0,008726203 & 0,015114227 & 0,999847695 & joint \\
\hline surface & 89 & 241 & 0,017453293 & 1,064650844 & 0,015264219 & 0,008461095 & 0,999847695 & joint \\
\hline surface & 78 & 195 & 0,20943951 & 0,261799388 & 0,053811505 & 0,200827272 & 0,978147601 & joint \\
\hline surface & 89 & 230 & 0,017453293 & 0,872664626 & 0,013369319 & 0,011218191 & 0,999847695 & joint \\
\hline surface & 84 & 240 & 0,104719755 & 1,047197551 & 0,090524305 & 0,052264232 & 0,994521895 & joint \\
\hline surface & 57 & 273 & 0,575958653 & 1,623156204 & 0,543892626 & $-0,028504205$ & 0,838670568 & joint \\
\hline surface & 50 & 270 & 0,698131701 & 1,570796327 & 0,64278761 & $3,93755 \mathrm{E}-17$ & 0,766044443 & joint \\
\hline surface & 73 & 61 & 0,296705973 & $-2,07694181$ & $-0,255714055$ & $-0,141744615$ & 0,956304756 & joint \\
\hline surface & 62 & 279 & 0,488692191 & 1,727875959 & 0,463691589 & $-0,073441533$ & 0,882947593 & joint \\
\hline surface & 80 & 33 & 0,174532925 & $-2,565634$ & $-0,094575576$ & $-0,145633616$ & 0,984807753 & joint \\
\hline surface & 67 & 75 & 0,401425728 & $-1,832595715$ & $-0,377417288$ & $-0,101128658$ & 0,920504853 & joint \\
\hline surface & 71 & 310 & 0,331612558 & 2,268928028 & 0,249399676 & $-0,209271176$ & 0,945518576 & joint \\
\hline surface & 59 & 68 & 0,541052068 & $-1,954768762$ & $-0,477534988$ & $-0,192936659$ & 0,857167301 & joint \\
\hline surface & 33 & 298 & 0,994837674 & 2,059488517 & 0,740502159 & $-0,393731982$ & 0,544639035 & joint \\
\hline surface & 60 & 103 & 0,523598776 & $-1,343903524$ & $-0,487185032$ & 0,112475527 & 0,866025404 & joint \\
\hline surface & 65 & 221 & 0,436332313 & 0,715584993 & 0,277262526 & 0,318954051 & 0,906307787 & joint \\
\hline surface & 58 & 98 & 0,558505361 & $-1,431169987$ & $-0,524762126$ & 0,073750507 & 0,848048096 & joint \\
\hline surface & 41 & 284 & 0,855211333 & 1,815142422 & 0,73229148 & $-0,182580772$ & 0,656059029 & joint \\
\hline surface & 70 & 36 & 0,34906585 & $-2,513274123$ & $-0,201034396$ & $-0,276700108$ & 0,939692621 & joint \\
\hline surface & 80 & 38 & 0,174532925 & $-2,478367538$ & $-0,106908493$ & $-0,136836631$ & 0,984807753 & joint \\
\hline surface & 86 & 233 & 0,06981317 & 0,925024504 & 0,055709997 & 0,041980494 & 0,99756405 & joint \\
\hline surface & 62 & 186 & 0,488692191 & 0,104719755 & 0,049073141 & 0,4668999748 & 0,882947593 & joint \\
\hline
\end{tabular}




\begin{tabular}{|c|c|c|c|c|c|c|c|c|}
\hline \multirow{2}{*}{ depth (meter) } & \multicolumn{2}{|c|}{ measures } & \multirow{2}{*}{$\theta$} & \multirow{2}{*}{$\phi$} & \multicolumn{3}{|c|}{ cosine director } & \multirow{2}{*}{$\begin{array}{l}\text { geological } \\
\text { structure }\end{array}$} \\
\hline & $\operatorname{dip}$ & dipdirection & & & $\mathbf{x}$ & $\mathbf{y}$ & $\mathbf{z}$ & \\
\hline surface & 89 & 190 & 0,017453293 & 0,174532925 & 0,003030579 & 0,017187265 & 0,999847695 & joint \\
\hline surface & 89 & 35 & 0,017453293 & $-2,530727415$ & $-0,010010289$ & $-0,014296174$ & 0,999847695 & joint \\
\hline surface & 52 & 243 & 0,663225116 & 1,099557429 & 0,548558391 & 0,279504461 & 0,788010754 & joint \\
\hline surface & 45 & 255 & 0,785398163 & 1,308996939 & 0,683012702 & 0,183012702 & 0,707106781 & joint \\
\hline surface & 78 & 60 & 0,20943951 & $-2,094395102$ & $-0,180056806$ & $-0,103955845$ & 0,978147601 & joint \\
\hline surface & 89 & 33 & 0,017453293 & $-2,565634$ & $-0,009505262$ & $-0,01463682$ & 0,999847695 & joint \\
\hline surface & 68 & 58 & 0,383972435 & $-2,129301687$ & $-0,317684408$ & $-0,19851125$ & 0,927183855 & joint \\
\hline surface & 68 & 58 & 0,383972435 & $-2,129301687$ & $-0,317684408$ & $-0,19851125$ & 0,927183855 & joint \\
\hline surface & 73 & 63 & 0,296705973 & $-2,042035225$ & $-0,260505096$ & $-0,132733976$ & 0,956304756 & joint \\
\hline surface & 72 & 59 & 0,314159265 & $-2,111848395$ & $-0,264879263$ & $-0,159155518$ & 0,951056516 & joint \\
\hline surface & 70 & 344 & 0,34906585 & 2,862339973 & 0,094273528 & $-0,328770863$ & 0,939692621 & joint \\
\hline surface & 77 & 53 & 0,226892803 & $-2,21656815$ & $-0,1796539$ & $-0,135378924$ & 0,974370065 & joint \\
\hline surface & 59 & 271 & 0,541052068 & 1,588249619 & 0,514959632 & $-0,008988654$ & 0,857167301 & joint \\
\hline surface & 60 & 53 & 0,523598776 & $-2,21656815$ & $-0,399317755$ & $-0,300907512$ & 0,866025404 & joint \\
\hline surface & 30 & 277 & 1,047197551 & 1,692969374 & 0,859570182 & $-0,105541947$ & 0,5 & joint \\
\hline surface & 89 & 355 & 0,017453293 & 3,054326191 & 0,001521077 & $-0,017385995$ & 0,999847695 & joint \\
\hline surface & 87 & 30 & 0,052359878 & $-2,617993878$ & $-0,026167978$ & $-0,045324268$ & 0,998629535 & joint \\
\hline surface & 80 & 322 & 0,174532925 & 2,478367538 & 0,106908493 & $-0,136836631$ & 0,984807753 & joint \\
\hline surface & 89 & 40 & 0,017453293 & $-2,443460953$ & $-0,011218191$ & $-0,013369319$ & 0,999847695 & joint \\
\hline surface & 87 & 338 & 0,052359878 & 2,757620218 & 0,019605394 & $-0,048525054$ & 0,998629535 & joint \\
\hline surface & 80 & 333 & 0,174532925 & 2,670353756 & 0,078834623 & $-0,154721659$ & 0,984807753 & joint \\
\hline surface & 66 & 88 & 0,41887902 & $-1,605702912$ & $-0,40648887$ & $-0,014194904$ & 0,913545458 & joint \\
\hline surface & 78 & 343 & 0,20943951 & 2,844886681 & 0,060787495 & $-0,198826939$ & 0,978147601 & joint \\
\hline surface & 18 & 45 & 1,256637061 & $-2,35619449$ & $-0,672498512$ & $-0,672498512$ & 0,309016994 & joint \\
\hline surface & 41 & 274 & 0,855211333 & 1,640609497 & 0,752871146 & $-0,052645879$ & 0,656059029 & joint \\
\hline surface & 72 & 55 & 0,314159265 & $-2,181661565$ & $-0,253131903$ & $-0,177244866$ & 0,951056516 & joint \\
\hline surface & 67 & 54 & 0,401425728 & $-2,199114858$ & $-0,316108123$ & $-0,229665995$ & 0,920504853 & joint \\
\hline surface & 70 & 212 & 0,34906585 & 0,558505361 & 0,181243063 & 0,290049531 & 0,939692621 & joint \\
\hline surface & 85 & 211 & 0,087266463 & 0,541052068 & 0,044888526 & 0,074707053 & 0,996194698 & joint \\
\hline surface & 38 & 280 & 0,907571211 & 1,745329252 & 0,7760391 & $-0,136836631$ & 0,615661475 & joint \\
\hline surface & 71 & 60 & 0,331612558 & $-2,094395102$ & $-0,281950292$ & $-0,162784077$ & 0,945518576 & joint \\
\hline surface & 88 & 211 & 0,034906585 & 0,541052068 & 0,01797457 & 0,029914707 & 0,999390827 & joint \\
\hline surface & 83 & 215 & 0,122173048 & 0,610865238 & 0,069901384 & 0,099829522 & 0,992546152 & joint \\
\hline surface & 63 & 273 & 0,471238898 & 1,623156204 & 0,453368322 & $-0,023760027$ & 0,891006524 & joint \\
\hline surface & 60 & 33 & 0,523598776 & $-2,565634$ & $-0,272319518$ & $-0,419335284$ & 0,866025404 & joint \\
\hline surface & 40 & 278 & 0,872664626 & 1,710422667 & 0,758589351 & $-0,106612781$ & 0,64278761 & joint \\
\hline surface & 26 & 270 & 1,117010721 & 1,570796327 & 0,898794046 & $5,50578 \mathrm{E}-17$ & 0,438371147 & joint \\
\hline surface & 70 & 53 & 0,34906585 & $-2,21656815$ & $-0,273149432$ & $-0,20583286$ & 0,939692621 & joint \\
\hline surface & 82 & 218 & 0,13962634 & 0,663225116 & 0,085683517 & 0,1096699 & 0,990268069 & joint \\
\hline surface & 45 & 244 & 0,785398163 & 1,117010721 & 0,6355433365 & 0,309975211 & 0,707106781 & joint \\
\hline surface & 41 & 258 & 0,855211333 & 1,361356817 & 0,738217365 & 0,156912945 & 0,656059029 & joint \\
\hline surface & 72 & 55 & 0,314159265 & $-2,181661565$ & $-0,253131903$ & $-0,177244866$ & 0,951056516 & joint \\
\hline surface & 67 & 52 & 0,401425728 & $-2,234021443$ & $-0,307900331$ & $-0,240558103$ & 0,920504853 & joint \\
\hline surface & 53 & 56 & 0,645771823 & $-2,164208272$ & $-0,498927266$ & $-0,33653069$ & 0,79863551 & joint \\
\hline surface & 83 & 285 & 0,122173048 & 1,832595715 & 0,117716746 & $-0,031542107$ & 0,992546152 & joint \\
\hline surface & 67 & 58 & 0,401425728 & $-2,129301687$ & $-0,33135879$ & $-0,207055952$ & 0,920504853 & joint \\
\hline surface & 38 & 265 & 0,907571211 & 1,483529864 & 0,785012135 & 0,068679663 & 0,615661475 & joint \\
\hline surface & 63 & 41 & 0,471238898 & $-2,42600766$ & $-0,297844566$ & $-0,342630979$ & 0,891006524 & joint \\
\hline surface & 82 & 30 & 0,13962634 & $-2,617993878$ & $-0,06958655$ & $-0,120527441$ & 0,990268069 & joint \\
\hline surface & 89 & 309 & 0,017453293 & 2,251474735 & 0,013563067 & $-0,010983155$ & 0,999847695 & joint \\
\hline surface & 80 & 33 & 0,174532925 & $-2,565634$ & $-0,094575576$ & $-0,145633616$ & 0,984807753 & joint \\
\hline surface & 88 & 353 & 0,034906585 & 3,019419606 & 0,004253179 & $-0,034639361$ & 0,999390827 & joint \\
\hline surface & 38 & 273 & 0,907571211 & 1,623156204 & 0,786930812 & $-0,041241296$ & 0,615661475 & joint \\
\hline surface & 62 & 31 & 0,488692191 & $-2,600540585$ & $-0,24179573$ & $-0,402415672$ & 0,882947593 & joint \\
\hline surface & 60 & 53 & 0,523598776 & $-2,21656815$ & $-0,399317755$ & $-0,300907512$ & 0,866025404 & joint \\
\hline surface & 78 & 211 & 0,20943951 & 0,541052068 & 0,107082437 & 0,178215103 & 0,978147601 & joint \\
\hline surface & 53 & 38 & 0,645771823 & $-2,478367538$ & $-0,370514325$ & $-0,47423671$ & 0,79863551 & joint \\
\hline
\end{tabular}




\begin{tabular}{|c|c|c|c|c|c|c|c|c|}
\hline \multirow{2}{*}{ depth (meter) } & \multicolumn{2}{|c|}{ measures } & \multirow{2}{*}{$\boldsymbol{\theta}$} & \multirow{2}{*}{$\phi$} & \multicolumn{3}{|c|}{ cosine director } & \multirow{2}{*}{$\begin{array}{l}\text { geological } \\
\text { structure }\end{array}$} \\
\hline & dip & dipdirection & & & $\mathbf{x}$ & \begin{tabular}{|c|}
$\mathbf{y}$ \\
\end{tabular} & $\mathbf{z}$ & \\
\hline surface & 85 & 218 & 0,087266463 & 0,663225116 & 0,053658433 & 0,068679663 & 0,996194698 & joint \\
\hline surface & 85 & 208 & 0,087266463 & 0,488692191 & 0,040917143 & 0,076953953 & 0,996194698 & joint \\
\hline surface & 88 & 222 & 0,034906585 & 0,733038286 & 0,023352321 & 0,02593538 & 0,999390827 & joint \\
\hline surface & 73 & 54 & 0,296705973 & $-2,199114858$ & $-0,236533678$ & $-0,171851776$ & 0,956304756 & joint \\
\hline surface & 66 & 52 & 0,41887902 & $-2,234021443$ & $-0,320512849$ & $-0,250412082$ & 0,913545458 & joint \\
\hline surface & 48 & 247 & 0,733038286 & 1,169370599 & 0,615937971 & 0,261450157 & 0,743144825 & joint \\
\hline surface & 75 & 46 & 0,261799388 & $-2,338741198$ & $-0,18617884$ & $-0,179790816$ & 0,965925826 & joint \\
\hline surface & 84 & 32 & 0,104719755 & $-2,583087293$ & $-0,055391646$ & $-0,088645164$ & 0,994521895 & joint \\
\hline surface & 37 & 263 & 0,925024504 & 1,448623279 & 0,792682602 & 0,097329185 & 0,601815023 & joint \\
\hline surface & 63 & 58 & 0,471238898 & $-2,129301687$ & $-0,385005779$ & $-0,240578312$ & 0,891006524 & joint \\
\hline surface & 82 & 158 & 0,13962634 & $-0,383972435$ & $-0,052135161$ & 0,129039052 & 0,990268069 & joint \\
\hline surface & 65 & 38 & 0,436332313 & $-2,478367538$ & $-0,260189783$ & $-0,333027735$ & 0,906307787 & joint \\
\hline surface & 88 & 221 & 0,034906585 & 0,715584993 & 0,02289613 & 0,026338985 & 0,999390827 & joint \\
\hline surface & 85 & 214 & 0,087266463 & 0,593411946 & 0,048736873 & 0,072255385 & 0,996194698 & joint \\
\hline surface & 73 & 54 & 0,296705973 & $-2,199114858$ & $-0,236533678$ & $-0,171851776$ & 0,956304756 & joint \\
\hline surface & 72 & 210 & 0,314159265 & 0,523598776 & 0,154508497 & 0,267616567 & 0,951056516 & joint \\
\hline surface & 40 & 282 & 0,872664626 & 1,780235837 & 0,749304534 & $-0,159269595$ & 0,64278761 & joint \\
\hline surface & 68 & 167 & 0,383972435 & $-0,226892803$ & $-0,084268148$ & 0,365005451 & 0,927183855 & joint \\
\hline surface & 74 & 55 & 0,27925268 & $-2,181661565$ & $-0,225788903$ & $-0,158099092$ & 0,961261696 & joint \\
\hline surface & 80 & 38 & 0,174532925 & $-2,478367538$ & $-0,106908493$ & $-0,136836631$ & 0,984807753 & joint \\
\hline surface & 88 & 23 & 0,034906585 & $-2,740166926$ & $-0,01363632$ & $-0,032125156$ & 0,999390827 & joint \\
\hline surface & 83 & 28 & 0,122173048 & $-2,652900463$ & $-0,057214191$ & $-0,107604243$ & 0,992546152 & joint \\
\hline surface & 79 & 171 & 0,191986218 & $-0,157079633$ & $-0,029849103$ & 0,18845982 & 0,981627183 & joint \\
\hline surface & 78 & 25 & 0,20943951 & $-2,705260341$ & $-0,087867277$ & $-0,188431984$ & 0,978147601 & joint \\
\hline surface & 79 & 23 & 0,191986218 & $-2,740166926$ & $-0,074555014$ & $-0,175640606$ & 0,981627183 & joint \\
\hline surface & 78 & 38 & 0,20943951 & $-2,478367538$ & $-0,128003218$ & $-0,163836648$ & 0,978147601 & joint \\
\hline surface & 72 & 11 & 0,314159265 & $-2,949606436$ & $-0,058963222$ & $-0,303339482$ & 0,951056516 & joint \\
\hline surface & 56 & 13 & 0,593411946 & $-2,914699851$ & $-0,125791033$ & $-0,544860826$ & 0,829037573 & joint \\
\hline surface & 56 & 13 & 0,593411946 & $-2,914699851$ & $-0,125791033$ & $-0,544860826$ & 0,829037573 & joint \\
\hline surface & 65 & 58 & 0,436332313 & $-2,129301687$ & $-0,358400612$ & $-0,223953558$ & 0,906307787 & joint \\
\hline surface & 76 & 200 & 0,244346095 & 0,34906585 & 0,082742161 & 0,22733222 & 0,970295726 & joint \\
\hline surface & 81 & 213 & 0,157079633 & 0,575958653 & 0,085200316 & 0,131196982 & 0,987688341 & joint \\
\hline surface & 85 & 216 & 0,087266463 & 0,628318531 & 0,05122886 & 0,070510477 & 0,996194698 & joint \\
\hline surface & 88 & 247 & 0,034906585 & 1,169370599 & 0,032125156 & 0,01363632 & 0,999390827 & joint \\
\hline surface & 79 & 278 & 0,191986218 & 1,710422667 & 0,188952055 & $-0,02655548$ & 0,981627183 & joint \\
\hline surface & 37 & 118 & 0,925024504 & $-1,082104136$ & $-0,705153301$ & 0,374936661 & 0,601815023 & joint \\
\hline surface & 81 & 218 & 0,157079633 & 0,663225116 & 0,096310674 & 0,123272041 & 0,987688341 & joint \\
\hline surface & 87 & 210 & 0,052359878 & 0,523598776 & 0,026167978 & 0,045324268 & 0,998629535 & joint \\
\hline surface & 87 & 208 & 0,052359878 & 0,488692191 & 0,024570243 & 0,046209907 & 0,998629535 & joint \\
\hline surface & 71 & 101 & 0,331612558 & $-1,378810109$ & $-0,31958655$ & 0,062121332 & 0,945518576 & joint \\
\hline surface & 89 & 28 & 0,017453293 & $-2,652900463$ & $-0,008193409$ & $-0,01540956$ & 0,999847695 & joint \\
\hline surface & 82 & 210 & 0,13962634 & 0,523598776 & 0,06958655 & 0,120527441 & 0,990268069 & joint \\
\hline surface & 44 & 280 & 0,802851456 & 1,745329252 & 0,708411412 & $-0,124912045$ & 0,69465837 & joint \\
\hline surface & 85 & 223 & 0,087266463 & 0,750491578 & 0,059440074 & 0,063741675 & 0,996194698 & joint \\
\hline surface & 80 & 208 & 0,174532925 & 0,488692191 & 0,081522881 & 0,15332224 & 0,984807753 & joint \\
\hline surface & 85 & 18 & 0,087266463 & $-2,827433388$ & $-0,026932606$ & $-0,082890037$ & 0,996194698 & joint \\
\hline surface & 87 & 37 & 0,052359878 & $-2,49582083$ & $-0,031496565$ & $-0,041797353$ & 0,998629535 & joint \\
\hline surface & 87 & 246 & 0,052359878 & 1,151917306 & 0,047811275 & 0,021286951 & 0,998629535 & joint \\
\hline surface & 81 & 59 & 0,157079633 & $-2,111848395$ & $-0,134090508$ & $-0,080569706$ & 0,987688341 & joint \\
\hline surface & 80 & 53 & 0,174532925 & $-2,21656815$ & $-0,138681601$ & $-0,104504082$ & 0,984807753 & joint \\
\hline surface & 85 & 171 & 0,087266463 & $-0,157079633$ & $-0,013634162$ & 0,086082711 & 0,996194698 & joint \\
\hline surface & 70 & 330 & 0,34906585 & 2,617993878 & 0,171010072 & $-0,296198133$ & 0,939692621 & joint \\
\hline surface & 89 & 333 & 0,017453293 & 2,670353756 & 0,007923227 & $-0,015550208$ & 0,999847695 & joint \\
\hline surface & 33 & 272 & 0,994837674 & 1,605702912 & 0,838159672 & $-0,029269181$ & 0,544639035 & joint \\
\hline surface & 75 & 207 & 0,261799388 & 0,471238898 & 0,117501388 & 0,230609458 & 0,965925826 & joint \\
\hline surface & 51 & 156 & 0,680678408 & $-0,41887902$ & $-0,255967663$ & 0,574912785 & 0,777145961 & joint \\
\hline surface & 88 & 319 & 0,034906585 & 2,42600766 & 0,02289613 & $-0,026338985$ & 0,999390827 & joint \\
\hline
\end{tabular}




\begin{tabular}{|c|c|c|c|c|c|c|c|c|}
\hline \multirow{2}{*}{ depth (meter) } & \multicolumn{2}{|c|}{ measures } & \multirow{2}{*}{$\boldsymbol{\theta}$} & \multirow{2}{*}{$\phi$} & \multicolumn{3}{|c|}{ cosine director } & \multirow{2}{*}{$\begin{array}{l}\text { geological } \\
\text { structure }\end{array}$} \\
\hline & dip & dipdirection & & & $\mathbf{x}$ & $\mathbf{y}$ & $\mathbf{z}$ & \\
\hline surface & 65 & \begin{tabular}{|l|}
158 \\
\end{tabular} & 0,436332313 & $-0,383972435$ & $-0,158315587$ & 0,391844829 & 0,906307787 & joint \\
\hline surface & 83 & 201 & 0,122173048 & 0,366519143 & 0,043674067 & 0,113774834 & 0,992546152 & joint \\
\hline surface & 46 & 50 & 0,767944871 & $-2,268928028$ & $-0,532139185$ & $-0,446517793$ & 0,7193398 & joint \\
\hline surface & 52 & 48 & 0,663225116 & $-2,303834613$ & $-0,45752564$ & $-0,411957936$ & 0,788010754 & joint \\
\hline surface & 65 & 35 & 0,436332313 & $-2,530727415$ & $-0,242403877$ & $-0,346188613$ & 0,906307787 & joint \\
\hline surface & 82 & 23 & 0,13962634 & $-2,740166926$ & $-0,054379263$ & $-0,128109515$ & 0,990268069 & joint \\
\hline surface & 80 & 25 & 0,174532925 & $-2,705260341$ & $-0,073386891$ & $-0,157378696$ & 0,984807753 & joint \\
\hline surface & 53 & 204 & 0,645771823 & 0,41887902 & 0,244780222 & 0,549785381 & 0,79863551 & joint \\
\hline surface & 66 & 352 & 0,41887902 & 3,001966313 & 0,0566068 & $-0,40277831$ & 0,913545458 & joint \\
\hline surface & 52 & 272 & 0,663225116 & 1,605702912 & 0,615286431 & $-0,021486276$ & 0,788010754 & joint \\
\hline surface & 89 & 223 & 0,017453293 & 0,750491578 & 0,011902513 & 0,012763882 & 0,999847695 & joint \\
\hline surface & 84 & 205 & 0,104719755 & 0,436332313 & 0,044175637 & 0,09473496 & 0,994521895 & joint \\
\hline surface & 87 & 240 & 0,052359878 & 1,047197551 & 0,045324268 & 0,026167978 & 0,998629535 & joint \\
\hline surface & 77 & 47 & 0,226892803 & $-2,321287905$ & $-0,164518786$ & $-0,15341625$ & 0,974370065 & joint \\
\hline surface & 87 & 43 & 0,052359878 & $-2,391101075$ & $-0,035693036$ & $-0,038276095$ & 0,998629535 & joint \\
\hline surface & 48 & 276 & 0,733038286 & 1,675516082 & 0,665465039 & $-0,069943194$ & 0,743144825 & joint \\
\hline surface & 81 & 27 & 0,157079633 & $-2,670353756$ & $-0,071019761$ & $-0,139384129$ & 0,987688341 & joint \\
\hline surface & 77 & 210 & 0,226892803 & 0,523598776 & 0,112475527 & 0,194813328 & 0,974370065 & joint \\
\hline surface & 54 & 274 & 0,628318531 & 1,640609497 & 0,586353437 & $-0,041001827$ & 0,809016994 & joint \\
\hline surface & 56 & 279 & 0,593411946 & 1,727875959 & 0,552308311 & $-0,087477043$ & 0,829037573 & joint \\
\hline surface & 65 & 62 & 0,436332313 & $-2,059488517$ & $-0,373149777$ & $-0,198407256$ & 0,906307787 & joint \\
\hline surface & 47 & 239 & 0,750491578 & 1,029744259 & 0,5845866693 & 0,351255122 & 0,731353702 & joint \\
\hline surface & 89 & 92 & 0,017453293 & $-1,535889742$ & $-0,017441775$ & 0,00060908 & 0,999847695 & joint \\
\hline surface & 89 & 91 & 0,017453293 & $-1,553343034$ & $-0,017449748$ & 0,000304586 & 0,999847695 & joint \\
\hline surface & 89 & 92 & 0,017453293 & $-1,535889742$ & $-0,017441775$ & 0,00060908 & 0,999847695 & joint \\
\hline surface & 89 & 118 & 0,017453293 & $-1,082104136$ & $-0,01540956$ & 0,008193409 & 0,999847695 & joint \\
\hline surface & 89 & 120 & 0,017453293 & $-1,047197551$ & $-0,015114227$ & 0,008726203 & 0,999847695 & joint \\
\hline surface & 88 & 209 & 0,034906585 & 0,506145483 & 0,016919612 & 0,030523788 & 0,999390827 & joint \\
\hline surface & 88 & 210 & 0,034906585 & 0,523598776 & 0,017449748 & 0,030223851 & 0,999390827 & joint \\
\hline surface & 89 & 210 & 0,017453293 & 0,523598776 & 0,008726203 & 0,015114227 & 0,999847695 & joint \\
\hline surface & 89 & 217 & 0,017453293 & 0,645771823 & 0,01050312 & 0,013938112 & 0,999847695 & joint \\
\hline surface & 89 & 244 & 0,017453293 & 1,117010721 & 0,015686119 & 0,007650631 & 0,999847695 & joint \\
\hline surface & 64 & 255 & 0,453785606 & 1,308996939 & 0,423434012 & 0,113458802 & 0,898794046 & joint \\
\hline surface & 88 & 300 & 0,034906585 & 2,094395102 & 0,030223851 & $-0,017449748$ & 0,999390827 & joint \\
\hline surface & 75 & 337 & 0,261799388 & 2,740166926 & 0,101128658 & $-0,238244187$ & 0,965925826 & joint \\
\hline surface & 89 & 10 & 0,017453293 & $-2,967059728$ & $-0,003030579$ & $-0,017187265$ & 0,999847695 & joint \\
\hline surface & 84 & 35 & 0,104719755 & $-2,530727415$ & $-0,059955063$ & $-0,085624704$ & 0,994521895 & joint \\
\hline surface & 76 & 65 & 0,244346095 & $-2,00712864$ & $-0,219255698$ & $-0,102240611$ & 0,970295726 & joint \\
\hline surface & 80 & 75 & 0,174532925 & $-1,832595715$ & $-0,167731259$ & $-0,044943456$ & 0,984807753 & joint \\
\hline
\end{tabular}




\section{Supplementary Material 2}

407

408

409

410

411

412

413

414

415

416

dados= read.csv("Dados_Totais_Prof.csv",sep=";")

dados_frat $=$ dados[dados\$estrutura=="fratura",]

dados_fol = dados[dados\$estrutura=="foliacao",]

dados_fratura = read.csv("fratura.csv",sep=";")

\#Usando lm (regressao multivariada)

$\mathrm{X}=$ dados $\_4 \mathrm{~d} \$ \mathrm{x}$

$\mathrm{Y}=$ dados_4d $\$ \mathrm{y}$

$\mathrm{Z}=$ dados $\_4 \mathrm{~d} \$ \mathrm{z}$

estr $=$ factor(dados_ $\_$d $\$$ estrutura $)$

prof $=($ as.numeric $($ dados_4d $\$$ profundidade $))$

fit. $\operatorname{lm}<-\operatorname{lm}(\operatorname{cbind}(\mathrm{x}, \mathrm{y}, \mathrm{z}) \sim$ as.numeric(profundidade)+estrutura+as.numeric(profundidade)*estrutura, data $=$ dados_4d)

summary(fit.lm, multivariate=TRUE)

\#diagnostico

plot(fit.lm\$fitted.values[,1],fit.lm\$residuals[,1],xlab = "Valor ajustado de X", ylab = "Resíduo")

plot(fit.lm\$fitted.values[,2],fit.lm\$residuals[,2],xlab = "Valor ajustado de y", ylab = "Resíduo")

plot(fit.lm\$fitted.values[,3],fit.lm\$residuals[,3],xlab = "Valor ajustado de z", ylab = "Resíduo")

car::qqPlot(as.numeric(fit.lm\$residuals[,1]),ylab = "Resíduo de x")

car::qqPlot(as.numeric(fit.lm\$residuals[,2]),ylab = "Resíduo de y")

car::qqPlot(as.numeric(fit.lm\$residuals[,3]),ylab = "Resíduo de z")

\#como deu interacao, vamos separar as estruturas:

dados_fol = dados[dados\$estrutura=="foliacao",]

scatter3D(dados_fol\$x,dados_fol\$y,dados_fol\$z,colvar=FALSE,col = "blue")

\#T1

estr $=$ factor(dados_ $\_$d $\$$ estrutura $)$

prof $=($ as.numeric $($ dados_ $4 \mathrm{~d} \$$ profundidade $))$

fit.lm_fol $=\operatorname{lm}(\operatorname{cbind}(\mathrm{x}, \mathrm{y}, \mathrm{z}) \sim$ profundidade, data $=$ dados_fol $)$

\#diagnostico 
plot(fit.lm_fol\$fitted.values[,1],fit.lm_fol\$residuals[,1],xlab = "Valor ajustado de X", ylab = "Resíduo",main

445 ="Numérico")

plot(fit.lm_fol\$fitted.values[,2],fit.lm_fol\$residuals[,2],xlab = "Valor ajustado de y", ylab = "Resíduo",main

447 ="Numérico")

448 plot(fit.lm_fol\$fitted.values[,3],fit.lm_fol\$residuals[,3],xlab = "Valor ajustado de z", ylab = "Resíduo",main 449 ="Numérico")

450 car::qqPlot(as.numeric(fit.lm_fol\$residuals[,1]), main ="Numérico")

451 car::qqPlot(as.numeric(fit.lm_fol\$residuals[,2]), main ="Numérico")

452 car::qqPlot(as.numeric(fit.lm_fol\$residuals[,3]), main ="Numérico")

455 \#usando funcao manova

$456 \quad \mathrm{X}=$ dados_4d $\$ \mathrm{X}$

$457 \quad \mathrm{Y}=$ dados_4d $\$ \mathrm{y}$

$458 \quad \mathrm{Z}=$ dados_4d $\$ \mathrm{z}$

459 aux_prof $=$ dados_4d\$aux

fit. $\operatorname{lm} X=\operatorname{lm}($ as.numeric $(\mathrm{X}) \sim$ factor(aux_prof)+factor(etsr)+factor(aux_prof)*factor(estr))

plot(as.numeric(X), fit.lmX\$residuals)

car::qqPlot(fit.lmX\$residuals)

fit.lmX $=$ lme(as.numeric(X) factor(aux_prof)+factor(etsr)+factor(aux_prof)*factor(estr))

\#diagnostico 
485 fit.manova <- Manova(fit.lm)

486 summary(fit.manova, multivariate=TRUE)

487 summary(fit2.manova)

488 summary(fit2.lm)

$\operatorname{par}(\mathrm{mfrow}=\mathrm{c}(1,1))$

plot(fit.lm\$fitted.values[,1],fit.lm\$residuals[,1], xlab = "Valor ajustado de x", ylab = "Resíduo")

$\operatorname{par}(\mathrm{mfrow}=\mathrm{c}(1,1))$

\section{Supplementary Material 3}

teto $=1$

$$
\text { teto }=\text { valor }
$$


525 piso_min $=$ function $($ valor $)\{$

526 piso $=-1$

527 if(piso $<-1)\{$

528 piso $=$ valor

$529\}$

530 valor

$531\}$

532

533 \#Gera regioes de confiança com default de $\mathrm{p}$-valor=5\% e m=1000000 pontos simulados e $\mathrm{k}$ graus de liberdade

534 mi_ic $=$ function $($ dados, quantil $=0.95, \mathrm{~m}=1000000, \mathrm{k})\{$

$535 \mathrm{mi}=\mathrm{NULL}$

$536 \quad \mathrm{xyz}=\operatorname{cbind}(\operatorname{dados} \$ \mathrm{x}, \operatorname{dados} \$ \mathrm{y}, \operatorname{dados} \$ \mathrm{z})$

$537 \mathrm{n}=$ length $(\operatorname{dados} \$ \mathrm{x})$

$538 \quad x y z \_b a r=\operatorname{rbind}(\operatorname{mean}(\operatorname{dados} \$ \mathrm{x}), \operatorname{mean}(\operatorname{dados} \$ y), \operatorname{mean}(\operatorname{dados} \$ z))$

539 var_xyz $=\operatorname{diag}(\operatorname{cov}(x y z))$

540 x_ic $=c\left(\right.$ piso_min $\left.\left(x y z \_b a r[1]-2 * v a r \_x y z[1]\right), t e t o \_m a x\left(x y z \_b a r[1]+2 * v a r \_x y z[1]\right)\right)$

541 y_ic $=c\left(\right.$ piso_min $\left(x y z \_b a r[2]-2 * v a r \_x y z[2]\right)$,teto_max $\left.\left(x y z \_b a r[2]+2 * v a r \_x y z[2]\right)\right)$

542 z_ic $=c\left(\right.$ piso_min $\left(x y z \_b a r[3]-2 * v a r \_x y z[3]\right)$, teto_max $\left.\left(x y z \_b a r[3]+2 * v a r \_x y z[3]\right)\right)$

543 x_tilde $=$ xyz[1,]-xyz_bar

544 for(i in $2: n)\{$

$545 \quad$ x_tilde $=\operatorname{cbind}\left(x \_t i l d e, x y z[i]-,x y z \_b a r\right)$

$546 \quad\}$

547 S0 $=$ x_tilde $\% * \% t\left(x \_t i l d e\right)$

548 So_inv $=\operatorname{solve}(\mathrm{S} 0)^{*} \mathrm{n}$

549 for $(\mathrm{j}$ in $1: \mathrm{m})\{$

550 mi_new $=\mathrm{c}\left(\operatorname{runif}\left(1, \min =x \_i c[1], \max =x \_i c[2]\right), \operatorname{runif}\left(1, \min =y \_i c[1], \max =y \_i c[2]\right), \operatorname{runif}\left(1, \min =z \_i c[1], \max =z \_i c[2]\right)\right)$

$551 \quad$ central $=\mathrm{t}\left(\mathrm{xyz} \_\right.$bar-mi_new $)$

$552 \mathrm{~A}=$ central\%*\%S0_inv\%*\%t(central)

$553 \quad$ if $(\mathrm{A}<\mathrm{k})\{$

554 mi $=$ cbind $\left(m i, m i \_n e w\right)$

555

\}

$556\}$

$557 \mathrm{mi}=$ as.data.frame $(\mathrm{mi})$

$558\}$

559 range_mi $=$ function $(\mathrm{mi})\{$ 
564 limits $=$ as.data.frame(limits)

\#elipsoide geral

\#\#calculo da constante ck

$\mathrm{n}=$ length(dados_sup $\$ \mathrm{x}$ )

$\mathrm{n} 2=$ length(dados_prof $\$ \mathrm{x})$

574 quantil $=0.95$

$575 \quad \mathrm{ck}=3 /(\mathrm{n}+\mathrm{n} 2-3) * \mathrm{qf}(\mathrm{quantil}, 3, \mathrm{n}+\mathrm{n} 2-3)$

576 \#\#simulacao dos pontos de superficie e profundidade

577 mi_sup $=$ mi_ic(dados_sup, $\mathrm{k}=\mathrm{ck})$

578 mi_prof $=$ mi_ic $($ dados_prof, $\mathrm{k}=\mathrm{ck})$

579 \#\#categorizacao dos pontos

580 mi_sup_cat $=$ rbind $($ mi_sup, 1$)$

581 mi_prof_cat $=$ rbind $($ mi_prof, 2$)$

582 mi_tot $=$ as.data.frame $\left(\mathrm{t}\left(\mathrm{cbind}\left(\mathrm{mi} \_s u p \_c a t, m i \_p r o f \_c a t\right)\right)\right)$

583 colnames(mi_tot) = c("x","y","z","sup_prof")

584 range_mi_mi_tot $=$ range_mi $\left(m i \_t o t\right)$

585 \#\#plot

586 plot3d(mi_tot\$x,mi_tot\$y,mi_tot\$z, xlim = range_mi_mi_tot $\$ \mathrm{x}, \mathrm{ylim}$ range_mi_mi_tot $\$ \mathbf{z}, \mathrm{col}=\mathrm{mi} \_$tot$\$$ sup_prof)

\#elipsoide para foliacao

\#\#calculo da constante ck

$\mathrm{n}=$ length(dados_fol_sup\$ $\mathrm{x}$ )

$\mathrm{n} 2=$ length (dados_fol_prof $\$ \mathrm{x})$

$\mathrm{ck}=3 /(\mathrm{n}+\mathrm{n} 2-3) * \mathrm{qf}(\mathrm{quantil}, 3, \mathrm{n}+\mathrm{n} 2-3)$

\#\#simulacao dos pontos de superficie e profundidade

mi_fol_sup $=$ mi_ic $($ dados_fol_sup, $\mathrm{k}=\mathrm{ck})$

mi_fol_prof $=$ mi_ic $($ dados_fol_prof, $\mathrm{k}=\mathrm{ck})$ 
mi_fol_prof_cat $=$ rbind $($ mi_fol_prof,2)

mi_fol_tot $=$ as.data.frame $\left(\mathrm{t}\left(\mathrm{cbind}\left(\mathrm{mi} \_f o l \_s u p \_c a t, m i \_f o l \_p r o f \_c a t\right)\right)\right)$

601 colnames(mi_fol_tot) = c("x","y","z","sup_prof")

602 range_mi_mi_tot $=$ range_mi $($ mi_fol_tot $)$

603 \#\#plot

604 plot3d(mi_fol_tot $\$ \mathrm{x}, \mathrm{mi} \_f o l \_t o t \$ y, m i \_f o l \_t o t \$ z, \quad x l i m=$ range_mi_mi_tot $\$ \mathrm{x}, \mathrm{ylim}=$ range_mi_mi_tot $\$ \mathrm{y}, \mathrm{zlim}=$ 605 range_mi_mi_tot\$z,col=mi_fol_tot\$sup_prof,xlab="x",ylab="y",zlab="z")

606

607

608 \#elipsoide para fratura

609 \#\#calculo da constante ck

$610 \mathrm{n}=$ length(dados_frat_sup $\$ \mathrm{x}$ )

$611 \mathrm{n} 2=$ length $($ dados_frat_prof $\$ \mathrm{x})$

$612 \mathrm{ck}=3 /(\mathrm{n}+\mathrm{n} 2-3) * \mathrm{qf}($ quantil,3,n+n2-3)

613 \#\#simulacao dos pontos de superficie e profundidade

614 mi_frat_sup $=$ mi_ic $($ dados_frat_sup, $\mathrm{k}=\mathrm{ck})$

615 mi_frat_prof $=$ mi_ic $($ dados_frat_prof, $\mathrm{k}=\mathrm{ck})$

616 \#\#categorizacao dos pontos

617 mi_frat_sup_cat $=$ rbind $($ mi_frat_sup, 1$)$

618 mi_frat_prof_cat $=$ rbind(mi_frat_prof,2)

619 mi_frat_tot $=$ as.data.frame $\left(t\left(\right.\right.$ cbind $\left.\left.\left(m i \_s u p \_c a t, m i \_p r o f \_c a t\right)\right)\right)$

620 range_mi_mi_tot $=$ range_mi $($ mi_frat_tot $)$

621 colnames(mi_frat_tot) = c("x","y","z","sup_prof") plot3d(mi_frat_tot\$x,mi_frat_tot\$y,mi_frat_tot $\$ \mathrm{z}, \mathrm{xlim}=$ range_mi_mi_tot $\$ \mathrm{x}, \mathrm{ylim}=$ range_mi_mi_tot\$y,zlim = range_mi_mi_tot\$z,col=mi_frat_tot\$sup_prof,xlab="x",ylab="y",zlab="z")

\section{Supplementary Material 4}

dados= read.csv("Dados_Totais_Prof.csv",sep=";")

dados_frat $=$ dados[dados\$estrutura=="fratura",]

dados_fol = dados[dados\$estrutura=="foliacao",]

631 dados_frat_prof $=$ dados_frat[dados_frat\$sup_prof==2,]

632 dados_fol_sup $=$ dados_fol[dados_fol\$sup_prof $==1$, ]

633 dados_fol_prof $=$ dados_fol[dados_fol\$sup_prof==2,]

634 dados_sup $=$ dados[dados\$sup_prof==1,]

635 dados_prof $=$ dados[dados\$sup_prof $==2$,] 
$639\}$

640

641 qhotelling $=$ function $(\mathrm{p}, \mathrm{v})\{$

$642 \mathrm{qf}(\mathrm{p}, 3, \mathrm{v}-3) *(\mathrm{v}-1) * 3 /(\mathrm{v}-3)$

$643\}$

644

645 \#Dá o quantil da T2 de Hotelling associada

646 quantil_T2 $=$ function(dados,quantil $)\{$

647 dados_s $=$ dados[dados $\$$ sup_prof $==1$,]

648 dados_p $=$ dados[dados\$sup_prof $==2$,

649 xyz_s $=$ cbind $\left(\right.$ dados_s $\left.\$ \mathrm{x}, \mathrm{dados} \_\mathrm{S} \$ \mathrm{y}, \mathrm{dados} \_\mathrm{s} \$ \mathrm{z}\right)$

650 xyz_p $=$ cbind (dados_p\$x,dados_p\$y,dados_p\$z)

$651 \mathrm{n} 1=$ length $\left(x_{y z} \_\mathrm{s}[, 1]\right)$

$652 \mathrm{n} 2=$ length $\left(x_{y z} \_\mathrm{p}[, 1]\right)$

653 xyz_bar_s $=\operatorname{cbind}\left(\operatorname{mean}\left(x y z \_s[, 1]\right), \operatorname{mean}\left(x y z \_s[, 2]\right)\right.$, mean $\left.\left(x y z \_s[, 3]\right)\right)$

654 xyz_bar_p = cbind $\left(\operatorname{mean}\left(x y z \_p[, 1]\right), \operatorname{mean}\left(x y z \_p[, 2]\right), \operatorname{mean}\left(x y z \_p[, 3]\right)\right)$

$655 \quad \mathrm{~S} 1=\operatorname{cov}\left(\mathrm{xyz}_{-} \mathrm{s}\right) / \mathrm{n} 1$

656 S2 $=\operatorname{cov}\left(x y z \_p\right) / n 2$

657 mid_fol $=\mathrm{S} 1+\mathrm{S} 2$

658 mid_fol_inv $=$ solve(mid_fol)

$659 \mathrm{v}=\left(\operatorname{tr}(\right.$ mid_fol\%*\%mid_fol $\left.)+(\operatorname{tr}(\text { mid_fol }))^{\wedge} 2\right) /\left(\left(\operatorname{tr}(\mathrm{S} 1 \% * \% \mathrm{~S} 1)+(\operatorname{tr}(\mathrm{S} 1))^{\wedge} 2\right) /(\mathrm{n} 1-1)\right)+\left(\left(\operatorname{tr}(\mathrm{S} 2 \% * \% \mathrm{~S} 2)+(\operatorname{tr}(\mathrm{S} 2))^{\wedge} 2\right) /(\mathrm{n} 2-\right.$ $66011)$

661 qhotelling(quantil,v)

$662\}$

663

664 quantil_T2(dados_fol,0.95)

665

666 \#construindo a estatística $\mathrm{T}^{\wedge} 2$ de Hotelling

n_fol_sup $=$ length $\left(x y z \_f o l \_s u p[, 1]\right)$

668

n_fol_prof $=$ length $\left(x y z \_f o l \_p r o f[, 1]\right)$

669

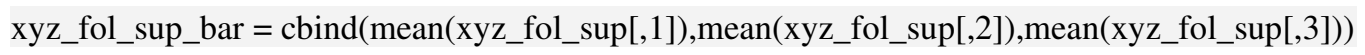

670

xyz_fol_prof_bar = cbind(mean(xyz_fol_prof[,1]),mean(xyz_fol_prof[,2]),mean(xyz_fol_prof[,3]))

671 S_fol_sup $=\operatorname{cov}\left(x y z \_f o l \_s u p\right) / n \_f o l \_s u p$

672

S_fol_prof $=\operatorname{cov}\left(x y z \_f o l \_p r o f\right) / n \_f o l \_p r o f$

673

mid_fol = S_fol_sup+S_fol_prof

674

mid_fol_inv = solve(mid_fol)

675

diff $=$ xyz_fol_sup_bar-xyz_fol_prof_bar

676

$\mathrm{T} 2 \_$fol $=$diff $\% * \%$ mid_fol_inv $\% * \% \mathrm{t}(\mathrm{diff})$ 

$\mathrm{v}=\left(\operatorname{tr}\left(\operatorname{mid} \_f o l \% * \% \mathrm{mid} \_f o l\right)+\left(\operatorname{tr}\left(\operatorname{mid} \_f o l\right)\right)^{\wedge} 2\right) /\left(\left(\operatorname{tr}\left(\mathrm{S} \_\right.\right.\right.$fol_sup $\% * \% S \_$fol_sup $\left.)+\left(\operatorname{tr}\left(\mathrm{S} \_f o l \_s u p\right)\right)^{\wedge} 2\right) /\left(\mathrm{n} \_\right.$fol_sup -1$\left.)\right) \quad+$ 679 ((tr(S_fol_prof\%*\%S_fol_prof)+(tr(S_fol_prof $\left.))^{\wedge} 2\right) /($ n_fol_prof-1)

680 \#fixando o quantil 0.95 da v.a. $\mathrm{T}^{\wedge} 2$ de Hotelling

$681 \mathrm{k}=$ qhotelling $(.95, \mathrm{v})$

682

T2_fol

683

684 diag(S_fol_sup)[1]/diag(S_fol_prof)[1]

685 diag(S_fol_sup)[2]/diag(S_fol_prof)[2]

686 diag(S_fol_sup)[3]/diag(S_fol_prof)[3]

687

688 \#install.packages("biotools")

689 \#Verificando que as variancias sao distintas

690 library("biotools")

691 biotools::boxM(dados[,6:8],factor(dados\$sup_prof))

692 biotools::boxM(dados_fol[,6:8],factor(dados_fol\$sup_prof))

693 biotools::boxM(dados_frat[,6:8],factor(dados_frat\$sup_prof))

694

695 \#testes (verificando as diferenças)

696 ICSNP::HotellingsT2(xyz_sup,xyz_prof, test = "chi")

697 ICSNP::HotellingsT2(xyz_frat_sup,xyz_frat_prof, test = "chi")

698 ICSNP::HotellingsT2(xyz_fol_sup,xyz_fol_prof, test = "chi")

699

700

701

702

703

704

705

706

707

708

709

710 


\section{References}

Anderson, M. J., Walsh, D.C.I. (2013) PERMANOVA, ANOSIM, and the Mantel test in the face of heterogeneous dispersions: What null hypothesis are you testing? Ecological Monographs, 83(4), pp. 557-574.

Ang, A.H.S., Tang, W. (2007) Probability Concepts in Engineering: Emphasis on Applications to Civil and Environmental Engineering. John Wiley \& Sons, New York, USA, 420 p.

Costa, E.A. (2005) Avaliação de ameaças e risco geotécnico aplicados à estabilidade de taludes. Dissertação de Mestrado, Universidade Federal do Rio Grande do Sul, Porto Alegre, RS, 160 p.

Fisher N. I., Lewis T., Embleton, B. J. J. (1993) Statistical analysis of spherical data. Cambridge University Press, New York, USA, $320 \mathrm{p}$.

Ge, H., Tu, J., Qin, F. (2011) Analysis of Slope Stability with First Order Second Moment Method. International Journal of Digital Content Technology and its Applications 5(12): 445-451.

Härdle, W. K. and Simar, L. (2012) Applied Multivariate Statistical Analysis. $3^{\circ}$ Ed., Springer Heidelberg Dordrecht London New York, USA 516 p.

Hasui, Y., Mioto J.A. (1992) Geologia Estrutural Aplicada, São Paulo, ABGE Associação Brasileira de Geologia de Engenharia e S.A. Indústrias Votorantim.

Hoek, E., Kaiser, P.K.,Bawden,,W.F. (1997) Support of Underground Excavations in Hard Rock. Ed. Balkema 215p.

Hudson, J.A. e Feng, X.T. (2015) Rock Engineering Risk. CRC Press 572 p.

Johnson, R.A., Wischern, D.W. (2007) Applied Multivariate Statistical Analysis, Pearson Prentice Hall, 6th edition p. 284-312.

Krueger, J. I., and Heck, P. R. (2019) Putting the p-value in its place. The American Statistician, 73 (sup1), $122-128$.

Mardia. K. V., (1972) Statistics of Directional Data. Academic Press, London.

Mardia, K.V., Kent, J. T., Bibby, J. M. (1995) Multivariate Analysis, Academic Press, $2^{\circ}$ edition.

Montoya, C.A.H. \& Assis, A.P. (2011b) Tools for reliability analysis in geotechnical engineering: application (in Spanish). Revista Ingenierías Universidad de Medellín, 10 (18): 79-86.

Pacheco, F.E.R.C., Serrano, M.S., Caxito, F.A., Moura, C.D., Quintão, D.A. (2017) Programa Mapeamento Geológico do Estado de Minas Gerais - Projeto Triângulo Mineiro: Folha de Uberlândia. Instituto de Geociências UFMG.

Pastore, E. L., Fontes, R. M., Oliveira, A. M. S., \& Brito, S. N. A. (1998) Geologia de Engenharia. Caracterização e Classificação de Solos Cap.12, 197-210.

Pimentel M.M., Fuck R.A., Botelho N.F., (1999) Granites and the geodynamic history of the Brasília Belt, central Brazil: a review. Lithos, 46:463-483.

Pimentel M.M. (2016) The tectonic evolution of the Neoproterozoic Brasília Belt, central Brazil: a geochronological and isotopic approach. Brazilian Journal of Geology, 2016, 46 (Suppl 1):67-82.

Sadowski, G.R. (2014) Previsibilidade e Risco em Geologia Geotécnica. In Direito da Construção, Org. Fernando Marcondes, Editora Pini. pp. 271-281. 
750 Strieder A.J. \& Nilson A.A. (1992) Mélange ofiolítica nos metassedimentos do Grupo Araxá de Abadiânia (GO) e 751 implicações tectônicas regionais. Revista Brasileira de Geociências, 22:204-215.

752 Valeriano, C. M., (1993) Evolução tectônica da extremidade meridional da faixa Brasília, região da represa de Furnas, 753 sudoeste de Minas Gerais. Tese de Doutorado, Instituto de Geociências, Universidade de São Paulo. São Paulo, 754 Doi: 10.11606/T.44.1993.tde-23062015-140732.

755

756

757

758 